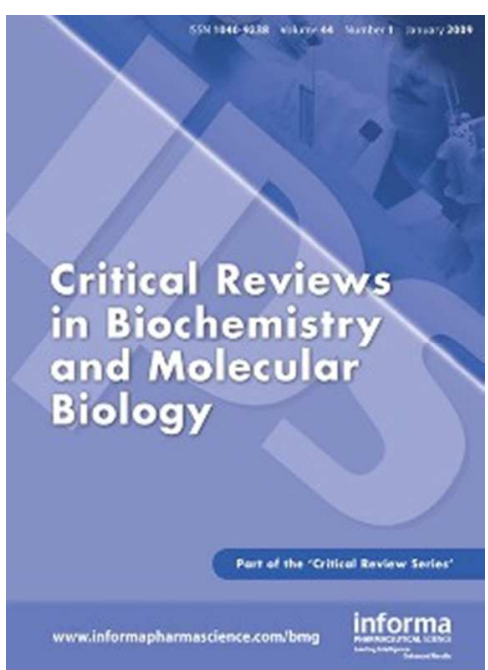

\title{
Structures of Ras Superfamily Effector Complexes: What have we learnt in two decades?
}

\begin{tabular}{|r|l|}
\hline Journal: & Critical Reviews In Biochemistry \& Molecular Biology \\
\hline Manuscript ID: & BBMG-2014-0032.R1 \\
\hline Manuscript Type: & Review \\
\hline Date Submitted by the Author: & n/a \\
\hline Complete List of Authors: & $\begin{array}{l}\text { Mott, Helen; University of Cambridge, Department of Biochemistry } \\
\text { Owen, Darerca; University of Cambridge, Department of Biochemistry }\end{array}$ \\
\hline Keywords: & Ras, Rho, Rab, Arf, Ran, small G protein, GTPase \\
\hline & \multicolumn{2}{|l}{} \\
\hline
\end{tabular}

\section{SCHOLARONE \\ Manuscripts}

URL: http:/mc.manuscriptcentral.com/bbmg Email: pfeffer@biochem.wisc.edu 


\section{Structures of Ras Superfamily Effector Complexes: What have we learnt} in two decades?

Helen R. Mott \& Darerca Owen

Department of Biochemistry, University of Cambridge. 80, Tennis Court Road, Cambridge CB2 1GA. UK.

Keywords:

Ras, Rho, Rab, Arf, Ran, GTPase, small G protein 


\begin{abstract}
The Ras superfamily small $\mathrm{G}$ proteins are master regulators of a diverse range of cellular processes and act via downstream effector molecules. The first structure of a small G protein-effector complex, that of Rap1A with c-Raf1, was published 20 years ago. Since then, the structures of more than 60 small $\mathrm{G}$ proteins in complex with their effectors have been published. These effectors utilize a diverse array of structural motifs to interact with the $\mathrm{G}$ protein fold, which we have divided into four structural classes: intermolecular $\beta$-sheets, helical pairs, other interactions and $\mathrm{PH}$ domains. These classes and their representative structures are discussed and a contact analysis of the interactions is presented, which highlights the common effector-binding regions between and within the small $\mathrm{G}$ protein families.
\end{abstract}


1. Introduction

Small GTPases of the Ras superfamily are a large group of proteins that are related by a single, overriding property: their ability to bind GDP or GTP. The sequence features that are required for nucleotide binding and, in many cases, intrinsic hydrolysis of bound GTP, are conserved between the diverse members of the superfamily. These features lead to an overall conserved fold (the G domain) that is exemplified by HRas (Figure 1.1), the first small G protein whose structure was solved (Pai et al. 1990, Milburn et al. 1990). Comparison of the structures of H-Ras in its GDP-bound and GTP-bound forms allowed the definition of two regions of the protein that are exquisitely sensitive to the bound nucleotide. These regions are known as switch 1 and switch 2 and in H-Ras encompass residues 28-40 and 58-70 respectively. The switch regions mediate conformational changes in response to the change in nucleotide (reviewed in Wittinghofer and Vetter 2011). There are two invariant residues, Thr35 in switch 1 and Gly60 in switch 2 (Ras numbering), whose mainchain NH groups form hydrogen bonds with the terminal phosphate group of GTP. These interactions are responsible for dragging the rest of the switch residues into new conformations, where they are poised to make interactions with other molecules. This mechanism has been dubbed the 'loaded spring', which emphasizes that the GTPbound form is the more rigid state of the enzyme, whereas the GDP-bound form can be thought of as the relaxed conformation (Vetter \& Wittinghofer 2001).

The Ras superfamily has been divided into five families based on their sequence and functional differences (reviewed in (Takai et al. 2001)). The family members all contain a $\mathrm{G}$ domain fold, which is embellished by extra features in the Rho, Arf and Ran families (Figure 1.2). The superfamily roster comprises 167 proteins in humans, of which there are 39 in the Ras family, 22 Rho proteins, 65 Rabs, 22 Arfs, 1 Ran and 10 unclassified sequences (Rojas et al. 2012). The Ras family is involved in controlling cellular proliferation, protection from apoptosis and cell differentiation and includes the three Ras isoforms (H-, K- and N-Ras) as well as the Rap and Ral proteins. The Rho family regulates actin dynamics and in doing so impinges on several essential processes, such as cell division, cell migration and vesicle transport. Its members include RhoA-C, Rac1-3 and Cdc42. The Rab and Arf families together control the complex interplay of vesicle trafficking necessary for the proper functioning of a eukaryotic cell (reviewed in (Mizuno-Yamasaki et al. 2012)). The 
complexity of these processes accounts for the large size of the Rab subfamily, which is responsible for the vectorial nature of vesicle traffic. The smallest family is the Ran family, which contains a single member but being responsible for nuclear transport it is also one of the most abundant small G proteins in the cell.

The Ras superfamily proteins can exist in two nucleotide-bound forms and thus most are considered to behave as a cellular switch. In general, the proteins are not responsible for switching themselves but rely on auxiliary proteins that help to switch them on, switch them off or maintain them in the off state (reviewed in (Cherfils \& Zeghouf 2013)). The large and diverse group of guanine-nucleotide exchange factor (GEF) proteins is responsible for switching on small $G$ proteins. They usually achieve this by reducing their affinity for GDP and stabilizing the nucleotide-free (or apo) form. This allows GTP (which is more abundant in cells) to take its place and leads to the active $\mathrm{G}$ protein conformation being formed. The GTP, when bound, can be slowly hydrolysed to GDP by most G proteins, but the intrinsic rate of hydrolysis is rather too slow to be useful for control of cellular signaling. The hydrolysis rate is stimulated by the GTPase activating proteins (GAPs), another large and diverse group of proteins, which are under various forms of regulation.

Ras superfamily proteins of the Ras, Rho and Rab families are modified at cysteine residues in their C-terminus with farnesyl, geranylgeranyl and palmitoyl moieties. These acylations localize the small $\mathrm{G}$ proteins to cellular membranes, which is where their signaling activities take place. In contrast, the Arf family generally have a myristoyl group added to their N-terminus, while the Ran proteins are not modified by lipidation at all. The Rho and Rab families can be stabilized in their GDP-bound form in the cytosol by their interaction with the guanine nucleotide dissociation inhibitors (GDIs). The GDI proteins bind to both the switch regions and to the hydrocarbon chain of the isoprenyl group and thus allow removal of these $G$ proteins from their membranes (Cherfils \& Zeghouf 2013). This is essential for the function of Rabs, which need to be recycled back to their original donor membrane compartment. For the Rho family proteins, it presumably allows for a greater level of control: their signaling will be prevented by their removal from the membrane and they can be shuttled between different internal membranes and the plasma membrane. 
The diverse cellular roles performed by small G proteins are mediated by the effector proteins. These molecules bind specifically to the active, GTP-bound form of small G proteins and are responsible for propagating signals to downstream pathways. Given that there are almost 160 known small $\mathrm{G}$ proteins and each one can bind several effector proteins, it is apparent that there are a bewilderingly large number of these pathways.

The first structure of a small $G$ protein with its downstream effector was solved 20 years ago by the Wittinghofer group (Nassar et al. 1995) and included the Rasbinding domain from c-Raf in complex with Rapla bound to a GTP-analogue. Since this milestone, more than 60 structures of $\mathrm{G}$ protein-effector complexes have been solved. Here we will review the main features of these structures, considering only those complexes whose structures have been deposited in the protein data bank (PDB). They have been divided into structural classes rather than by the function of the $G$ proteins or their effector molecules: a number of effectors bind using an intermolecular $\beta$-sheet that forms to extend the antiparallel $\beta 2-\beta 3$ strands of the $G$ domain; the second structural class utilize a pair of $\alpha$-helices that contact the switch regions of the $\mathrm{G}$ domain; the third class includes the structures that do not use either of these structural motifs to bind to the G domain and the fourth class include those effectors that use a PH domain. The small G protein families and their effector complexes are summarized in Table 1, along with their PDB identifiers. In the cases where there is more than one structure of the same complex, we have used the highest resolution structure for our analysis. 


\section{Intermolecular $\beta$-sheet complexes}

\subsection{The Ras family}

\subsubsection{Raf effector complexes}

The long awaited first atomic resolution structure of a small $\mathrm{G}$ protein-effector complex was finally published in 1995 (Nassar et al. 1995), six years after the initial structures of Ras (Pai et al. 1989, de Vos et al. 1988) and two years after the discovery of Raf, the first bone fide effector protein identified for Ras ((Vojtek et al. 1993). It was not, however, a Ras-Raf complex that was revealed but rather Rap1A with Raf RBD. Rap1A, a close relative of Ras is 50\% identical to Ras overall but has $100 \%$ identity across the effector binding region and indeed shares all the known Ras effectors. The crystal structure of Rap1A-Raf RBD showed the formation of an elegant intermolecular $\beta$-sheet formed between two anti-parallel $\beta$-strands: $\beta 2$ from Rap1A and B2 from the Raf RBD. The two proteins came together in a seamless merger and heralded a theme for small $G$ protein-effector complexes that still persists. Subsequent structures of mutant variants of Rap1A showed molecules predicted to be ever closer in structure to Ras itself in complex with Raf (Nassar et al. 1995). In fact it was not until 2013 that the structure of H-Ras-Raf RBD was finally available (PDB code: $4 \mathrm{GON})$.

The structures demonstrate that the Ras-Raf interface comprises primarily the intermolecular $\beta$-sheet but also involves contacts between the C-terminal end of Raf helix A1 and Ras/Rap1A (Figure 2.1A,B). The buried surface area is relatively modest at $\sim 1,200 \AA^{2}$. Although not now a surprise, originally the structure was striking for the lack of contacts between the effector and the $\gamma$-phosphate of GTP on the G protein, while association with what was already described as the 'effector binding loop' (residues 32-40) of Rap1A was less unexpected. The interaction is mediated by a comprehensive mesh of mainly polar interactions from both mainchain and sidechains groups from charged residues across the interface, with only a few hydrophobic contributions. Contacts on Ras involve Ile21, Gln25, Val29, Glu31, Asp33, Ile36, Glu37, Asp38, Ser39, Tyr40 and Arg41 with strong salt bridges forming between Glu31 ${ }^{\text {Ras }}$-Lys84 ${ }^{\text {Raf }}$, Glu3 $7^{\text {Ras }}$-Arg59 $9^{\text {Raf }}$ Glu37 ${ }^{\text {Ras }}-\operatorname{Arg} 67^{\text {Raf }}$ and 
Asp38 ${ }^{\text {Ras }}-\operatorname{Arg} 89^{\text {Raf. }}$. A series of strong hydrogen bonds also exist between Glu37 $7^{\text {Ras }}$ Val69 ${ }^{\text {Raf }}$, Asp3 $38^{\text {Ras }}-$ Thr6 $68^{\text {Raf }}$, Ser39 ${ }^{\text {Ras }}-$ Arg67 $7^{\text {Raf }}$, Ser38 ${ }^{\text {Ras }}-$ Arg89 $9^{\text {Raf }}$, Ser39 $9^{\text {Ras }}-\operatorname{Arg} 67^{\text {Raf }}$ and Arg $41^{\text {Ras }}-\mathrm{Gln} 66^{\text {Raf }}$. Interestingly, important contributions come from residues outside the intermolecular $\beta$-sheet with salt bridges seen between Glu31 ${ }^{\text {Ras }}$-Lys $84^{\text {Raf }}$ and Asp33 ${ }^{\text {Ras }}$-Lys $84^{\text {Raf }}$. Specificity for the interaction also lies in these regions of the protein with Glu3 $1^{\text {Ras }}$ being substituted for Lys $31^{\text {Rap1A }}$ in Rap1A explaining the decreased affinity of Rap1A for $\operatorname{Raf}(1.2 \mu \mathrm{M})$ in comparison to Ras (18nM) (Nassar et al. 1996). These initial structures also revealed the Raf RBD to be a ubiquitin fold domain as both previously predicted (Emerson et al. 1994) and demonstrated (Emerson et al. 1995), defining the first small G protein binding module. The Raf RBD shows little structural change between the free and bound forms.

\subsubsection{Ras-RalGDS}

The initial Rap1A-Raf RBD structure turned out to be the first in a rather slow progression of structures of Ras-effector complexes. The next complex structure to be reported was that of Ras-RalGDS in 1998 (Huang et al. 1998). Whereas Raf binds tightly to Ras and Rap1A binds with a weaker affinity, requiring the use of Rap1A mutants to achieve tight complexes, the specificity is reversed with RalGDS, with Ras binding relatively weakly compared with Rap1A (Herrmann et al. 1996). Therefore this first structure of Ras in complex with one of its effectors again necessitated the use of mutants with Ras E31K crystallized with the RalGDS RBD, the mutation making Ras, in this instance, more similar to Rap1A. The Raf RBD and RalGDS RBD only display $13 \%$ sequence identity and yet the structure showed that the RalGDS RBD assumed the same ubiquitin-like fold as the Raf RBD. In fact the complex showed the proteins forming a very similar intermolecular $\beta$-sheet to Rap1A-Raf RBD with the major interaction occurring between two anti-parallel $\beta$ strands; $\beta 2$ of Ras and B2 in RalGDS. The buried surface area was also similar to the Rap1A-Raf complex at $1,150 \AA^{2}$. The RalGDS RBD only shows small local changes between the free and bound forms (Huang et al. 1998, Vetter et al. 1999a). When the complexes are superimposed using the small $\mathrm{G}$ proteins as the anchor, the effector domains are seen to rotate by about $35^{\circ}$ with respect to each other. The two juxtaposed strands from the respective partners in the complex are therefore tilted in 
the Ras-RalGDS complex but notwithstanding this, the mainchain interactions within the two complexes are similar (Figure 2.1B,C).

Mutational analysis between the Raf and RalGDS complexes had indicated that the energetics of the binding surfaces on the $G$ protein would be significantly different in the two complexes. Major contacts on Ras for RalGDS again mainly involve switch 1 and include residues, Lys31, Asp33, Pro34, Thr35, Ile36, Glu37, Asp38, and Tyr40. The interactions are largely salt bridges and hydrogen bonds with a few hydrophobic contacts. Salt bridges are formed between Lys31 ${ }^{\text {Ras }}$-Asp56 ${ }^{\text {RalGDS }}$, Asp33 ${ }^{\text {Ras }}$ Lys $52^{\text {RalGDS }}$ and Glu37 $7^{\text {Ras }}$-Arg20 RalGDS . The hydrogen bond network consists of bonds between Lys31 ${ }^{\text {Ras }}$-Asp51 ${ }^{\text {RalGDS, }}$ Lys31 ${ }^{\text {Ras }}$-Asn54 ${ }^{\text {RalGDS }}$, Pro34 ${ }^{\text {Ras }}$-Lys52 ${ }^{\text {RalGDS }}$, Thr35 ${ }^{\text {Ras }}$ Lys52 ${ }^{\text {RalGDS }}$ Asp38 ${ }^{\text {Ras }}$-Lys52 ${ }^{\text {RalGDS }}$, Tyr40 ${ }^{\text {Ras }}$-Lys $32^{\text {RalGDS }}$ and Glu37 ${ }^{\text {Ras }}-$ Tyr31 ${ }^{\text {RalGDS }}$. While both Raf and RalGDS share common binding residues on Ras, differences are seen with Glu31, Ser39 and Arg41. The two structures together explain some of the mutation data available. E37G Ras was known to bind to RalGDS but not to Raf (Rodriguez-Viciana et al. 1997, White 1995). Glu37 is quite differently orientated in the two complexes, facing the interface in the Raf complex but orientated away from the interface in the RalGDS complex, hence its importance to Raf but not RalGDS binding. T35S was known to abrogate the interaction with RalGDS while retaining the ability to interact with Raf. The effects of this mutation are not so clear from the structures. Thr35 is not involved in contacts in the Raf structure, so the lack of effects of the T35S mutation is not surprising. In the RalGDS structure however Thr35 makes a H-bond with Lys51 through a water molecule. It is likely that this interaction could be maintained in a substitution with serine. A more likely explanation of this mutation is that T35S changes the dynamics of switch 1 in Ras, which then affects RalGDS binding.

\subsubsection{Ras-PI3 Kinase}

The structure of Ras in complex with its third major effector, PI3K, was published in 2000 (Pacold et al. 2000). The complex consists of H-Ras bound to PI3K $\gamma$ V223K. The V223K mutation increases the affinity of PI3K $\gamma$ for Ras and facilitated crystallization. This structure contained the first full effector protein in contrast to the previously described RBDs and therefore promised details of the mechanism of 
activation of the effector. The structure again described the formation of an intermolecular $\beta$-sheet between the PI3K RBD and Ras, with the RBD adopting the now omnipresent ubiquitin-fold (Figure 2.1D). Again, at the interface, strand $\beta 2$ of Ras paired with strand B2 of the PI3K RBD. However this new structure revealed a unique feature: in this case the PI3K RBD interacted not only with switch 1 of Ras but also with switch 2, the second nucleotide sensitive region of the G protein. Until now, switch 2 binding had been the preserve of regulators like the GAPs and GEFs. On binding to Ras, loop 3 of the PI3K RBD becomes ordered and prevents Ras binding in the same orientation as with either Raf or RalGDS. Under these conditions new contacts are made between Ras switch 2 and both the PI3K RBD and the catalytic domain.

The contacts made by switch 1 of Ras in the PI3K complex again consist of the network of salt bridges and hydrogen bonds. Ras residues involved in PI3K contacts in switch 1 comprise Asp33, Ile36, Glu37, Asp38, Ser39, Tyr40 and Arg41, while new contacts in switch 2 involve Glu63, Tyr64 and Arg73. The overall buried surface area however remained in line with the previous two complexes at $\sim 1,300 \AA^{2}$.

The three structures together show that although Ras uses a common mode of interaction with its effectors, it may discriminate between them due to a rotation with respect to each of the effectors. The $\beta$-strand that forms the intermolecular $\beta$-sheet is in a slightly different orientation in each of the different effectors when the structures are overlaid on HRas (Figure 2.1B,C,D).

Most work with Ras mutants activating PI3K had been performed using PI3K $\alpha$ : differences between PI3K $\alpha$ and PI3K $\gamma$ gave rise to different effects with the Ras mutations but most can be explained in light of this structure. Selective abrogation for PI3K by Ras Y64G was relatively easy to interpret, as only the PI3K complex shows switch 2 contacts. The effect of the classic mutation of Ras Y40C, which retains the ability to bind PI3K while abrogating binding to both Raf and RalGDS required some speculation. Y40C attenuates binding to PI3K $\gamma$ probably due to a hydrogen bond forming between Tyr40 ${ }^{\text {Ras }}$ and Gln231 ${ }^{\mathrm{P} 3 \mathrm{~K}}$. Gln231 however is not conserved in PI3K $\alpha$ allowing the mutation to be tolerated. The structure also showed how the 
$\mathrm{V} 223 \mathrm{~K}^{\mathrm{PI} 3 \mathrm{~K}}$ mutation introduced to facilitate tighter binding mediates its effects: Lys223 forms an additional hydrogen bond with Glu37 in Ras switch 1. By comparison of PI3K $\gamma$ in the free and Ras-bound forms it was apparent that a number of structural changes are seen in the catalytic domain, suggesting a mechanism of activation by Ras. Full details of the mechanism however await structural information on substrate binding.

\subsubsection{Ras-Byr2}

Interestingly the next structure to emerge was that formed between Ras and the $S$. pombe Raf homologue, Byr2. Again the same story unfolded with the Byr2 RBD adopting a ubiquitin-fold which then formed an intermolecular $\beta$-sheet with Ras, with the contact edges compromising $\beta 2$ Ras and B2 Byr2 and mediated by a network of polar interactions. Again $\sim 1,200 \AA^{2}$ of accessible surface area was buried in the interaction. Although very similar to the structures already presented, this structure confirmed that the mechanism of communication used by Ras to talk to its effector proteins was conserved right down to the lower eukaryotes (Scheffzek et al. 2001).

\subsubsection{Ras- PLC $\varepsilon$}

PLC $\quad \square \square \square \square \square \square$ relatively $\square \square \square \square \square \square \square \square \square \square \square \square \square \square \square$ he $\square \square \square \square \square$ effector family

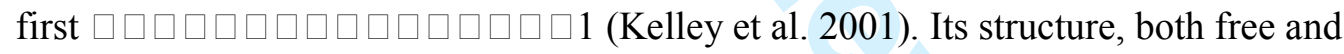
in complex with Ras, followed in 2006 (Bunney et al. 2006).

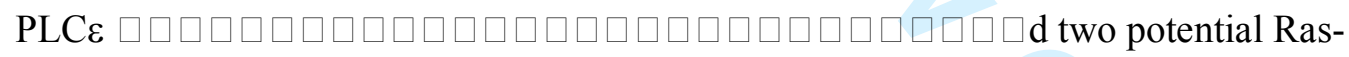
association (RA) domains (as the ubiquitin-like domain had now become known):

RA1 and RA2, of which only RA2 had been demonstrated to bind H-Ras in a classic effector manner (i.e. with GTP dependence) and with high affinity (Kelley et al. 2001). Both RA domains were shown to adopt remarkably similar ubiquitin-like folds, however an examination of the surface charge distribution showed that RA1 lacked the abundance of positively charged sidechains facing the small G protein, found in most RA domains examined by this point (Bunney et al. 2006). Overall the Ras-PLC $\varepsilon$ RA2 complex was structurally similar to the previous Ras-effector complexes. In detail, this complex was more similar to the Ras-PI3K structure, with the effector contacting switches 1 and 2. Contacts on Ras involved Gln25, Glu31, Ile36, Glu37, Asp38, Ser39, Arg41, Glu63 and Tyr64, a very similar profile to the 
interacting Ras residues in the PI3K structure. Like the other effectors, the RA2 structure does not change significantly when complexed to Ras.

The structure again allowed an analysis of the mechanism behind the effects of the discriminatory mutations used widely in the field. Previous studies had warned that Ras E37G, used to activate RalGDS uniquely, also activated PLCE (Kelley et al. 2001) and this was confirmed by the PLCe structure. The Ras D38N mutant had previously been used as a universal inhibitor but was now shown to be capable of activating PLCE (Kelley et al. 2001). Ras D38N binding was shown to be compatible with complex formation in the structure, however it was also demonstrated that indeed this mutation could also bind to PI3K at elevated concentrations. As more structures were solved the nuances of the signalling networks were becoming more apparent, revealing cautionary advice to the use of inhibitors and activators in vivo.

\subsubsection{Ras-NORE1}

So far all the effector proteins for Ras proteins had been enzymes of some description. In contrast to this, a new class of effectors had been identified in 2000, which had rapidly expanded to be a new family of 13 adaptor effectors, the RASSFs. The founding member, RASSF5A (originally named NORE1A) had a classic RA but surprisingly it was insufficient alone to bind Ras. A construct extended 68 residues at the N-terminus however was capable of binding H-Ras (Stieglitz et al. 2008). Successful crystallization of the complex required the use of 3 mutations: 2 in H-Ras, the classic D30E/E31K Rap1A mimics and K302D in NORE1A, which had previously been demonstrated to reduce specificity in NORE1A for Ras-Rap1A. NORE1A was revealed to have a classic ubiquitin-like folded RA domain, however it was unique in displaying an insertion between $\beta 1$ and $\beta 2$ and an $\mathrm{N}$-terminal extension as binding studies had predicted. The whole binding module comprised a 5stranded $\beta$-sheet with 2 flanking helices and one additional 310 -helix, forming a ubiquitin $\alpha-\beta$ roll. The $N$-terminal extension comprised a helix $\alpha_{N}$ and a short strand $\beta_{\mathrm{N}}$, connected by a type 1 reverse turn. This extension packed back tightly onto the ubiquitin-fold predominately through hydrophobic interactions between $\beta 1$ and $\alpha 2$, making it a unique binding module for the Ras family (Figure 2.1E). The classic intermolecular $\beta$-sheet still formed between Ras Switch 1/ $\beta 2$ and B2 of NORE1A, 
however the contacts were more extensive than in other complexes involving 10 residues of Ras switch 1 and 5 residues of NORE1A B2. The reverse turn in NORE1A formed hydrophobic interactions with Met67 and Tyr64 of Ras switch 2, making NORE1A another example of the then minority effectors that utilize interactions with switch 2 of Ras. In line with the extended RA in NORE1A the buried surface area in the complex is slightly greater than others at 1,546 $\AA^{2}$. Interestingly a prolonged lifetime was reported for the Ras-NORE1A complex, with a distinct contribution from the switch 2 interaction. These distinctive kinetics may be useful for a GTPase-adapter complex (Stieglitz et al. 2008).

\subsubsection{Ras-Grb14}

Another example of an adapter effector for Ras completes the picture of structures we have available for Ras proteins. The year 2013 saw the publication of Ras-Grb14 (Qamra \& Hubbard 2013). Grb14 is a member of the Grb7-10-14 family of cytoplasmic adapter proteins. Grb14 has a central RA domain followed by a PH domain and the RA and PH domains together are required for negative regulation of the insulin receptor by Grb14. It had already been observed with the RA-PH didomains of RIAM and Grb10 that the two domains and their linker pack together intimately to create effectively a single domain (Depetris et al. 2009, Wynne et al. 2012). Despite extensive contacts between the RA-PH domains, in each structure, the B2 strand of the RA was available to contact a small GTPase in the expected manner. The Ras-Grb14 RA-PH structure showed an exclusive interaction between the Grb14 RA and Ras, engagement following the general rules expected. A small hydrophobic cluster of interactions was seen between Ras residues both in switch 1 (Ile36) and also in switch 2 (Tyr64 and Met67), making engagement of switch 2 no longer a minority activity.

\subsubsection{Rap1A-KRIT1}

Not forgetting that Rap1A is a biological player in its own right and not just a convenient substitute for Ras, structures continued to emerge for Rap1A and in 2012 a novel twist on the ubiquitin-fold interaction emerged. KRIT1 is an effector protein for Rap1A with a 10-fold higher affinity for Rap1A over Ras. KRIT1 contains 4 ankyrin repeats followed by a FERM domain and binds to Rapla using the latter (Glading et al. 2007). The KRIT1 FERM domain comprises 3 lobes, F1, F2 and F3. 
The structure of the Rap1A-KRIT1 FERM structure shows extensive contacts between Rap1A switches 1 and 2 and FERM lobes F1 and F2 burying 1,750 $\AA^{2}$ of accessible surface area (Li et al. 2012). The F1 lobe of FERM domains was already known to adopt a ubiquitin-like fold, so it came as no surprise to see the intermolecular $\beta$-sheet form between Rap1A $\beta 2$ and B2 of the FERM F1 (Figure 2.1F). The FERM F2 lobe forms an acyl-CoA-binding protein-like fold and this was the first demonstration of such a class of domains interacting with a small $\mathrm{G}$ protein. The binding site on Rap1A starts with Gln25, which interacts with both Leu529 and Arg452 on KRIT1; this is followed by a hydrophobic interaction between Ile27 Rap1A with Pro525 KRIT , the KRIT1 residues lying in the F2 lobe. The intermolecular $\beta$-sheet region involves interactions including now-expected participation by Pro34, Thr35, Glu37, Asp38, Ser39 and Tyr40 of $\beta 2 /$ switch 1 on Rap1A: all interact with residues from the expected F1 lobe of the KRIT1 FERM. Switch 2 of Rap1A also interacts with residues from the F1 lobe, interactions being observed between Met67 $7^{\text {Rap1A }}$ and

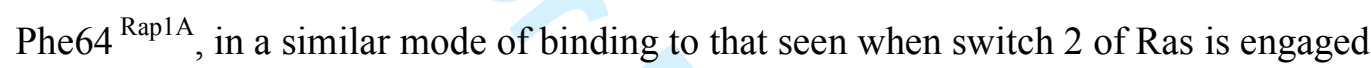
by effectors. Finally, extra contacts are observed between Gln $43^{\text {Rap1A }}$, Glu45 Rapla and Gln52 $2^{\text {Rap1A }}$ with F2 residues from KRIT1. This new contact site involves the Cterminus of $\beta 2$ and the $\mathrm{N}$-terminus of $\beta 3$ of Rap1A. A second report on the ternary structure that forms between Rap1A-KRIT1 and HEG1 shows some differences with the Rap1A-KRIT1 complex (Gingras et al. 2013). In the ternary structure Rap1A switch 2 is mobile and does not appear to make contacts with the F1 lobe of KRIT1. This is supported by mutagenesis data indicating that introduction of M67A or F64A makes little difference to complex formation. However there are differences in the proteins used in the two structures. Rap1A G12V-KRIT Y419F formed the binary complex, whereas Rap1A wt-KRIT wt participated in the ternary complex. Both structures recognize the importance of Rap1A Glu45 in driving the specificity of KRIT1 for Rap1A over Ras. FERM domains are widely spread amongst protein families including signaling kinases and phosphatases. Whether this turns out to be a new small $\mathrm{G}$ protein engagement module remains to be seen.

\subsubsection{Rap1A-RIAM}

The final structure we have at our disposal involving Ras-Rap1A shows us the complex that forms between Rap1A and the RA-PH didomain of RIAM (Zhang, 
Chang, et al. 2014a). RIAM is the adapter molecule that facilitates Rap1A control of integrin activation via inside-out signaling. RIAM binds to Rap1A specifically and shows little involvement in Ras-controlled signaling pathways. The Rap1A-RIAM structure therefore provides interesting insights into specificity mechanisms. Only a G12V/Q63L Rap1A construct yielded crystals when co-crystalized with the RIAM RA-PH construct. Mimicking the Ras-Grb14 structure, the Rap1A-RIAM structure shows that Rap1A binds exclusively to the RA domain of RIAM forming the canonical intermolecular $\beta$-sheet via $\beta 2$ Rap1A and B2 RIAM RA. The interaction exclusively involves switch 1 of Rap1A, with hydrogen bonds formed by Gln25, Lys31, Asp33, Glu37, Ser39 and Tyr40. A water-mediated network of hydrogen bonds further stabilizes the interaction surface, which is relatively modest in terms of buried surface area; in fact the complex shows the lowest Ras/Rap1A effector complex interface surface. Specificity of the interaction seems to lie largely in the Lys $31^{\text {Rap1a }}-$ Glu212 ${ }^{\text {RIAM }}$ salt-bridge. The importance of Lys31 in Rap1A specificity has been seen in many of the complexes described and also seems crucial for Rap1ARalGDS association. The RIAM RA-PH shows no significant changes between its free and bound forms.

\subsubsection{Emerging trends}

Overall the structures we have available point to a number of trends. The use of a ubiquitin-like fold as a binding module for Ras is the most obvious, however it is not the fold itself that determines binding but rather the charge distribution on the surface. In general Ras is mainly negatively charged and its effector proteins have a complementary positively charged binding interface (Figure 2.2). Where data are available, interaction between Ras and its effectors appear to be driven by high association rates coupled to high dissociation rates (Gorman et al. 1996, Sydor et al. 1998, Linnemann et al. 1999, Linnemann et al. 2002). Affinity seems to be dictated mainly by changes in the dissociation rate, with association rates being similar between complexes (Linnemann et al. 2002). Interestingly the lifetime of the Ras complexes are shorter than the expected lifetime of GTP intrinsic hydrolysis. The rapid cycling of these high affinity effector complexes would therefore allow Ras not only to activate multiple effectors but also be available for downregulation by GAP proteins. 


\subsubsection{RalA Complexes}

While the Ras and Rap isoforms are the most closely related members of the Ras family, structures of other members in complex with their effector proteins are also available. The two Ral isoforms, RalA and RalB share $82 \%$ identity with each other and 55\% identity with Ras. In 2003 and 2005 structures of RalA in complex with the RBDs of Sec5 and Exo84 were published (Fukai et al. 2003, Jin et al. 2005). Sec5 and Exo84 are two components of the exocyst complex, which regulates exocytosis, and both are Ral effector proteins. The RBD of Sec 5 had already been shown to be an Iglike domain (Mott et al. 2003), which, as an all $\beta$-strand domain, was a prime candidate to be another intermolecular anti-parallel $\beta$-sheet effector interaction. The RalA-Sec5 complex confirmed this, showing the classic complex conformation with the interface comprising $\beta 2$ RalA and $\beta 1$ Sec5 (Fukai et al. 2003) (Figure 2.3A). The interface shows a typical network of hydrogen bonds and contact residues on RalA included Tyr36, Glu38, Lys47, Ala48, Ser50 and Arg52 (for Ras numbering subtract 11). The intermolecular sheet formed between RalA-Sec5 was most similar to the Ras-PI3K interface. Again no substantial changes are seen between the free and bound forms of the effector RBD. The buried surface area of the complex was $\sim 1,000$ $\AA^{2}$.

The RalA-Exo84 structure followed in 2005. Here, the Exo84 RBD was revealed to be another multi-functional domain, a PH domain. Although best known for their ability to recruit proteins to membranes by binding phosphoinositides, $\mathrm{PH}$ domains also function as protein-protein interaction modules. Unlike Sec5, Exo84 interacts with both switch 1 and 2 on RalA (Figure 2.3B), together with residues outside these regions, and buries a significantly larger surface area at $1,700 \AA^{2}$. Interacting residues on RalA include: Lys47, Ala48 and Ser50 in Switch 1; Glu73, Tyr75, Asn81 and Tyr82 in Switch 2; Lys16 and Arg52. Interestingly, despite the formation of the intermolecular $\beta$-sheet in the complex, this structure was the first demonstration of a parallel intermolecular $\beta$-sheet being formed in a Ras family small G protein-effector complex. 
The binding sites for the two effectors on RalA are partially overlapping, sharing RalA residues; Ala48, Ser50 and Arg52. In fact mutations at these residues seem to mimic the partial loss of function mutations used so widely with Ras: A48W and S50W both abrogate Sec5 binding while having little effect on Exo84. Conversely R52W retains Sec5 binding but prevents the interaction with Exo84.

So the two structures of the Ral-effector complexes followed the same intermolecular $\beta$-sheet theme (albeit with subtle differences in Exo84) but introduced two new recognition domains in the effector proteins, an Ig-like domain and a $\mathrm{PH}$ domain.

\subsection{The Rho family}

As soon as the Rho family members were discovered it was clear that they would look slightly different to the Ras proteins. The Rho family small $\mathrm{G}$ proteins adopt the canonical Rossman fold of all G proteins but sequence alignments showed that they all have $\sim 10-15$ extra amino acids. Once the first Rho family structures were solved it was found that these extra amino acids of the 'insert loop', form a pair of $\alpha$ helices that are missing in Ras (Figure 1.2). This is the defining structural characteristic of the Rho proteins. Despite work from many groups, nothing has been shown to interact directly with this insert region although early functional assays suggest that it is necessary for transformation, indicating that it has a crucial role.

\subsubsection{Cdc42-specific CRIB effectors}

Structures of the Rho family small G proteins in complex with their effectors did not lag far behind the Ras family. The first structures were published in 1999 and showed the Rho family protein Cdc42 in complex with two effectors from the CRIB (Cdc42, Rac interactive binding) family, ACK and WASP (Mott et al. 1999; Abdul-Manan et al. 1999). The GBDs in these two proteins were distinctly different to the Raf RBD (the only effector complex structure published prior to these). While the Raf RBD was a preformed, structured domain, the CRIB regions of both ACK and WASP were disordered in their free forms. However, on binding to $\mathrm{Cdc} 42$, both interaction domains adopted discrete structures to display the same anti-parallel intermolecular $\beta$ sheet seen in Ras-Raf (Figure 2.4A,B)

URL: http:/mc.manuscriptcentral.com/168mg Email: pfeffer@biochem.wisc.edu 
The complexes however looked completely different to the Ras-Raf structure. WASP adopted an extended conformation, the $\mathrm{N}$-terminal portion of which (containing the CRIB motif) contacts switch $1, \beta 2$ and $\alpha 5$ of $\mathrm{Cdc} 42$ adding an irregular $\beta$-strand to form the intermolecular $\beta$-sheet. The $\mathrm{C}$-terminal portion then formed a $\beta$-hairpin followed by an $\alpha$-helix, which packed against Cdc42 switch 2. This extended conformation of WASP resulted in an extensive interface which buries $\sim 2,900 \AA^{2}$ of accessible surface area. Not surprisingly an increased number of $\mathrm{Cdc} 42$ residues are involved including Ile21, Thr25, Val36, Phe37, Asp38, Asn39, Tyr40, Ala41, Ile46, Gly47, Tyr51, Phe56, Leu67, Leu70, Glu171, Ile173, Leu174 and Leu177. The ACK GBD also forms an extended conformation, which wraps around $\mathrm{Cdc} 42$ burying $\sim 4,200 \AA^{2}$ of accessible surface area and producing the largest interface of an intermolecular $\beta$-sheet type interaction. Contacts on Cdc42 include Leu20, Ile21, Asp38, Ala41, Val42, Thr43, Val44, Met45, Ile46, Leu67, Leu70, Lys166, Leu174 and Leu177, a profile not dissimilar to the contacts made by WASP. The N-terminus of the GBD interacts with Cdc42 $\alpha 5$, particularly using hydrophobic contacts e.g. Leu174 ${ }^{\mathrm{Cdc} 42}$-Leu $449^{\mathrm{ACK}}$. The GBD then adopts a $\beta$-strand that forms an anti-parallel intermolecular $\beta$-sheet with $\mathrm{Cdc} 42$ followed by extensive contacts with switch 1 . The ACK GBD forms a hairpin but no other regions of secondary structure. As usual the structures explain many mutations known to affect these interactions. Asp38 mutations were known to be deleterious to the binding of most CRIB family effectors and this residue is seen contacting the two conserved histidine residues of the CRIB motif in both structures.

Despite the retention of the intermolecular $\beta$-sheet in these complexes, the extended conformation of the binding regions was very different to the Ras family structures. The fine details were also different as, in contrast to the Ras family complexes, these two structures show extensive hydrophobic interactions.

\subsubsection{PAK effector complexes}

The structure of Cdc42 in complex with a third CRIB effector, PAK, was published in 2000) (Morreale et al. 2000). The free form of the PAK GBD indicated that a short $\alpha-$ helix was present, in contrast to the disordered free forms of ACK and WASP. The 
CRIB region of PAK formed an intermolecular $\beta$-sheet with $\mathrm{Cdc} 42$, utilizing residues 75-83 of PAK and 40-46 of Cdc42 (figure 2.4C). As was observed in the previous two CRIB complexes, hydrophobic residues at the N-terminus of PAK (Ile75 and Leu77) make contacts with $\alpha 5$ on $\mathrm{Cdc} 42$. The $\mathrm{C}$-terminal region of PAK adopts a $\beta$-hairpin, which is followed by a short $\alpha$-helix. These pack back onto Cdc42, contacting switches 1 and 2. A comparison of the three CRIB-Cdc42 structures shows that the effectors all contact the switch regions, $\alpha 1, \alpha 5$ and $\beta 2$ on Cdc42. The N-terminal regions of the CRIB effectors all interact with Cdc42 in a similar manner and indeed this is where they are most homologous. The C-termini of the effectors are not homologous and show some variation in their binding. PAK and WASP both adopt a $\beta$-hairpin followed by an $\alpha$-helix, however the way they interact with $\mathrm{Cdc} 42$ is quite different. The CRIB $\alpha$-helices both contact the helical portion of Cdc42 switch 2, but the Cdc42 helix is slightly shifted in the WASP complex such that it packs parallel to the WASP helix. In the PAK complex, the PAK helix packs at right angles to the Cdc42 helix making less extensive contacts. To compensate, the $\beta$-hairpin in in PAK makes more contacts with switch $2 \mathrm{Cdc} 42$ than does that of WASP (Figure 2.4). The ACK complex is significantly different to that of PAK and WASP. ACK forms a more regular $\beta$-sheet with $\mathrm{Cdc} 42$ and then proceeds to wrap around the body of Cdc42 making extensive contacts with switches 1 and 2 and forming an expansive binding interface.

Unlike ACK and WASP, which are specific for Cdc42 binding, PAK is able to bind to Rac1. Most of the regions that interact with the effectors are conserved between Cdc42 and Rac1. Leu174 Cdc42 is substituted by Arg174 in Rac1 and is one of the few contacts that would differ in the two complexes. In agreement with this, the L174A mutation reduces the affinity for ACK and WASP 30-fold but only affects PAK binding $~ 2.5 \mathrm{X}$ (Owen et al. 2000), suggesting that interactions with Cdc42 $\alpha 5$ are crucial to ACK and WASP and yet not so important for PAK binding. Two structures are available in the PDB describing Rac3 bound to the CRIB region of PAK1 and PAK4 (2QME and 2OV2). The Rac3-PAK1 structure shows some interesting differences with Cdc42-PAK1. The same intermolecular $\beta$-sheet is seen to form, however the flexibility of the PAK peptide after that becomes increasingly higher with the final 12 residues being invisible, so it is unclear whether PAK1 in 
complex with Rac3 forms the helix seen when it interacts with Cdc42. It seems likely that whatever structure the C-terminal region of the PAK1 GBD forms, it remains flexible while binding to Rac3.

The Rac3-PAK4 structure appears more similar to the Cdc42-PAK1 structure. PAK4 is seen to adopt a hairpin followed by a short helix, both of which pack back onto Rac3. The PAK1 GBD overlaps with a regulatory, autoinhibitory sequence (AID) in PAK1 (Zhao et al. 1998). Cdc42-Rac1 were known to activate the kinase activity of PAK1 and the mechanism underpinning this activation was elegantly demonstrated by (Lei et al. 2000) when they showed the sandwich formed by the PAK1 kinase domain, AID and GBD. Binding by Cdc42-Rac1 destabilizes contacts between the AID and the kinase domain, ultimately releasing the kinase domain, which is then phosphorylated and fully functional. The group II PAKs were not thought to be activated by binding of Rho-family small proteins and no AID had been identified in this subgroup (Jaffer \& Chernoff 2002). However new evidence and a re-evaluation of data indicates that PAK4 (and probably all group II PAKs) do have a fully functional AID (which is homologous to the group I AIDs) and are activated by Cdc42. The group II PAKs differ in being constitutively phosphorylated on their Aloop and as such are fully functional on $\mathrm{Cdc} 42$ binding. This is fully supported by the Rac3-PAK4 structure. A second group II structure is also available from the PDB (2ODB): Cdc42-PAK6 GBD. This shows the familiar architecture of the PAK1 GBD bound in the same manner to Cdc42, again supporting the idea that the group II PAKs are far more similar to the group Is in their activation by small $\mathrm{G}$ proteins than was initially thought.

\subsubsection{Cdc42-Par6}

Rho-family effector complexes have not been confined to canonical CRIB family effectors and the next structure to be solved involved an effector in possession of a 'semi-CRIB'. Par6, a protein involved in the regulation of cell polarity, has a partial CRIB motif that is insufficient alone to bind Cdc42. For small G protein binding, Par6 requires its adjacent PDZ domain (Ranganathan \& Ross 1997, Joberty et al. 2000). The crystal structure of Cdc42 in complex with the Par6 GBD (Garrard et al. 2003) shows that the semi-CRIB motif binds in an extended conformation and forms the 
anti-parallel $\beta$-sheet with $\beta 2$ of Cdc42 in a similar manner to the other CRIB effectors (Figure 2.5A). However the first $\beta$-strand of the Par6 PDZ simultaneously partners with the three-stranded $\beta$-sheet of the PDZ domain and the semi-CRIB, creating a 10 stranded $\beta$-sheet twisting its way through the middle of the complex. The orientation of the PDZ domain leaves it free to engage its ligand via $\beta \mathrm{B}$ and $\alpha \mathrm{B}$ on the opposite side of the complex to Cdc42. Unlike the ACK and WASP complexes, the extreme N-terminus of the Par6 GBD does not appear to be as important to complex formation and important contacts start in the CRIB motif. A key difference in this complex involves the lack of the two invariant histidine residues of the canonical CRIB in the semi-CRIB. These are seen to interact with Asp38 in all of the CRIB complexes, explaining why Asp38 mutations prevent CRIB effector binding to the Rho-family small $\mathrm{G}$ proteins. A proline replaces the first histidine in Par6, which is seen to have limited contacts to Tyr $40^{\text {Cdc } 42}$ but nothing to Asp38. The second histidine is then replaced with serine, the sidechain of which is orientated away from Asp38. These data explain why D38A Cdc42 retains the ability to bind Par6. The extended loop that then exists between the semi-CRIB and the PDZ restricts the contacts that Par6 can make with switches 1 and 2 on Cdc42 and as a result the complex buries only $\sim 1,100$ $\AA^{2}$ accessible surface area. Contacts are seen to Leu67 $7^{\mathrm{Cdc} 42}$ and Leu $70^{\mathrm{Cdc} 42}$ in switch 2 , in common with all other CRIB complexes. Overall, contacts are more polar than seen in the other CRIB complexes. Also, unlike the other CRIB family effectors, the CRIB motif in Par6 appears at least partially ordered in the free Par6 and may even form a structure quite close to that observed in the complex.

\subsubsection{Cdc42-IRSp53}

The most recent Cdc42-CRIB effector structure has proved to be the most divergent yet (Kast et al. 2014). IRSp53 has been proposed to be an effector protein for both Cdc42 and Rac1. It consists of an N-terminal BAR domain, a central region that contains a sequence with some homology to a CRIB motif that is closely followed by a proline-rich sequence and an SH3 domain towards its C-terminus. IRSp53 only maintains the first three invariant residues of the CRIB consensus sequence; it also has very low affinity for $\mathrm{Cdc} 42(5 \mu \mathrm{M})$ in contrast to the other consensus CRIB effectors, which have low $\mathrm{nM}$ affinities. The residues homologous to the CRIB (Val266-Pro270) make contacts that are seen in the other CRIB complex structures, 
interacting with residues in Cdc42 $\alpha 5$ e.g. Ile173 and Leu174. Concomitant with this, mutations S268A ${ }^{\mathrm{IRSp53}}$ and I267A $\mathrm{IRSp3}^{\mathrm{I}}$ abrogate binding to Cdc42. After Pro270 the homology at both the sequence level and the structural level diverges. The IRSp53 chain adopts an extended conformation that makes hydrophobic contacts with other regions of $\mathrm{Cdc} 42$, in fact crossing $\beta 2$ in a perpendicular manner rather than forming the classic intermolecular $\beta$-sheet (Figure 2.5B). Contacts with switches 1 and 2 are made by a short helix in IRSp53 (281-287) and the final 4 residues of the GBD. Mutation $\mathrm{F} 286 \mathrm{E}^{\mathrm{IRSp53}}$ is also sufficient to prevent binding to $\mathrm{Cdc} 42$.

Interestingly the IRSp53 GBD is thought to mediate an autoinhibitory interaction with the SH3 domain of IRSp53. The only consensus PxxP binding site in the GBD is 278PVPP-281. The structure shows that Pro278 and Pro281 are surface exposed and their mutation does not significantly affect $\mathrm{Cdc} 42$ binding. However data indicate that these mutations do interfere with the intramolecular SH3 interaction, suggesting that the IRSp53 GBD is capable of simultaneously binding both Cdc42 and an SH3 domain and therefore mediating both of the dual activation mechanisms of IRSp53. Despite the fact that IRSp53 has been shown to be a bone fide interactor for Rac (Miki et al. 2000), Kast et al. cannot identify an interaction with Rac1 and indeed the structure of Cdc42-IRSp53 shows binding regions on Cdc42 that would differ in Rac1 and possibly abrogate binding. It remains therefore to see whether IRSp53 can bind to Rac but via an alternative binding surface on the small $G$ protein.

\subsection{The Arf family}

The Arf family of small $\mathrm{G}$ proteins regulates vesicle formation and therefore intracellular trafficking. Although they do adopt the canonical Rossman fold of a G domain, they have been demonstrated to possess distinct properties compared to Ras. The Arf subfamily have an N-terminal extension over the canonical G domain that forms an $\alpha$-helix and are myristoylated at their $\mathrm{N}$-terminus, whereas Ras proteins are prenylated at the C-terminus. They also show differences between the GDP and GTP forms, with effectively four nucleotide sensitive regions, compared to two in Ras. The extra N-terminal helix shifts conformation in the GTP bound form and interacts with the membrane, where the myristoyl group also inserts. Regions analogous to switches 
1 and 2 change orientation as expected but Arf proteins also undergo an 'interswitch toggle'. This involves the central antiparallel $\beta$-sheet, which undergoes a significant large shift on GTP binding actually helping to displace the N-terminal helix. The second subfamily consists of the Arl proteins, which are not myristoylated but rather are acetylated at their $\mathrm{N}$-termini.

\subsubsection{Arl1-PDE $\delta$}

The structure of Arl2 complexed with PDE $\delta$ was the first effector complex to be solved for the Arf family (Hanzal-Bayer et al. 2002). PDE $\delta$ was discovered as the fourth subunit of cGMP phosphodiesterase in rod cells. Its role in the enzyme complex was unresolved at the time but it was known that it could extract the catalytic subunits from membranes. It was thought possible that PDE $\delta$ could act like the GDI proteins already known to exist for the Rho and Rab families of small $\mathrm{G}$ proteins. The structure of PDE $\delta$ was solved in complex with Arl2, so also showed for the first time the structure of activated Arl2. This was seen to have the canonical G domain fold and to possess the same extra $\mathrm{N}$-terminal helix observed in the Arf proteins (Figure 1.2). PDE $\delta$ bound to Arl2 in a GTP-specific manner and binding inhibited the release of nucleotide from the G-protein, thus strongly implicating PDE $\delta$ as an effector protein. PDE $\delta$ was seen to adopt an Ig-like fold with the overall $\beta$-sandwich fold identical to that already shown for RhoGDI. The interface between the two proteins comprised the all too familiar extended intermolecular $\beta$-sheet with $\beta 2 \operatorname{Arl} 2$ and $\beta 7$ PDE $\delta$ providing the contact edges (Figure 2.6A). This was also the first demonstration of the formation of a parallel intermolecular $\beta$-sheet being formed. The structure was also completely different to the Cdc42-RhoGDI (Hoffman et al. 2000) structure that was available, again implicating PDE $\delta$ as a genuine effector molecule for Ar12. Contacts on Arl2 included Asn37, Glu39, Leu48, Gly49, Phe50, Asn51, Ile52, Lys53, Thr54, Leu55, TRp65, Leu73, Tyr76, Asn79 and Tyr80; all residues lying in switch 1, switch 2 and the interswitch region. Hanzal-Bayer et al. went on to show that PDE $\delta$ actually also bound to Ras in a two-hybrid assay. Work has continued and it is now known that PDE $\delta$ does indeed bind to Ras proteins and is capable of extracting them from membranes and is involved in sustaining signalling from Ras proteins (Chandra et al. 2012). So PDE $\delta$ seems to have the properties of both an effector protein for Arf family proteins and a RasGDI. In fact Arl2 and Arl3 
act as GDI-like displacement factors for PDE $\delta$ by allosterically displacing its prenylated cargo (Ismail et al. 2011).

\subsubsection{UNC119 with Arl2 and Arl3}

Two further structures have emerged showing Arl2 and its close relative Arl3 in complex with UNC119. UNC119 is homologous to PDE $\delta$ and indeed adopts the same Ig-like fold. It is critical for targeting and trafficking myristoylated proteins to primary cilia and acts, like PDE $\delta$, as a solubilizing factor for lipidated cargo proteins. Interestingly Arl3 is capable of displacing cargo from UNC119 whereas Arl2 is not.

Similar to Arl2-PDES, UNC119 binds to Arl2 and Arl3 to form parallel intermolecular $\beta$-sheets (Figure 2.6B) and the contact edges involved $\beta 2$ of Arl2/3 and $\beta 7$ of UNC119. The interactions involve a mixture of polar and hydrophobic contacts. Residues on the small G proteins involved in contacts fall in switch 1, switch 2 and the interswitch region. The structure of Arl2-UNC119 is very similar to that of Arl2-

PDE $\delta$ : पढ口 $\square \square \square \square \square \square-$

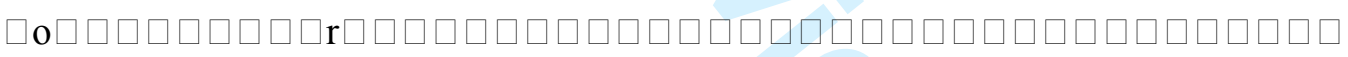

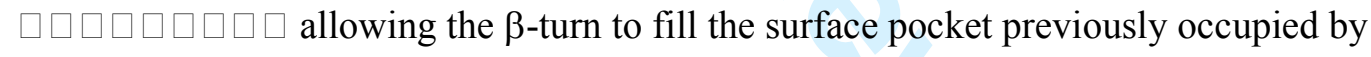
the N-terminal amphipathic helix. In the Arl3-UNC119 complex, the interswitch toggle is still seen, however the $\beta$-turn makes a distinctive move away from the hydrophobic surface pocket and towards UNC119. The Arl3 N-terminal helix therefore stays in its surface position. An examination of the hydrophobic cargo sequestration pocket of UNC119 reveals that it is in a more open conformation in the Arl3 complex, facilitating cargo release. The more conventional structure of the Arl2 interswitch in the Arl2-UNC119 complex does not promote opening of the farnesyl binding pocket and release of the cargo.

\subsubsection{Arf6-MKLP1}

The final effector complex mediated by an intermolecular $\beta$-sheet interaction for the Arf family proteins involves the outlying class III Arf protein, Arf6 and its effector MKLP1 (Makyio et al. 2012). The Arf binding domain of MKLP1 consisted of $5 \beta$ - 
strands, one short $\alpha$-helix and a long loop including a short $\beta$ A region (Figure 2.7). In the complex structure two MKLP1 GBDs come together to form a homodimer mediated in part by a small $\beta$-sheet formed between $\beta A 1-2$, this is combined with a more major interaction between the two $\beta 5$ strands to hold the MKLP1 dimer together. Two Arf6 molecules then bind the MLKP1 homodimer on either side. This structure is thought to be physiologically relevant. The four molecules together produce a superextended $\beta$-sheet of $22 \beta$-strands that runs through the whole complex. There were no known homologues for the MKLP1 GBD making this not only a novel small $\mathrm{G}$ protein binding module but also a unique protein fold. The Arf6 in the complex structure shows the classic Arf family-GTP bound conformation with an extended interswitch region and many of the Arf6 residues involved in the interaction with MKLP1 are similarly used to interact with another effector JIP4 (Section 3.5.3). The unique structure of the MKLP1 GBD allows it to make a series of hydrogen bonds with a highly conserved region of Arf6 known as the 'triad patch' (Phe47, Trp62 and Tyr77, for Ras numbering subtract 10), which is conserved within both the Arf and Rab-families. $\beta 2$ (switch 1) Arf and $\beta 5$ MKLP1 make the antiparallel $\beta$-sheet interaction held together by hydrogen bonds between Val45 $5^{\text {Arf }}$

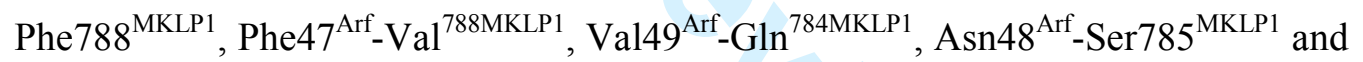
Glu50 ${ }^{\text {Arf }}$-Thr $779^{\text {MKLP1 }}$. A second set of interactions center around Arf6 switch 2. His $76^{\text {Arf }}$ makes a hydrogen bond with His $758^{\text {MKLP1 }}$ and a series of hydrophobic interactions also dominates the area mediated by Leu $73^{\text {Arf }}$, His $76^{\text {Arf }}$, Pro $720^{\text {MKLP1 }}$, Leu756MKLP1, His $758^{\text {MKLP1 }}$ and Ile $772^{\text {MKLP1 }}$. The final set of interactions at the triad patch involves Phe $47^{\text {Arf }}$, Tyr $77^{\text {Arf, }}$ Ala $743^{\text {MKLP1 }}$, Tyr754 ${ }^{\text {MKLP1 }}$ and Val786 ${ }^{\text {MKLP1 }}$ in a hydrogen bond network. The overall buried surface area of the complex is 2,241 $\AA$. In summary the complexes that Arf6 makes with its effector proteins are significantly different to one another so far and although the Arl proteins are known to make intermolecular $\beta$-sheet complexes with its effectors these are all parallel interactions while Arf6 forms an anti-parallel interactions.

\section{Effectors binding via a helical pair.}


The largest structural group of effectors utilizes a pair of $\alpha$-helices as their major interaction motif to interact with the small $\mathrm{G}$ proteins. Helical pair effectors are represented in 4 of the 5 families of small $G$ proteins, the exception being Ran. The plasticity of helical interactions when compared with the intermolecular $\beta$-sheet interactions already described means that all possible orientations of helical pairs are observed in these complexes (Figure 3.1). There are four classes of anti-parallel helical pair, based on the relative positions of the $\mathrm{N}$ - and $\mathrm{C}$-terminal helices and the loop that joins them. In addition, there are two classes of parallel helical pair. The 6 classes will be discussed separately and are named according to the name of their panel in Figure 3.1

\subsection{Type B}

This class of helical pair has only been observed in one effector complex so far. The RalA/B effector RLIP76 (or RalBP1) contains a coiled-coil that is sufficient for tight binding $\left(K_{\mathrm{d}} \sim 200 \mathrm{nM}\right)$ to the Ral proteins (Fenwick et al. 2010). This interaction is unique, in that it is the only known effector for a Ras family $G$ protein that binds using an anti-parallel coil-coiled. The RLIP76 coiled-coiled comprises two $\sim 25$ residue $\alpha$-helices, which interact with both switch regions of RalB (Figure 3.2). As the RLIP76 is a coiled-coil, the helices are wrapped around each other, so that although at the loop between the helices the $\mathrm{N}$-terminal $\alpha$-helix is above the $\mathrm{C}$ terminal helix in this orientation, at the opposite end their positions are reversed. This structure does, however, belong in the B class, since the majority of the interactions made with the switches involve RLIP76 residues closer to the inter-helix loop. The orientation of the helices with respect to the $\mathrm{G}$ domain is also different to most of the helical pairs (Figure 3.1), being tilted down on the right, rather than pointing upwards.

\subsubsection{RalB-RLIP76}

The RLIP76 Ral binding domain forms a coiled-coil in the absence of RalB and essentially has the same structure in the free and the bound forms. RalB, like most small G proteins, undergoes reorientation around the switch regions, which become fixed compared to the free RalB structure (Fenwick et al. 2009). The N-terminal portion of switch 1 does not make any contacts with RLIP76, but residues 48-52 (Ras equivalent 37-41), which are towards the C-terminus of switch 1 , interact with the 
RLIP76 coiled-coil. Ala48 $8^{\text {RalB }}$ packs against Leu409 $9^{\text {RLIP76 }}$ and His413 ${ }^{\text {RLIP76 }}$ in the Nterminal helix but the remainder of the switch 1 residues, at the start of the second $\beta$ strand, only contact the C-terminal RLIP76 helix. Asp49 ${ }^{\text {RalB }}$ forms a salt bridge with Lys $440^{\text {RLIP76 }}$, Tyr51 ${ }^{\text {RalB }}$ forms a hydrogen bond with Arg444 ${ }^{\text {RLIP76 }}$ and Arg52 ${ }^{\text {RalB }}$ contacts the backbone of Arg434 ${ }^{\mathrm{RLIP} 76}$. Switch 2 makes much more extensive contacts than switch1, interacting with both $\alpha$-helices of the RLIP76 coiled-coil. Asp $74^{\text {RalB }}$, Tyr75 $^{\text {RalB }}$, Ala76 ${ }^{\text {RalB }}$ Ala-77 ${ }^{\text {RalB }}$ and Ile $78^{\text {RalB }}$ (equivalent to Ras 63-67) make multiple contacts with His $413^{\text {RLIP76 }}$, Leu $416^{\text {RLIP76 }}$ and Gln-417 $7^{\text {RLIP76 }}$, which are all in the first $\alpha$-helix of the coiled-coil. Asn $81^{\text {RalB }}$ and Tyr $82^{\text {RalB }}$ at the C-terminus of switch 2 contact residues in both RLIP76 $\alpha$-helices, while residues just after switch 2 (Arg-64 ${ }^{\text {RalB }}$ and Ser85 ${ }^{\text {RalB }}$ ) exclusively contact the C-terminal helix of RLIP76. In addition to the contacts with the switch regions, two residues between the switches, Asp65 and Leu67 (Ras 54 and 56) are important for the interaction with RLIP76. Asp $65^{\text {RalB }}$ forms a salt bridge with R434 ${ }^{\text {RLIP76}}$, while Leu67 ${ }^{\text {RalB }}$ makes extensive hydrophobic contacts with Trp430 ${ }^{\mathrm{RLIP} 76}$. All of the residues in RalB that contact RLIP76 are conserved in its sister protein RalA and it is thus of no surprise that RalA and RalB bind to RLIP76 with similar affinities in vitro. Ras itself does not bind to RLIP76, even though most of the switch regions and residues that contact RLIP76 are conserved between H-Ras and RalB. The discriminatory residues have been pinpointed as Lys47 and Ala48 in RalB, whose H-Ras equivalents are Ile37 and Glu37. The H-Ras double mutant I36K/E37A is able to bind to RLIP76, while the RalB K47I/A48E mutant has lost RLIP76 binding (Bauer et al. 1999). The RalB K74A mutation had little effect on binding, while the A48G mutant affinity was reduced 5-fold (Fenwick et al. 2010). Other RalB mutations in the interface that reduce binding of RLIP76 include Y82A (>5-fold), L67A (>5-fold) and I76A (4fold).

\subsection{Type C}

This type of $G$ protein-helix interaction has been found in both Rab and Arf family effectors and is exemplified by two complexes: that formed between Arf1 and the GAT domain of GGA1 and that of Rab11 with the Myo5B globular tail domain (GTD) (Figure 3.3). Although these two complexes are in the same structural class, their helices are in different orientations with respect to the $G$ protein and to each 
other. In the Arf1-GGA1 structure, the GGA1 N-terminal helix is almost horizontal in this orientation, while the $\mathrm{C}$-terminal helix slopes upwards. This leads to a $\sim 45^{\circ}$ angle between the two helices of the effector. In the Rab11-Myo5B structure, the two helices are sloping downwards in this orientation and are almost parallel. The Cterminal helix is slightly tilted however, so that its C-terminal end is going back into the page from this viewpoint. We have also included the structure of Rab8 with the 'super-effector' LidA, from Legionella pneumophila. This effector has high affinity for both GDP- and GTP-bound forms of the Rab proteins but is thought to bind to the active, GTP-bound form more tightly. The LidA protein includes two $\alpha$-helices that are in the Type $\mathrm{C}$ class of helical pair effectors. These helices form a coiled-coil and are oriented so that they are approximately parallel to each other and in a similar orientation to the scheme in Figure 3.1 i.e. they are tilted upwards on the right hand side in this orientation.

\subsubsection{Arf1-GGA1}

GGA1 is an adaptor protein involved in vesicle transport between membrane-bound organelles. There are several structures of the free GGA1 GAT domain (Suer et al. 2003; Collins et al. 2003; Zhu et al. 2003; Shiba et al. 2003), showing that it contains $4 \alpha$-helices arranged into two small subdomains, one comprising helices 1 and 2 and the other a triple helical bundle containing helices $2-4$. The two subdomains are connected by helix 2. The short helix 1 and the N-terminus of helix 2 have high temperature factors and vary between the different structures, suggesting that they are flexible in solution. The structure of the GGA1 in complex with Arf1 could only be achieved by co-crystallizing a fragment of the GAT domain, containing helix 1 and the N-terminus of helix 2 (Shiba et al. 2003). This fragment appears to become more rigid in the complex structure but presumably there is some flexibility in its connection with the rest of the domain that prevented crystal formation of the full GAT domain in complex. The truncated GGA protein and the full GAT domain have similar affinities for Arfl (1.1 and $1.4 \mu \mathrm{M}$ respectively).

The GGA1 GAT helices interact with both switch regions of Arl1 and with the interswitch $\beta$-sheet (strands $\beta 2$ and $\beta 3$, see Figures 1.1 and 1.2) and it was shown that the Arf1 structure does not change on complex formation. The interface is mainly 
hydrophobic but also contains three hydrogen bonds. In general, the N-terminal helix in the GG1 helical pair interacts with switch 2, while the C-terminal helix interacts with switch 2. Ile49, Gly50 and Phe51 in switch 1 (Ras equivalent 36-38) are the switch 1 residues involved in contacts. Ile $49^{\text {Arfl }}$ mainchain forms a hydrogen bond with Lys $198^{\mathrm{GGA} 1}$ and forms hydrophobic contacts with Val201 ${ }^{\mathrm{GGA} 1}$, Gly50 ${ }^{\mathrm{Arfl}}$ packs against Ile $197^{\mathrm{GGA} 1}$ and Phe $51^{\mathrm{ARF} 1}$ mainchain forms a hydrogen bond with Asn $194^{\mathrm{GGA} 1}$ and also contacts Leu182 ${ }^{\mathrm{GGA} 1}$. In the interswitch region, W66 ${ }^{\mathrm{ARF} 1}$ (Ras equivalent 56) contacts $\mathrm{L} 190^{\mathrm{GGA} 1}$ in the C-terminal GGA1 helix. The switch 2 contacts involve Lys $73^{\mathrm{Arf1}}$ and Ile $74^{\mathrm{Arf} 1}$, which contact Val201/Gln205 ${ }^{\mathrm{GGA} 1}$ and Val201 ${ }^{\mathrm{GGA} 1}$ respectively; Leu $77^{\mathrm{Arfl}}$ which forms hydrophobic interactions with Phe169/Leu178/Ile197 ${ }^{\mathrm{GGA} 1}$; His80 ${ }^{\mathrm{Arf1}}$ contacts Ala179/Leu182 ${ }^{\mathrm{GGA} 1}$ and Tyr82 ${ }^{\mathrm{Arf} 1}$ interacts with Leu182 ${ }^{\mathrm{GGA} 1}$. The equivalent residue numbers in Ras would be 63, 64, 67,70 and 72 .

\subsubsection{Rab11-Myo5B}

The Myo5 motor proteins are involved in transport of a number of cargoes and are linked to recycling membrane compartments via interactions between their globular tail domains and Rab proteins. The globular tail domain structure has been solved and is made up of $12 \alpha$-helices (H1-H12), which form two subdomains connected by the long H5 helix (Pylypenko et al. 2013). Rab11 binds directly to Myo5B via one of these subdomains, primarily contacting via helices $\mathrm{H} 8$ and $\mathrm{H} 9$ of the Myo5B protein, although one residue in $\mathrm{H} 10$ and several in the H5-H6 interhelical loop also make contacts (Figure 3.4A). The binding interface is made up of two hydrophobic patches surrounded by polar residues. The polar residues include Lys $13^{\text {Rab11 }}$, which forms a hydrogen bond with Gln1628 ${ }^{\mathrm{Myo5B}}$ and Arg33 ${ }^{\text {Rab11 }}$ whose hydrocarbon portion packs against that of $\operatorname{Arg} 1724^{\mathrm{Myo5B}}$. The switch 1 interactions are hydrophobic and involve packing between Ile44 ${ }^{\text {Rab11 }}$ and Leu1763/Leu1749/Gln1745 ${ }^{\text {Myo5B }}$; Val46 ${ }^{\text {Rab11 }}$ whose backbone hydrogen bonds to $\operatorname{Gln} 1748^{\mathrm{Myo5B}}$ and whose sidechain packs against Met1710 ${ }^{\mathrm{My} 5 \mathrm{~B}}$ and Phe48 ${ }^{\mathrm{Rab} 11}$, which contacts Ile1627/Tyr1714 ${ }^{\mathrm{Myo5B}}$. These three switch 1 residues are equivalent to $\operatorname{Ras} 36,38$ and 40 . The interswitch region also makes interactions: Thr50 Rab11 hydrogen bonds to Glu1721 ${ }^{\mathrm{My} 05 \mathrm{~B}}$, Gln63 ${ }^{\text {Rab11 }}$ contacts Ile1627/Met1710 ${ }^{\text {Myo5B }}$ and Trp65 ${ }^{\text {Rab11 }}$ interacts with Ile1627/Leu1630/Met1710 ${ }^{\text {Myo5B }}$. The interactions with switch 2 are extensive: Glu71 ${ }^{\text {Rab1 }}$ (equivalent to Ras residue 62) 
forms a salt bridge with Lys $1750^{\mathrm{Myo5B}}$ and Tyr $73^{\text {Rab11 }}$ packs against Leu1763 ${ }^{\mathrm{My} 05 \mathrm{~B}}$ : these Myo5B residues are both in the loop between H9 and H10. The majority of the switch 2 hydrophobic interactions involve Trp1706 and Met1710 in Myo5B helix H8 (the N-terminal helix in the helical pair) and Ala75, Ile76, Thr77 and Tyr80 from Rab11.

The interactions between Myo5B and Rab11 involve the hydrophobic triad patch, Phe48, Trp65 and Tyr80, which is conserved in all Rab proteins and is thought to be important for Rab effector specificity (reviewed in (Khan \& Ménétrey 2013)). Interestingly, the structure of Rab11 in the Myo5B complex shows that the switches are in a dramatically different conformation than in several Rab11 structures previously determined. Switch 1 shifts by $1.8 \AA$ and the switch 2 helix forms only a single turn of $3_{10}$ helix, the remainder being in an extended conformation. The reorientation of Rab11 to bind Myo5B is indicative of an induced fit mechanism of binding between these two proteins and may thus help to explain the selectivity of Myo5B binding. The amino acids involved in direct contacts with Myo5B are conserved between the Rabs but Myo5D does not bind to all Rab proteins. GTPbound Rab11 appears to have an unusual conformation around the switches and the hydrophobic triad (Pylypenko et al. 2013), which presumably plays a role in the specificity. Nevertheless, the conservation of the interacting residues even in noninteracting Rab proteins illustrates how difficult it is to predict binding based on sequence alone.

\subsubsection{Rab8-LidA}

LidA is injected into the host cell cytoplasm at the beginning of Legionella pneumophila infection and is important for interfering with the host vesicle trafficking system by binding to Rab proteins. The central domain of LidA binds to Rab proteins and is made up of $7 \alpha$-helices and 5 anti-parallel $\beta$-sheets (Schoebel et al. 2011). Two anti-parallel coiled-coils form a helical platform at the base of the protein and two parallel pillars extend more or less perpendicularly from the platform. The regions of LidA that interact with the Rab8 switch regions include a helical pair within the platform, comprising helices H4 and H5 (residues 368-449) and it is these helices that contact the Rab8 hydrophobic triad (Phe45, Trp62 and Tyr77) as well as making 
several contacts with the switch regions. The LidA and Rab8 interface includes two central hydrophobic patches, the first of which is mostly made up from LidA helices H4 and H5, surrounded by more polar contacts with the LidA pillars. Switch 1 contacts helix $\mathrm{H} 5$ and helix H7: residues Phe $37^{\text {Rab8 }}$ and Ile $38^{\text {Rab8 }}$ contact Leu436/Tyr243 ${ }^{\text {LidA }}$ and Leu428 $8^{\text {LidA }}$ respectively, while Ile41 ${ }^{\text {Rab8 }}$ and Ile $43^{\text {Rab8 }}$ interact with Tyr532 $2^{\text {LidA }}$ and Leu548/Ile552 ${ }^{\text {LidA }}$. These Rab8 residues are equivalent to residues 32, 33, 36 and 38 in Ras. The hydrophobic triad contacts are: Phe45/Trp62 $2^{\text {Rab8 }}$ to Ile413/Leu428 $8^{\text {LidA }}$ and Tyr77 $7^{\text {Rab8 }}$ to Ile406/His431 ${ }^{\text {LidA }}$. Switch 2 residues of Rab8 make extensive contacts with helices 4 and 5 of LidA as well as helix 6 in one of the pillar structures.

In total, LidA utilizes 4 helices to bind to Rab8 and buries an extensive interface in the contact site (Figure 3.4B). Outside the switch regions, Rab8 residues Thr91, Asn92, Arg104 and Gln130 also make contacts with LidA. This leads to its having a remarkably high affinity for Rab8, in the $\mathrm{pM} K_{\mathrm{d}}$ range, in fact too high for accurate measurement. As its interaction involves the switch regions, it is expected that LidA is selective for the GTP-bound, active form, of Rabs and this seems to be the case for Rab1, whose LidA complex structure is very similar (Cheng et al. 2012), albeit with a lower affinity $(7.5 \mathrm{nM})$. Surprisingly, Rab1 - GDP binds to LidA with a similar affinity and the structure shows that in this complex the Rab switches adopt a GTP-like conformation. LidA appears to interact with several other Rab proteins but with lower affinities $(250 \mathrm{nM}$ to $7 \mu \mathrm{M})$. A mutagenesis approach was attempted to pinpoint the residues that determine the specificity of the interaction but was not successful (Cheng et al. 2012).

\subsection{Type D}

This anti-parallel arrangement is the most common of the coiled-coil interactions that has been observed and includes examples from the Rab, Arf and Rho families (Figure 3.5). The Rab effectors Exophilin1 (or Rabphilin3A), Exophilin4 (Slp2-a) and Melanophilin (Slac2-a) bind to Rab proteins using a long N-terminal helix and a shorter C-terminal helix. The helices all slope upwards from left to right in this orientation. The other Rab effectors in this class include Rab-7-RILP, which forms a $2: 2$ tetramer, so that the two helices in the helical pair come from two different RILP 
molecules. Nevertheless, they are anti-parallel and fit into this structural class. Rab4 and Rab22 bound to their interacting regions from Rabenosyn-5 are also within this structural class. The helices are oriented so that they are almost horizontal in this orientation and even though they come from different regions from the Rabenosyn-5 molecule their structures are remarkably similar. The GRIP domain from golgin-245 binds to Arl1 using two short $\alpha$-helices, which interact closely with the switch regions, so that their position is directly in front of the Arl1 molecule in this orientation, rather than interacting with one side of the $\mathrm{G}$ domain. The helices are also oriented so that they are pointing upwards from left to right, similar to that in the schematic (Figure 3.1D). The examples from the Rho family that fall into this structural class include the HR1 domains from PRK1, whose structures have been solved bound to RhoA (HR1a) and Rac1 (HR1b). The HR1 domain forms two long $\alpha$-helices in a coiled-coil, which interact similarly with the Rho proteins and are also oriented to point upwards towards switch 2 . The interaction between the diaphanousrelated formins and the Rho proteins also include a helical pair that loosely belongs in this class of interaction. In this case the N-terminal helix lies across the G protein in a similar orientation to that in Figure1D but the C-terminal helix points downwards from left to right, so that the angle between the helices is almost $90^{\circ}$.

\subsubsection{Rab3A-Exophilin1, Rab27A-Exophilin4 and Rab27B-Melanophilin}

The Rab3 and Rab27 proteins are involved in exocytosis and their effector proteins generally contain a conserved Rab27-binding domain (RBD27). The RBD27 from Melanophilin and Exophilin4 exclusively recognize Rab27, whereas Exophilin1 also binds to Rab3. Despite their sequence homology, the RBD27 domains from these three proteins have structural differences (Figure 3.6). The two helices of Exophilin4 are connected by a simple short loop, whereas the connection between the helices of Exophilin1 and Melanophilin contains a domain that binds to two $\mathrm{Zn}$ ions. The Nterminal helix of these three effector domains adopts a similar position in the three structures and in contrast to Rab11 described above (3.2.2), the Rab3A and Rab27 proteins contain a well-ordered helix in switch 2, packed against the $\mathrm{N}$-terminal helix of the effector. In contrast, the C-terminal helix of these RBD27 domains exhibits more variation (Figure 3.6). In Exophilin1, the C-terminal helix is short and then unwinds into an extended region that interacts with the Rab protein (Ostermeier \& 
Brunger 1999). In Melanophilin there are two C-terminal helices, oriented so that they form a single $\alpha$-helix interrupted by a short, five residue break that allows the $\mathrm{C}$ terminal portion to shift closer to the G domain (Kukimoto-Niino et al. 2008). In Exophilin4, the C-terminal helix is shorter and does not interact with either of the switch regions of Rab27A. Extra contacts with switch 2 are made with the long Nterminal helix from another Exophilin4 molecule in the asymmetric unit, although this interaction is thought to be non-specific and arises from crystal packing (Chavas et al. 2008).

In the Rab3A-Exophilin1 complex, the long N-terminal helix of the effector makes the majority of the contacts with the switch regions and the hydrophobic triad residues (Ostermeier \& Brunger 1999). Within switch 1, Ile57 ${ }^{\text {Rab3A }}$ and Phe59 $9^{\text {Rab3A }}$ make hydrophobic interactions with Val57 ${ }^{\text {Exo1 }}$ and Val57/Ala61 ${ }^{\text {Exo1 }}$, while Asp58 Rab3A forms a salt bridge with Arg60 ${ }^{\text {Exol }}$. In the interswitch region the hydrophobic triad residue $\operatorname{Trp} 76^{\mathrm{Rab3A}}$ contacts Ala61 ${ }^{\text {Exol }}$. In switch 2 there are hydrogen bond/salt bridges between Arg83 ${ }^{\text {Rab3A }}$ and Glu50 $0^{\text {Exo1 }}$ and between Tyr84 ${ }^{\text {Rab3A }}$ and Glu50 ${ }^{\text {Exo1 }}$ and hydrophobic contacts between Ile $87^{\text {Rab3A }}-$ Ile $54^{\text {Exo1 }}$ and Ala90 ${ }^{\text {Rab3A }}$ and Leu163 ${ }^{\text {Exo1 }}$, which is in the $\mathrm{C}$-terminal extended region of the effector. The final residues of the hydrophobic triad, Tyr91 ${ }^{\text {Rab3A }}$ interacts with Ile56 ${ }^{\text {Exo1 }}$. Similarly, in Rab27AExophilin4, the switch residues, Asp45 and Phe46 in switch 1, the interswitch/hydrophobic triad Trp73 and switch 2 residues, Arg80, Phe81, Leu84 and Phe88 (hydrophobic triad) also contact exclusively the N-terminal helix of the effector (Chavas et al. 2008). In the Rab27B-melanophilin complex, because the effector C-terminal helix is extended into a longer interrupted helix, switch 2 interactions are also made by the C-terminal regions (Kukimoto-Niino et al. 2008). Hence, in switch 1, Ile44 ${ }^{\text {Rab27B }}$, Asp45 Rab27B and Phe46 ${ }^{\text {Rab27B }}$ contact the N-terminal helix of melanophilin, while within switch 2 , Arg-80/Phe- $81^{\text {Rab27B }}$ also contact the Nterminus of melanophilin, while Leu- 84 and the hydrophobic triad residue Phe- 88 contact residues in the $\mathrm{C}$-terminal helix.

Outside the switch regions, the Rab27 proteins make several similar interactions: Tyr6/Tyr8, Met93, Tyr122 and Met-85 all make extensive contacts with the C- 
terminal helix of the effector protein. In Rab3A the equivalent residues, Phe19, Tyr21, Met96, Trp125, Met187 all contact the short C-terminal helix.

Exophilin1 binds to Rab3A and Rab8, as well as to Rab27A, while Exophilin4 and Melanophilin exclusively recognize the Rab27 isoforms. How is this specificity achieved? As is generally the case, the switch regions are relatively conserved in Rab3A and the Rab27 proteins, especially the residues that bind to the long Nterminal helix of these effectors. Two residues in Rab27A were selected: Tyr6 ${ }^{\text {Rab27A }}$ forms a hydrogen bond with Glu32 of Melanophilin and Asp91 ${ }^{\text {Rab27A }}$ forms a salt bridge with $\operatorname{Arg} 29^{\mathrm{Mel}}$ and a hydrogen bond with the Gly-133 ${ }^{\mathrm{Mel}}$ mainchain. When these residues were changed to their Rab3A counterparts (Phe19 and Gly94 respectively, the binding of Rab27A to Melanophilin was significantly reduced (Kukimoto-Niino et al. 2008). Two further mutations were also made in residues involved in hydrophobic interactions, Leu84 and Phe88 from switch 2, which are Ile87 and Tyr91 in Rab3A, the interaction between Rab27A and Melanophilin was abolished. The Rab3A residues were also mutated to their Rab27A counterparts: when the mutations were made singly, there was little effect on Rab3A's ability to bind to Melanophilin, but when the four residues were mutated together (i.e. Rab3A F19Y, I87L, Y91F and G94D), binding to Melanophilin was detectable, although it was weaker than the Rab27A binding.

\subsubsection{Rab7-RILP}

Rab7 is involved in endocytosis and regulates traffic between endosomes and lysosomes. RILP (Rab7-interacting lysosomal protein) contains a Rab7-binding region that is composed of just $2 \alpha$-helices, which form a homodimer in the crystal that interacts with $2 \mathrm{Rab} 7$ molecules (Wu et al. 2005). The presence of this heterotetramer in solution was verified by gel filtration chromatography. The Rab7 protein in the complex structure, like Rab11, has a switch 2 region that forms an extended loop rather than a regular $\alpha$-helix. The $\mathrm{N}$-terminal helix and the $\mathrm{C}$-terminal helix that make up the helical pair originate from the two different RILP molecules in the dimer. They are, however, anti-parallel and make similar interactions to the other helical pairs described. It is clear from Figure 3.5B that only one end (the $\mathrm{N}$-terminus) of the C-terminal helix of the RILP is close to the Rab7 protein. 
Switch 1 makes a few contacts with the N-terminal RILP helix: Asp $44^{\text {Rab7 }}$ forms a salt bridge with Lys $259^{\mathrm{RILP}}$ and the hydrophobic triad residue Phe $45^{\mathrm{Rab} 7}$ contacts the methylene groups of the same RILP Lys. Within the interswitch region, Gln60 $60^{\text {Rab7 }}$ contacts the C-terminal helix via Met305 ${ }^{\mathrm{RILP}}$, making a hydrogen bond with the mainchain and the hydrophobic triad residue Trp62 $2^{\text {Rab7 }}$ interacts with Asn256 ${ }^{\text {RILP }}$. In switch 2, Ser72 ${ }^{\text {Rab7 }}$ forms a hydrogen bond with Glu249 ${ }^{\text {RILP }}$ and Leu $73^{\text {Rab7 }}$ contacts Glu249 ${ }^{\text {RILP }}$ and Leu252 $2^{\text {RILP }}$. More interactions are made in the loop C-terminal to switch 2 that includes the third residue of the hydrophobic triad, Phe77, which packs behind switch 1. Arg79 ${ }^{\mathrm{Rab} 7}$ contacts the mainchain of the C-terminal helix, around Gly $307^{\text {RILP }}$ and Asp $82^{\text {Rab7 }}$ forms a salt bridge with Lys $304^{\text {RILP }}$.

Outside the switch regions, residues in the $\mathrm{N}$ - and $\mathrm{C}$-terminus of Rab7 contact RILP. Ser3, Leu8 and Lys10 in Rab7 contact residues in both the N-and C-terminal RILP helices. At the C-terminus of Rab7, Val180 and Leu182 contact the C-terminal RILP helix, while Tyr183 and Glu185 contact the N-terminal RILP helix.

Yeast two hybrid experiments were used to show that the RILP protein dimerizes in this system as well as in vitro and mutation of residues in the RILP:RILP interface prevented the dimerization (Wu et al. 2005). The importance of the RILP dimer for Rab7 interactions was also demonstrated, since the monomeric mutants were also unable to bind to Rab7. Rab7 mutants L8A, K10A, V180A also prevented the Rab7 RILP interaction, emphasizing the role of these regions outside the switches in the binding affinity. Other mutations that abrogated binding were D44A, F45A and D82 within the switches.

\subsubsection{Rabenosyn-5-Rab4 and Rabenosyn-5-Rab22}

Rabenosyn-5 (Rbsn) is an effector that contains two distinct Rab-binding regions, which permit the co-localization of two Rab proteins within the endosomal system. A panel of Rab proteins were tested for binding to the individual Rab-binding regions, which have similar structures (Eathiraj et al. 2005) and it was found that despite their homology the two coiled-coils in Rabenosyn-5 recognize distinct Rab subsets. The first Rab-binding region, residues 440-503, binds tightly to Rab4 and Rab14 (around $10 \mu \mathrm{M} K_{\mathrm{d}}$ ), while Rbsn residues 728-784 binds tightly to Rab5, Rab22 and Rab24 (2- 
$\left.10 \mu \mathrm{M} K_{\mathrm{d}}\right)$ and more weakly to Rab14 $(\sim 50 \mu \mathrm{M})$. The basis for the specificity of the two Rab-binding regions in Rbsn was established from the structures of Rbsn (440503) with Rab4 and Rbsn (728-784) with Rab22 and the differences between these homologous structures offer interesting insights. In Rab4, residues in helix $\alpha 1$, Leu25, His26 and Ile29 make hydrophobic contacts with Rbsn, which are not conserved in Rab22-Rbsn. The interactions in the switch regions also involve the less conserved residues. In Rab4, Ile41, Val43, Glu44 and Phe45 contact Rbsn, while in Rab22 Ile38 (equivalent to Rab4 Ile41) does not interact with the effector, although Ala40 and Ser41 contact, as does the conserved Phe42. The interswitch region contacts are partly conserved in the Rabs: in Rab4 Ser47, Lys58 and Gln60 make polar contacts with Rbsn, and in Rab22, Thr44 and Lys55 also contact polar residues, although Leu57 (equivalent to $\mathrm{Gln} 60^{\mathrm{Rab} 4}$ ) forms a hydrophobic interaction. The conserved Trp62 $2^{\text {Rab4 }} / \operatorname{Trp} 59^{\text {Rab22 }}$ contacts a conserved Asn in the Rbsn helices. The contacts in switch 2 are also only partly conserved: Arg6 $69^{\text {Rab4 }}$ forms a salt bridge, Phe $70^{\text {Rab4 }}$ and Val73 ${ }^{\text {Rab4 }}$ form hydrophobic contacts and Ser $76^{\text {Rab4 }}$ forms a hydrogen bond. In contrast, Arg66 ${ }^{\text {Rab22 }}$ packs against hydrophobic residues, Phe $67^{\text {Rab22 }}$ does not form close contacts, Leu70 ${ }^{\mathrm{Rab} 22}$ forms hydrophobic contacts and Met73 ${ }^{\mathrm{Rab} 22}$ contacts polar residues in Rbsn. The third of the hydrophobic triad, Tyr $77^{\text {Rab4 }} /$ Tyr $74^{\text {Rab22 }}$ hydrogen bonds to Asn/Gln residues in the Rbsn. In summary, the hydrophobic triad interactions are well conserved but the details of the interactions with the switch regions show several differences. There are also very few interactions outside the switches. Mutational analysis of the Rab5-Rbsn (728-784) interface showed that changing residues equivalent to Ser $41^{\text {Rab22 }}$ or Met $73^{\text {Rab22 }}$ reduced the binding to Rbsn 728-784.

\subsubsection{Arl1-GRIP}

Arl1 is a Golgi-associated Arf-like protein, which associates with two effectors on the Golgi apparatus known as golgins. The golgins are large, coiled-coil proteins involved in Golgi maintenance and trafficking that interact with Arl1 via a conserved GRIP domain. The GRIP domain includes three $\alpha$-helices but only two of them are involved in interactions with Arll (Panic et al. 2003; Wu et al. 2004). The GRIP domain forms a homodimer in the Arl1-GRIP structure, but all interactions with the G protein are mediated by one monomer: the dimer interface involves all three helices, which 
interact via an extensive hydrophobic interface that is distinct from the Arl-interacting surface. The dimer persists in solution, as shown by gel filtration and analytical ultracentrifugation.

The GRIP domain N-terminal helix makes most of the contacts with switch 1, while the C-terminal helix in the helical pair makes switch 2 contacts. Only a single residue in the GRIP domain, Tyr2177, contacts both switches and mutation of this residue to Ala prevents Golgi targeting, which is one of the consequences of the Arl1-GRIP interaction. Tyr2177 extends into a pocket on the Arl1 protein and is surrounded by hydrophobic residues from switch 1 (Phe51), the interswitch region (Leu66) and switch 2 (Ile74, Tyr77 and Ty-81). Other switch 1 residues involved in the interaction with Tyr2177 are Ile $49^{\text {Arl1 }}$ and Gly50 ${ }^{\text {Arl1 }}$, equivalent to 36 and 37 in Ras. In the interswitch region, Gln $64^{\text {Arl } 11}$ forms a hydrogen bond with Glu2190 GRIP and Trp66 ${ }^{\text {Arl1 }}$ contacts Glu2190/Met2194 ${ }^{\text {GRIP }}$, while in switch 2 Cys80 $0^{\text {Arl1 }}$ is packed against Val2197/Lys2196/Thr2200 GRIP .

The pocket that accommodates Tyr $2177^{\text {GRIP }}$ may be the key to Arll selectivity, since a pocket that is wide enough for the Tyr seems to be unique to Arl1. Most of the Arf family proteins contain a Leu at position 77, whereas Arl1-3 have a Tyr. In Arl2, however, Ile74 is replaced by a Leu, which may alter the geometry of the pocket and prevent GRIP binding. Cys80 is also not conserved in other Arl proteins and its mutation to His (the residue found in this position in Arfs) prevented binding to GRIP domains (Lu \& Hong 2003). A mutagenesis study has also shown that the GRIP domain dimerization is essential for interaction with Arl1, as well as for its localization to the Golgi ( $\mathrm{Lu}$ et al. 2006). This is surprising, since only one of the GRIP monomers is involved in contacting each Arl1 protein. Presumably the dimerization of the GRIP domain is necessary to form a platform that presents the helical pair in an orientation that is competent for binding to Arl1.

\subsubsection{PRK1 HR1a-RhoA and HR1b-Rac1}

The PRK1 serine/threonine kinase is involved in cytoskeletal organization, linking insulin receptor signaling with actin rearrangements, as well as playing a role in cell division. It contains three HR1 domains at the N-terminus and of these, the first, HR1a, binds to RhoA, RhoB, RhoC and Rac1 with affinities ranging from 30-200 
nM(Owen et al. 2003; Hutchinson et al. 2013). The second HR1 domain, HR1b, binds tightly to Rac1 $\left(K_{\mathrm{d}} 70 \mathrm{nM}\right)$ but not to RhoA (Owen et al. 2003). The structures of HR1a-RhoA and HR1b-Rac1 show that the HR1 domains contact the switch regions utilizing the conserved residues in the coiled-coil to contact the switches (Maesaki et al. 1999; Modha et al. 2008). The majority of the interactions are made with the Nterminal helix of the HR1 coiled-coil, which contacts both switches, while the Cterminal helix makes contacts in the centre of the interface with switch 2 only. Switch 1 residues Val36 ${ }^{\mathrm{Rac} 1} / \mathrm{Val} 38^{\mathrm{RhoA}}$ and Phe $37^{\mathrm{Rac} 1} / \mathrm{Phe} 39^{\mathrm{RhoA}}$ are involved in hydrophobic interactions, while Asp38 $8^{\mathrm{Rac} 1} / \mathrm{Asp} 40^{\mathrm{RhoA}}$ and Asn39 $9^{\mathrm{Rac} 1} / \mathrm{Asn} 41^{\mathrm{RhoA}}$ form polar contacts with the exposed face of the N-terminal helix. Within switch $2, A s p 63^{\text {Racl }}$ forms a hydrogen bond, while Asp $65^{\text {RhoA }}$ forms a salt bridge and Tyr64 ${ }^{\text {Rac1 }} /{\text { Tyr } 66^{\text {RhoA }}}_{\text {form }}$ mixed hydrophobic and polar interactions. Arg66 ${ }^{\text {Racl }}$ forms a salt bridge with Asp $172^{\mathrm{HR} 1 \mathrm{~b}}$ in the C-terminal helix, whereas Arg68 ${ }^{\mathrm{RhoA}}$ forms a salt bridge with Glu $49^{\mathrm{HR} 1 \mathrm{a}}$ in the N-terminal helix. This rearrangement occurs due to the nonconservation of the acidic residues in the HR1 domains. Leu67/Leu69 and Leu70/Leu72 are in the center of the interface and both make a number of hydrophobic contacts with both HR1 domain helices. These are not all conserved but the plasticity of these hydrophobic interactions allows the subtle reorganization of the sidechains involved.

The importance of these contacts for the binding of the HR1 domains has been analysed by Ala-scanning mutagenesis. In the RhoA-HR1a interface, F39A, D65A, R68A and L69A mutations all reduced or abrogated binding (Hutchinson et al. 2011). In the Rac1-HR1b interface, only D63A and L67A showed inhibited interaction (Owen et al. 2003), suggesting that the energetic contributions of the Phe37 and Arg66 (Rac numbering) interactions are much less. Intriguingly, the Rac1-HR1b interaction has also been shown to be dependent on the C-terminal tail of the Rac1 protein, which is unusual in that it contains an uninterrupted stretch of six basic amino acids (Owen et al. 2003; Modha et al. 2008). Mutation of any of these basic residues reduced the affinity between Rac1 and HR1b (Owen et al. 2003) and the structure showed that the $\mathrm{C}$-terminus of Rac1 loops back, contacting the $\mathrm{G}$ domain at the $\mathrm{C}$ terminus of switch 2 and then making contacts with the residues in the HR1b Cterminal helix (Modha et al. 2008). 


\subsubsection{RhoC-mDia1, Cdc42-mDia1 and EhRho1-Ehformin1}

The diaphanous-related formins interact with the Rho family proteins via a GTPase binding domain at their $\mathrm{N}$-terminus, leading to remodeling of the actin cytoskeleton. This domain contains three $\alpha$-helices but the majority of interactions with the small $\mathrm{G}$ protein switches are made by just two of them, suggesting that they should be included within this structural class, although they are outliers. Structures have been solved of RhoC in complex with the N-terminus of Diaphanous 1 (mDia1), of Cdc42 with a mutant mDial and of Rho1 from Entamoeba histolytica with formin1. We will describe the RhoC-mDia structure in detail and then discuss the differences in the Cdc42 and EhRho1 structures.

The fragment of mDial used in the crystallization included the Rho binding domain and the adjacent formin homology 3 (FH3) domain (Rose et al. 2005). The mDial protein was dimeric in the crystal and this was confirmed by gel filtration results. The dimer interface is extensive and hydrophobic suggesting that it would be maintained in the context of the full-length protein. The mDia1 protein is completely helical and includes three domains or subdomains: the GTPase binding domain, an armadillo repeat domain and a dimerization domain. All of the interactions with $\mathrm{RhoC}$ come from just one monomer and the dimer interface does not involve any helices that interact with the $\mathrm{G}$ protein.

Most of the interactions with the RhoC switch regions involve the second and third helices from the GTPase binding domain, which are thus designated as the N- and Cterminal helices of the helical pair in our classification system. Another helix in the GTPase binding domain, helix 1, serves to pack behind the two main interacting helices, stabilizing their orientation and fixing them into a competent GTPase binding fold (Figure 3.7). It contributes two residues to the interface with RhoC: Met94 and Asn95, which are involved in contacts with switch 2 residues $\mathrm{Arg} 68^{\mathrm{RhoC}}$ and Leu69 ${ }^{\mathrm{RhoC}}$. Switch 1 contacts only involve the helical pair: Val38 ${ }^{\mathrm{RhoC}}$ and Phe $39^{\mathrm{RhoC}}$ make hydrophobic contacts with Pro $103^{\mathrm{mDia}}$ and Met $115^{\mathrm{mDia}}$, while Glu $40^{\mathrm{RhoC}}$ forms a salt bridge with Lys $107^{\mathrm{mDia}}$. Within the interswitch, $\operatorname{Trp} 58^{\mathrm{RhoC}}$ (equivalent to position 56 in Ras) interacts with Lys $107^{\mathrm{mDia}}$. In switch $2, \mathrm{Gln} 63^{\mathrm{RhoC}}$ and Tyr66 ${ }^{\mathrm{RhoC}}$ form hydrogen bonds with Lys $100^{\mathrm{mDia}}$, while Le- $69^{\mathrm{RhoC}}$ and Leu $72^{\mathrm{RhoC}}$ form hydrophobic 
contacts with Met $115^{\mathrm{mDia}}$. The mainchain of Leu72 also forms a hydrogen bond with Gln $118^{\mathrm{mDia}}$. Apart from these contacts with the helices in the GTPase binding domain, RhoC also contacts residues within the armadillo repeat. These include switch 2 residues, Asp6 $7^{\text {RhoC }}$, which forms hydrogen bonds with Asn164/Asn165 ${ }^{\mathrm{mDia}}$ and $\operatorname{Arg} 68^{\text {RhoC }}$ which hydrogen bonds with Asn2 $17^{\mathrm{mDia}}$. Residues outside the switch regions also contact the armadillo domain: Glu102, His 105 and Phe106 in RhoC helix 3 and Lys133/Met134 in the RhoC 'insert helix' (see Figure 1.2) are involved in these interactions.

RhoC binds to mDial with a Kd of 6nM (Rose et al. 2005), and many of the residues used in the interaction are conserved between RhoA-C. Cdc42 and Rac1 do not bind to mDia but many of the interacting residues are conserved between the Rho proteins, particularly in the switch regions. Phe106 in RhoC was mutated His, the Cdc42 counterpart, and the affinity was reduced to $44 \mu \mathrm{M}$ (Rose et al. 2005), suggesting that residues outside the switch region are important determinants for specificity. When His 104 in Cdc42 (the equivalent residue) was mutated to Phe, the binding affinity for mDial was increased from $7.5 \mu \mathrm{M}$ to $100 \mathrm{nM}$ (Lammers et al. 2008).

The mDia1 related proteins mDia2 and mDia3 interact with $\mathrm{Cdc} 42$ and sequence analysis suggested that the promiscuity of the latter isoforms is due to a triple Asn motif within the armadillo repeats (Asn164-Asn165-Asn166), which contact several residues in RhoC. These were mutated to Thr-Ser-His, as in $\mathrm{mDia} 2 / 3$ and this mutant was found to bind more tightly to $\mathrm{Cdc} 42$, albeit with only 6-fold increased affinity (Lammers et al. 2008). The structure of the complex formed between 'mDia1-TSH' and Cdc42 was solved and found to form many interactions identical to those in the RhoC-mDial complex (Lammers et al. 2008). Most of switch 1 and all of switch 2 are conserved between RhoC and $\mathrm{Cdc} 42$, so that their interactions are also conserved. Within switch 1, Glu40 ${ }^{\mathrm{RhoC}}$ is replaced by Asp $38^{\mathrm{Cdc} 42}$ and the shorter sidechain of the Asp means that it no longer forms a salt bridge with Lys $107^{\mathrm{mDia}}$. This loss of a salt bridge may account for some of the reduced affinity for the Cdc42-mDia1-TSH complex $\left(K_{\mathrm{d}} 1.2 \mu \mathrm{M}\right)$. The interactions formed with the Thr-Ser-His residues are also different, since this motif is Asn-Asn-Asn in wild-type mDia1. The His103 residue in Cdc42 is contacted by His 166 from mDia-TSH, which would be the smaller Asn166 
sidechain in the mDial natural protein. The larger His166 sidechain can contact His 103, whereas Asn166 would leave a cavity. This may explain in part the increased affinity of the Thr-Ser-His mutant.

Interestingly, the Rho insert helix is not involved in the interactions between Cdc42 and mDia-TSH. The sequence in this region is not conserved amongst the Rho family proteins and instead the Lys-131 sidechain forms a salt bridge within the Cdc42 molecule. The insert region is absent in the EhRhol protein and thus there are no interactions in this region in the EhRho1-EhFormin1 complex (Bosch et al. 2012). Despite the lack of sequence conservation in the Entamoeba histolytica orthologues, the structure of the Eh complex is more less the same, although the N-terminal helix of the helical pair is shorter (a single turn) and the C-terminal helix is extended.

\subsection{Type E}

The final group of anti-parallel helical pair effectors contains only one example so far, which is Arl1 with Arfaptin2 (Figure 3.8). The interacting helices form a long, banana-shaped molecule whose orientation points them downwards from left to right in this viewpoint.

\subsubsection{Arl1-Arfaptin}

The Arfaptin helical pair is within a triple helical coiled coil, but only two of the helices form contacts with the Arl1 protein (Nakamura et al. 2012). The first helix in the Arfaptin molecule (helix 1) does not interact with Arl1 at all, but is packed behind the helical pair.

The Arfaptin helical pair is contained within a BAR domain, a protein domain that forms a homodimer that is capable of inducing membrane curvature. The membranebinding region of Arfaptin is on the concave face of the crescent-shaped molecule (Figure 3.9). Although the Arfaptin BAR domain is a dimer, each monomer only contacts a single Arfl molecule, which binds on the convex surface and thus will not prevent membrane association of the BAR domain.

Most switch 1 interactions are made by the C-terminal helix of the BAR domain helical pair: Gly50 ${ }^{\text {Arl1 }}$ and Phe $51^{\text {Arl1 }}$ contact Phe285 ${ }^{\text {BAR }}$, while Asn52 $2^{\text {Arl1 }}$ forms a 
hydrogen bond with Lys292 ${ }^{\text {BAR }}$. Val53 ${ }^{\text {Arl1 }}$ contacts the N-terminal helix at Thr $217^{\text {BAR }}$. Within the interswitch region, Thr55 $5^{\text {Arl } 1}$ hydrogen bonds with Thr $212^{\text {BAR }}$, while Gln64 ${ }^{\text {Arl } 1}$ contacts Lys $216^{\text {BAR }}$. The hydrophobic triad residue Trp66 ${ }^{\text {Arl1 }}$ contacts Asp $220^{\text {BAR }}$. Switch 2 contacts are only with the C-terminal helix of the BAR domain: Ile $74^{\text {Arl } 1}$ packs against Phe $285^{\text {BAR }}$ and Tyr77 ${ }^{\text {Arl1 }}$ against Il-281 ${ }^{\text {BAR }}$. Cys80 ${ }^{\text {Arl1 }}$ forms a hydrogen bond with Asp $378^{\mathrm{BAR}}$. The third residue in the hydrophobic triad, Tyr81 $1^{\mathrm{Arl} 1}$, contacts Asp $220^{\mathrm{BAR}}$ in the N-terminal helix and Lys282/Phe $285^{\mathrm{BAR}}$ in the C-terminal helix. Outside the switch regions, Glu1 $7^{\text {Arl1 }}$ and Arg19 ${ }^{\text {Arl1 }}$ are also involved in interactions, forming salt bridges with Lys $216^{\mathrm{BAR}}$ and Asp $220^{\mathrm{BAR}}$ respectively.

Mutations were made in Arl1 and tested for interaction with the Arfaptin protein qualitatively using GST-pulldown experiments. Arl1 mutants E17A, F51A ad G50T all appeared to bind to Arfaptin similarly to the wild-type protein. In contrast, Y81A and to a lesser extent W66A and R19A mutants reduced the binding. Hence, only two of the three residues in the hydrophobic triad are necessary for high affinity interactions. Furthermore, the Y81A mutant was unable to localize Arfaptin to the Golgi in HeLa cells (Nakamura et al. 2012).

Arfaptin has also been shown to bind to Rac1, although this interaction is not GTPdependent and so Arfaptin cannot be characterized as a Rac1 effector protein. Even though Rac1 and Arl1 are both G proteins, with a conserved structure and a certain amount of conservation at the sequence level, Rac1 binds to a different interface, the concave interface of the Arfaptin dimer (Tarricone et al. 2001) (Figure 3.8C). Like the Arl1-Arfaptin interaction, only two of the Arfaptin helices are involved in the interaction. In this case however it is the first and second Arfaptin helices and the Rac1-Arfaptin complex would fit into the Type B class of helical pair interactions. Even though the Rac1-Arfaptin interaction is not nucleotide dependent, the Rac1 regions that interact are confined to the switch regions and the adjacent residues, including the interswitch. The switches in the Rac1 -GMPPNP complex are in a GDPbound conformation, suggesting that the Rac1-Arfpatin interaction is incompatible with a GTP-bound Rac1 state. All but one of the Rac1 interactions involve just one of the Arfaptin monomers in the crescent-shaped dimer, but the complex is asymmetric. Only one Rac1 can be accommodated on the concave surface where it binds and 
interaction of a second Rac1 would be sterically hindered by the presence of the first one.

As Rac1 binds to the concave surface of the Arfaptin dimer, it is clear that its binding will inhibit membrane curvature by Arfaptin. Arll binding however leaves the membrane-binding face of Arfaptin free and thus as well as recruiting Arfaptin to target membrane would allow membrane deformation by the effector molecule. Modelling suggests that one of the two Arl1 molecules in the 2:2 complex with Arfaptin will clash with the single Rac1 and this seemed to be case: SPR results were consistent with formation of a 1:1:1 Arl1:Arfaptin:Rac1 complex (Nakamura et al. 2012).

\subsection{Class F}

The first class of effectors that bind using a parallel pair of $\alpha$-helices is that where the $\mathrm{N}$-termini of the helices point to the right hand side in the orientation in Figure 3.1, towards the switch regions of the $\mathrm{G}$ domain. This class includes representatives from the Ras, Rho, Arf and Rab subfamilies and displays the usual diversity in the nature of interactions that can be made with the small G protein (Figure 3.9). Within the Ras family the only example is that of the Ras exchange factor Sos in complex with Ras.GMPPNP at the 'catalytic site'. The helical pair binds across the face of the Ras protein and point down from left to right in this view. The Rho family effector that contacts using parallel helices is ROCK1, which binds using two very long $\alpha$-helices that form a parallel coiled-coil and only contact RhoA at their C-terminal end. There are three Arf effector structures that bind in this class. Firstly, JIP4 binds using a long parallel coiled-coil that contacts the Arf6 protein in the center. The $\gamma$-subunit of the COP1 coatomer and the $\beta 1$-subunit of the AP-1 adaptor are homologous and also bind to Arf1 using a shorter pair of parallel $\alpha$-helices. Interestingly, their orientation is very similar to the Ras-Sos interaction helices (Figure 3.9A, C). In particular, both of these pairs of helices are from the same effector monomer and are linked together by a third helix that fastens across their back face, stabilizing the parallel helical pair (see Figure 3.9A, C). The Rab family effectors that bind using parallel coiled-coils include the Rab11 family of interacting proteins, FIP2 and FIP3, which use a pair of long helices that bind to Rabs via their C-termini and are oriented pointing up from left to 
right in this view and engage one side of the Rab molecule (Figure 3.9E). The Rabaptin5 effector forms a similar structure but its interaction with Rab5 is very different. The helical pair is almost horizontal in this orientation and lies across the front face of the Rab molecule (Figure 3.9F). Finally, the VARP effector utilizes two short $\alpha$-helices to contact Rab32, which are similar in position and orientation to the ROCK1 helices that bind to RhoA, although the VARP helices are only three helical turns compared to the 17 turns found in ROCK1.

\subsubsection{H-Ras-Sos}

The H-Ras exchange factor Sos (son of sevenless) includes a catalytic Cdc25 domain and a Ras exchanger motif (REM) domain that appears to allosterically modulate the exchange activity of the Cdc25. Apart from the nucleotide-free Ras that is bound at the Cdc25 catalytic site, there is a second Ras molecule bound to the Sos molecule at a second, distal site (Margarit et al. 2003). This binding of the Ras switch mainly involves the Sos REM domain, although the Cdc 25 domain also makes contacts with Ras outside the switches. This immediately provides a potential mechanism for the positive feedback that would ensue from Ras engagement at this site.

The two $\alpha$-helices that form the parallel helical pair are at the C-terminus of the REM domain. They are connected by another long helix, which packs behind the helical pair (Figure 3.9A). This third helix makes no contacts with Ras but serves to connect the two interacting helices, forming hydrophobic contacts with the helical pair and providing a platform to present them in the correct orientation.

The switch regions of Ras interact exclusively with the parallel helical pair, making a number of polar contacts and very few hydrophobic interactions. In switch 1, Glu31/Asp33 ${ }^{\text {Ras }}$ form salt bridges with Arg $739^{\text {Sos }}$, Pro34 ${ }^{\text {Ras }}$ mainchain forms a hydrogen bond with Arg694 ${ }^{\text {Sos }}$, Ile36 ${ }^{\text {Ras }}$ interacts with Trp $729^{\text {Sos }}$, Glu37 $7^{\text {Ras }}$ hydrogen bonds to Arg688/Arg691 ${ }^{\text {Sos }}$ and Asp38 ${ }^{\text {Ras }}$ forms a salt bridge with His695 ${ }^{\text {Sos }}$. In switch 2, Gln61 ${ }^{\text {Ras }}$ hydrogen bonds to Lys $728^{\text {Sos }}$, Glu63 ${ }^{\text {Ras }}$ hydrogen bonds to the mainchain of Lys724/Ala725 $5^{\text {Sos }}$, Al-64/Met67 $7^{\text {Ras }}$ pack against Leu68 $7^{\text {Sos }}$ and Gln $70^{\text {Ras }}$ hydrogen bonds with $\operatorname{Arg} 688^{\text {Sos }}$. 
The interactions with the rest of the Sos molecule all involve the $\operatorname{Cdc} 25$ domain and can be divided into two areas of interaction. The first involves the base of the "helical hairpin' of the Cdc25 domain, which inserts into the Ras bound at the Sos active site, pushing switch 1 out of the way and enabling exchange (Boriack-Sjodin et al. 1998) (Figure 3.10). The N-terminus of Ras (Met1, Ile24 and Gln25) makes a mixture of hydrophobic and polar contacts with Sos residues at the base of the hairpin, while Ras residues in the interswitch region (Gln43 and Thr50) form hydrogen bonds with the same region of Sos. The other site of interaction with the Cdc 25 domain involves the long unstructured region that links the end of the REM domain and the beginning of the structured part of the Cdc25 domain. Polar contacts are made with this unstructured region with Asn26 and His 27 of Ras, just before switch 1, and around the Ras C-terminal helix (Arg149 and Glu153).

The consequences of Ras binding at the Sos allosteric site are a reorientation of the REM and Cdc25 domains, such that new hydrogen bonds are formed with switch 1 of the Ras bound at the catalytic site. The consequence of these interactions is that the exchange rate of Sos is increased up to 8-fold when active Ras is added to in vitro exchange reactions (Margarit et al. 2003).

\subsubsection{RhoA-ROCK1}

ROCK1 is a serine/threonine kinase that mediates the role of Rho proteins in smooth muscle contraction and cell migration. It contains a central large coiled-coil region, which includes the Rho binding domain. The structure of the ROCK1-RhoA complex showed that the Rho-binding domain dimerizes via a parallel coiled-coil made up of two long (65 residue) $\alpha$-helices (Dvorsky et al. 2004). Within the coiled-coil, only a relatively small region at the $\mathrm{C}$-terminus interacts with two RhoA proteins (Figure 3.9B). The interactions with RhoA are symmetric: one of the helices in the coiled-coil faces switch 1, while the other helix faces switch 2 and the other RhoA molecule in the tetramer makes identical interactions. In the description of the contacts below, the ROCK1 helix that faces switch 1 is called $X$, while the helix that faces switch 2 is called Y, so that they can be distinguished. 
The interactions in the RhoA-ROCK1 interface are a mixture of hydrophobic and polar contacts. In switch 1 , the contacts are mainly hydrophobic: Pro36 ${ }^{\text {Rho }}$ contacts Leu998 ${ }^{\text {ROCKX }}$, Val38 ${ }^{\text {Rho }}$ packs against Lys $1005 / \mathrm{Gln} 1001^{\text {ROCKX }}$ and Leu1006 ${ }^{\text {ROCKY }}$ and Phe $39^{\text {Rho }}$ contacts Lys $1005^{\text {ROCKX }}$ and Met $1010^{\text {ROCKY }}$. Gl- $40^{\text {Rho }}$ at the end of switch 1 then makes a salt bridge with Lys $1005^{\mathrm{ROCKX}}$. In switch 2 there are more polar contacts that in switch 1 but again the interactions involve both ROCK1 helices. Gln63 ${ }^{\text {Rho }}$ contacts Leu998 ${ }^{\text {ROCKX }}$, Asp65 ${ }^{\text {Rho }}$ forms a salt bridge with Lys999 ${ }^{\text {ROCKY }}$, Tyr66 ${ }^{\text {Rho }}$ forms hydrophobic interactions with Val1003 ${ }^{\text {ROCKY }}$ and Leu $998^{\text {ROCKX }}$ but also forms a hydrogen bond with the mainchain of Leu998. Arg $68^{\text {Rho }}$ hydrogen bonds with Asn $1004^{\text {ROCKY }}$. This is followed by two hydrophobic residues, Leu69 $9^{\text {Rho }}$ and Leu $72^{\text {Rho }}$, which contact Ala1007/Met1010 ROCKY . The only interaction outside the switch regions is Pro $75^{\text {Rho }}$, which packs against the methylene portion of the $\operatorname{Arg} 1012^{\text {ROCKY }}$ sidechain.

Mutations in RhoA that disrupt ROCK1 binding include F39A, F39L, E40L and E40W (Sahai et al. 1998). These residues are both within the interface described, so that the effects of their mutation can be readily explained from this structure. ROCK proteins bind to RhoA but not to Rac1 or Cdc42. The residues involved in the RhoAROCK1 interaction are identical in Cdc42 and Rac1, with the exception of Glu40, which forms a salt bridge with Lys1005 in ROCK1. This Glu is replaced by Asp38 in Cdc42 and Rac1 and presumably, as it is shorter, cannot form the salt bridge. It has also been suggested that the hydrophobic patch in RhoA comprising Pro36, Val38, Tyr66 and Leu72 also may contribute to the specificity since some of these residues are in different orientations in Cdc42 and Rac1 structures (Dvorsky et al. 2004). Such comparisons should be interpreted with caution however, since the switch regions are generally rather flexible in the free $G$ proteins and the positions of the sidechains are likely to move when complexes are formed.

\subsubsection{Arf1-coatomer and Arf1-AP1}

Activated Arf1 is localized to Golgi, where it recruits coat protein complexes (COPs) that coat the membrane surface and facilitate bud formation. COP1 vesicles are formed when seven COPs assemble on the Golgi, which self-assemble into a cage around the nascent bud and also interact with cargo molecules. COP1 coats are involved in retrograde transport from Golgi to ER. For transport between endosomes 
and the trans-Golgi network the vesicles utilize a clathrin coat combined with the AP1 adaptor complex (reviewed in (Mcmahon \& Mills 2004)). The COP1 coat combines a trimeric cage-forming $\alpha \beta^{\prime} \varepsilon$ complex with a tetrameric $\beta \delta / \gamma \zeta$ complex that binds to Arf1. In the clathrin-AP1 complex, clathrin forms the cage and the tetrameric $\gamma-\beta 1$ $\mu 1-\sigma 1$ complex binds to Arfl. The tetrameric Arf-binding complexes are related both in architecture and in their amino acid sequence. It is thought that the cytoplasmic forms of AP1 and COP are in a 'closed' conformation and that binding to membrane and activating molecules allows formation of the 'open' conformation, where the cargo binding sites are exposed.

The structure of Arfl with the minimal binding domains from the $\gamma \zeta-\mathrm{COP}$ subcomplex shows that this large assembly interacts with the Arf1 switch regions via a pair of $\alpha$-helices within the $\gamma$-COP subunit (Yu et al. 2012). The parallel helices are connected by a third helix that packs behind them, forming a platform to present the interacting helices. As discussed above, this architecture is similar to regions of Sos that contact the Ras switch regions (Section 3.5.1).

The majority of the interactions between Arf1 and COP are hydrophobic and most contacts involve switch 1 residues, with rather fewer in switch 2. The two helices in the helical pair encompass residues 62-79 ( $\alpha 4)$ and 101-115 ( $\alpha 6)$ of $\gamma$-COP and these make almost all of the interactions. Helix $\alpha 6$ and the loop between $\alpha 1$ and $\alpha 2$ of $\gamma$ COP, which together flank the interacting helices also make one or two contacts with Arf1 (Figure 3.11A). In switch 1, Ile46 ${ }^{\text {Arf }}$ contacts Ser107/Tyr143 ${ }^{\text {COP }}$, Ile49 ${ }^{\text {Arf }}$ interacts with Ile103/Thr106/Met136 ${ }^{\mathrm{COP}}$, Gly50 ${ }^{\text {Arf }}$ contacts Ile104/Ser107 ${ }^{\mathrm{COP}}$, Phe $51^{\text {Arf }}$ contacts Thr74/Ser103/Ile-104 ${ }^{\mathrm{COP}}$ and As-52 ${ }^{\mathrm{Arf}}$ contacts Ser107/Lys $111^{\mathrm{COP}}$. Within switch 2 there are only two interacting residues: Leu77 $7^{\text {Arf }}$, which contacts Asp $101^{\mathrm{COP}}$ and His $80^{\mathrm{Arf}}$, which contacts Phe $71^{\mathrm{COP}}$. The interswitch region makes more polar contacts: Glu54 ${ }^{\mathrm{Arf}}$ to Lys $111^{\mathrm{COP}}$, Thr6 $4^{\mathrm{Arf}}$ to $\mathrm{Gln} 78^{\mathrm{COP}}$ and $\operatorname{Tr}-66^{\mathrm{Arf}}$ to Lys $74^{\mathrm{COP}}$. The third residue in the hydrophobic triad, Tyr81, contacts Phe $71 /$ Thr $74^{\text {COP }}$. There are only two other interactions outside the switches and both are polar: Arg $19^{\mathrm{Arf}}$ to Lys $75^{\mathrm{COP}}$ and Asn $84^{\mathrm{Arf}}$ to Glu36 ${ }^{\mathrm{COP}}$. 
Arf1 mutations that knock down the binding of the coatomer were F31E, L77E and Y81E (Yu et al. 2012). These same mutations, as well as mutants in the $\gamma$-COP at the interface with Arf1, were used to confirm the observation that Arfl also binds to the $\beta \delta$-COP subcomplex. This allowed a model to be proposed for how Arf1 interactions may lead to formation of an open coatomer complex at the target membrane (Yu et al. 2012).

More insight into the mechanism of open complex formation came from the structure of the Arf1-AP1 adaptor complex structure (Ren et al. 2013). This structure included the AP1 heterotetramer $(\gamma-\beta 1-\mu 1-\sigma 1)$ in complex with Arf1 and although relatively low resolution $(7 \AA)$ it provides the first structural data on the entire AP1 core. The crystals contained one copy of Arf1 bound to the AP1 core and the Arf1 switch regions contacted the $\beta 1$ subunit of $\mathrm{AP} 1$, in a contact surface similar to the one described above between Arf1 and $\gamma$-COP (Figure 3.11B). A second binding site was also proposed on the AP1 $\gamma$ subunit, on the basis of crosslinking studies and the similarities with COP1 interactions. Mutational analysis with qualitative GSTpulldown assays suggested that $\gamma$ does bind to Arf1 in solution, albeit with a lower affinity than the Arf1- $\beta$ interaction (Ren et al. 2013).

The extra insight from the AP1 structural work came from two observations. Firstly, the AP1 tetramer was in the open conformation, even though it only contains Arf1 and AP1 and not other molecules such as cargo tails or phosphoinositides, which stabilize the open conformation of the related adaptor AP2 (Jackson et al. 2010; Jackson et al. 2012). Secondly, it was found that in the crystal lattice Arf1 bridges two AP1 tetramers (Ren et al. 2013), using the back face of Arf1 to contact a separate region of the $\gamma$ subunit (i.e. not at the predicted interface between the Arfl switches and $\gamma$ ). This interface involves hydrophobic residues in $\alpha 4, \beta 6$ and $\alpha 5$ of Arf1 and is smaller than the main interface involving the switches (Figure 3.11B). Mutations in the Arf1 residues that formed these contacts did not inhibit binding of AP1, suggesting that the interface is not important for recruitment of AP1 to membranes by Arf1. One of these mutants however was found to inhibit AP1 activation, as assessed by its ability to bind to immobilized cargo peptide. This led to a model where 2 Arf1 molecules recruit AP1 via binding to $\beta 1$ and $\gamma$ subunits using the Arf1 switch regions. 
The presence of other Arf1-AP1 complexes in the vicinity leads to high local concentrations and formation of the dimer that utilizes the back interface of Arf1 and a slightly different surface on the $\gamma$ subunit. This then leads to formation of the open, active state of AP1. The interaction between the switch region of Arf1 and $\gamma$ is weak and once it has been used for recruitment the contact here is broken during the formation of the open state.

\subsubsection{Arf6-JIP4}

The c-Jun N-terminal kinase interacting proteins, JIP3 and JIP4 are specific effectors for Arf6. They are scaffolding proteins that bind to Arf6, molecular motors and JNK/MAP kinase pathway proteins and are important for regulation of endosome traffic during cytokinesis. Like ROCK1, the JIP4 is a dimeric coiled-coil that in the crystal structure interacts symmetrically with two G proteins (Isabet et al. 2009). The heterotetramer was also observed in solution using analytical ultracentrifugation and under conditions of excess JIP4, a heterotrimer could be observed, suggesting that the Arf6 binding is not cooperative. In this case, Arf6 interacts with the center of the coiled-coil whose structure was solved, rather than one end, as was the case with RhoA-ROCK1. As the structure is symmetric we will only describe interactions with one Arf6 monomer (chain A in the PDB file) and will denote the two JIP4 monomers with superscripts $\mathrm{C}$ and $\mathrm{D}$.

The Arf6-JIP4 interactions are mainly hydrophobic within the switch regions, but involve several hydrogen bonds and salt bridges in the regions outside the switches. The interactions with the switch regions involve both helices C and D of the JIP4 dimer, while the interswitch region mainly interacts with monomer D. Hydrophobic interactions are formed with both helices in the JIP4 dimer, while the salt bridges and hydrogen bonds are only made with one of them ( $\mathrm{D}$ in our nomenclature). In switch 1, Phe $47^{\text {Arf6 }}$ contacts Leu $420^{\mathrm{C}}$ and Lys $423^{\mathrm{C}}$, while Val49 ${ }^{\text {Arf6 }}$ packs against Val424 ${ }^{\mathrm{D}}$. Within switch 2, Leu $73^{\text {Arf6 }}$ makes hydrophobic interactions with $\mathrm{Il}-415^{\mathrm{C}}$, Val416 ${ }^{\mathrm{C}}$ and Ala $412^{\mathrm{C}}$ and the His 76 sidechain contacts Ala $412^{\mathrm{C}} / \mathrm{Leu} 413^{\mathrm{C}}$ while its mainchain forms a hydrogen bond with Lys $417^{\mathrm{D}}$. There are therefore only two residues in each switch region that interact but in the interswitch region there are 4 residues that pack against JIP4, reflecting its importance in conformational changes in the Arf family. 
The interswitch contacts are generally hydrogen bonds and salt bridges, which are expected to be more specific than hydrophobic interactions. Thr $51^{\text {Arf6 }}$ forms a hydrogen bond with $\mathrm{Thr} 428^{\mathrm{D}}$, Lys $58^{\text {Arf6 }}$ forms a salt bridge with Asp $432^{\mathrm{D}}$, Asn60 forms a hydrogen bond with $\mathrm{Asp} 425^{\mathrm{D}}$ and Trp62, as well as its aromatic rings packing against Leu $420^{\mathrm{C}}, \mathrm{Ile} 421^{\mathrm{D}}$ and Val424 ${ }^{\mathrm{D}}$, forms a hydrogen bond with Asp $425^{\mathrm{D}}$. Two residues just after switch 2 also contact the JIP4: Tyr77, which forms hydrophobic interactions and Thr79, which makes hydrogen bonds with Asn $418^{\mathrm{D}}$ and Lys $417^{\mathrm{D}}$. The only other interaction is a salt bridge between $\operatorname{Arg} 15^{\operatorname{Arf6}}$ and Asp425 ${ }^{\mathrm{D}}$.

JIP4 binds to Arf6 with a $K_{\mathrm{d}}$ of $0.4 \mu \mathrm{M}$, but the closely related Arf1 binds around 20fold more weakly $\left(K_{\mathrm{d}} 10 \mu \mathrm{M}\right)$. Analysis of the residues in Arf6 that bind to JIP4 showed that only three of them are not conserved in Arf1: Lys58 becomes Se-62, Asn60 becomes Thr64 and Thr79 becomes Gln83. In addition, analysis of the structure showed that Thr53 in Arf6 is replaced by Glu57 in Arf1, which would be longer and negative and would clash with Asp432 of JIP4. Arf1 variants were generated and tested for binding to JIP4. The Arf1 mutant E57T/Q83T bound more tightly than wtArf1 but was still weaker than Arf6. The remaining two residues were also mutated and the quadruple mutant Arf1, E57T/S62K/T64N/Q83T bound to JIP4 with a $K_{\mathrm{d}}$ of $0.2 \mu \mathrm{M}$ i.e. as tightly as Arf6 (Isabet et al. 2009).

\subsubsection{Rab11-FIP2/3}

The FIP proteins all contain a conserved Rab-binding domain and have been divided into three classes based on their domain architecture within the rest of the proteins. The Class 1 FIPs, which include FIP2, regulate plasma membrane recycling, the Class II FIPs, including FIP3 (or Arfophilin-1), may be involved in cytokinesis and also can bind to Arf family G proteins via a separate interacting region. The Class III FIPs, which is only FIP1, does not contain any other domains. The structures of FIP2 and FIP3 with Ralb11 have been published (Jagoe et al. 2006; Shiba et al. 2006; Eathiraj et al. 2006) as well as the structure of FIP2 with Rab25 (also known as Rab11c) (Lall et al. 2013). As these structures are all essentially the same, we will describe the first of these (pdb code $2 \mathrm{GZH})$. 
The FIP2 Rab-binding domain is dimeric in its free form, which was established using analytical ultracentrifugation and dynamic light scattering (Jagoe et al. 2006) and in the crystal structure forms a 2:2 tetrameric complex with Rab11. Each FIP2 monomer contains one long $\alpha$-helix (residues 453-491), which is followed by a loop then a single turn of $3_{10}$ helix, so that the monomer is L-shaped. Inter-dimer contacts are formed by the long helix, which forms a coiled-coil, and the $3_{10}$ helix, which contacts the long helix on the other monomer (Figure 3.9E). The contacts formed between Rab11 and the FIP2 dimer are symmetric and in the discussion below we have used the superscripts $\mathrm{C}$ and $\mathrm{D}$ to denote the two monomers. The monomer designated as $\mathrm{C}$ is the one whose C-terminal $3_{10}$ helix comes close to the Rab11 molecule whose interactions are being discussed: it is this monomer that makes the majority of the contacts with Rab11.

In the complex Rab11 switch 1 is buried between the two long helices of the FIP2 dimer, forming contacts with both monomers: Lys $41^{\text {Rab11 }}$ forms a salt bridge on the edge of the interface with Glu $476^{\mathrm{D}}$, otherwise the contacts are generally hydrophobic: Ile $44^{\text {Rab11 }}$ with Tyr480 $/$ Ile $481^{\text {C }}$ and Leu $477^{\mathrm{D}}$, Gly45 ${ }^{\text {Rab11 }}$ with Leu $485^{\mathrm{C}} / \mathrm{Tyr} 480^{\mathrm{D}}$, Val46 ${ }^{\text {Rab11 }}$ with Leu485 $/$ Met489 $^{\mathrm{C}}$, Glu47 ${ }^{\text {Rab11 }}$ with Leu496 ${ }^{\mathrm{C}}$ mainchain and Val489 ${ }^{\mathrm{C}}$, Phe $48^{\text {Rab11 }}$ with $\operatorname{Arg} 497^{\mathrm{C}} / \mathrm{Val} 498^{\mathrm{C}}$. The interswitch region and switch 2 only form contacts with monomer $\mathrm{C}$ of the FIP2 dimer. $\operatorname{Arg} 74^{\text {Rab11 }}$ forms a salt bridge with Asp $482^{\mathrm{C}}$ but the remainder of the switch 2 interactions are also hydrophobic: Ala75 ${ }^{\text {Rab11 }}$ with Val486 $/$ Asn $483^{\mathrm{C}}$, Ile $76^{\text {Rab11 }}$ with Val486 $/$ Met $489^{\mathrm{C}} / \mathrm{Leu} 485^{\mathrm{C}}$ and Ala79 ${ }^{\text {Rab11 }}$ with Met489 . The interswitch region makes a single interaction, where $\mathrm{Thr} 50^{\mathrm{Rab11}}$ forma a hydrogen bond with $\mathrm{Arg} 497^{\mathrm{C}}$ and just after switch 2 is another polar contact, between $\operatorname{Arg} 82^{\text {Rab11 }}$ and Glu490 .

Most of the interactions between Rab11 and the FIP2 dimer involve the long $\alpha$-helix that comprises the majority of the FIP2, except for the switch 1 interactions with Leu496, Arg497 and Val498, which are the last residue of the $3_{10}$ helix and the first two of the extended region between this helix and the C-terminus. A FIP2 construct that terminates at Met489 still forms a coiled-coil but no longer associates with Rab11, underlining the importance of the interactions with the C-terminus (Wei et al. 2006). The hydrophobic triad residues in Rab11 are Phe48, Trp65 and Tyr80. Of 
these three, only Phe48 is involved in the interaction with FIP2. This triad is conserved in Rab proteins, most of which do not bind to FIP2 and perhaps the lack of the triad involvement in the interaction explains some of the specificity. Rather, the specificity for Rab11 is thought to reside in the combination of an unusual structure, which means that switch 2 is pushed away from switch 1 allowing the FIP2 helices to make closer contact with switch 1 and the presence of Lys-41 and Thr-50 which are only in Rab7 and Rab11. The switch 2 rearrangement upon FIP2 and FIP3 binding appears to be unique to Rab11: other Rabs thought to bind to their effectors with a 'lock and key' type interaction, where switch 2 is pre-formed in well-defined conformation.

In contrast, in the Rab11-FIP3 structures, the hydrophobic triad residues Trp65 and Tyr80 are involved in the interaction: both of them pack against Met746 in chain C. This Met is conserved in FIP2 (Met489) and makes hydrophobic interactions with Rab11. The Trp-65 sidechain points towards FIP2 and is buried in the complex, although it is not close enough to make direct interactions, but Tyr80 points towards the interior of the Rab11 G domain and is not near to FIP2 at all. This suggests that the structural rearrangements in Rab11 switch 2 are slightly different when bound to FIP2 and FIP3.

\subsubsection{Rab5-Rabaptin5}

Rab5 regulates early endosome fusion and one of the effectors that it uses is the multidomain protein Rabaptin5. Rabaptin5 includes a domain that interacts with Rabex, the Rab5 exchange factor, and it is thought that a positive feedback loop exists between them (Zhang, et al. 2014b). Rabaptin also binds to Rab4 via a different binding site and is therefore likely to be a bridge between the sequential Rab5/Rab4mediated pathways of endosome fusion and recycling.

Rabaptin5 forms a dimeric parallel coiled-coil in the crystal and its dimerization in solution was confirmed by crosslinking experiments (Zhu et al. 2004). The coiled-coil is composed of two long $\alpha$-helices (residues $802-837$ ), followed by a $\sim 180^{\circ}$ turn and then a shorter $\alpha$-helix of two turns whose axis almost parallel to that of the long helix (Figure 3.9F). The presence of a long helical coiled-coil followed by the short helix 
means that there is some superficial structural similarity to the FIP2/3 coiled coil, although the orientation of the long and short helices is different (Figure 3.9 E, F). Rabaptin5 also contacts the Rab molecule very differently, utilizing the long helix only for interactions with the $\mathrm{G}$ protein, while the short helix is only used for the dimerization interface.

The Rabaptin5 coiled-coil is positioned so that it is almost parallel with the $\beta 2-\beta 3$ hairpin of Rab5. It therefore makes very few contacts with switch 1 but more contacts with switch 2. Both of the helices of the coiled coil contact Rab5 and the two helices will be denoted $\mathrm{F}$ and $\mathrm{G}$ in the description below. The two switch 1 interactions are between Gly $54^{\mathrm{Rab} 5}$ and Phe $82^{\mathrm{G}}$ and between the hydrophobic triad Phe $57^{\mathrm{Rab} 5}$ and Val830 $/$ Glu $833^{\mathrm{F}}$. Within the interswitch there is a hydrogen bond formed between Thr59 $9^{\mathrm{Rab} 5}$ and Gln $837^{\mathrm{F}}$, while the hydrophobic triad Trp $74^{\mathrm{Rab} 5}$ contacts Val830 $/ \mathrm{G} \ln 826^{\mathrm{F}}$. Switch 2 forms a mixture of polar and nonpolar interactions: Arg81 ${ }^{\text {Rab5 }}$ hydrogen bonds to Gln $816^{\mathrm{G}}$, while Tyr82 ${ }^{\mathrm{Rab} 5}$ forms a hydrogen bond with Asp $820^{\mathrm{G}}$. These are followed by Leu $85^{\mathrm{Rab5}}$, which contacts Val817 $7^{\mathrm{G}} / \mathrm{Gln} 818^{\mathrm{F}} / \mathrm{Phe} 821^{\mathrm{G}} / \mathrm{Val} 822^{\mathrm{F}}$ and Met88 ${ }^{\mathrm{Rab} 5}$ which contacts $\operatorname{Arg} 819^{\mathrm{F}} /$ Val $822^{\mathrm{F}}$. The last residue in the hydrophobic triad, Ty-89, contacts $P h e 821^{\mathrm{G}} / \mathrm{Val} 822^{\mathrm{F}} / \mathrm{G} \ln 826^{\mathrm{F}}$. The coiled-coil therefore packs so that similar numbers of interactions are made by each helix.

Mutants in Rab5 that reduced or abrogated the interaction with Rabaptin5 were: F57A, W74A, Y82A and Y89A. Hence, the contacts involving the hydrophobic triad are important for the binding affinity. Rabaptin5 binds only to Rab5 and comparison with other Rabs shows that the only residues that are not conserved in all Rabs are Thr59, Tyr82 and Met88. The combination of these three residues may therefore be responsible for the selectivity.

\subsubsection{Rab32-VARP}

Rab32 is an endosomal Rab protein whose effectors include VARP (for VPS9-domain ankyrin repeat protein). VARP is a Rab21 exchange factor (Zhang et al. 2006), which also includes two ankyrin repeat domains. VARP also binds to VAMP7, an RSNARE, inhibiting VAMP7's ability to form SNARE complexes and complete 
membrane fusion (Schaefer et al. 2012), thus implicating VARP is endosome dynamics.

VARP binds to both Rab32 and Rab38 via its first ankyrin repeat domain, which contains 5 ankyrin repeats, each of which comprises two parallel $\alpha$-helices, $\alpha \mathrm{A}$ and $\alpha \mathrm{B}$ (Hesketh et al. 2014). The adjacent ankyrin repeats are connected by relatively long loops so that the $\alpha \mathrm{B}$ helices form the convex face of a curved platform with the long loops forming the concave face and the $\alpha \mathrm{A}$ helices sandwiched between them (Figure 3.12A). The $\alpha \mathrm{B}$ helices from ankyrin repeats 2 and 3 form a parallel helical pair that interacts with the Rab32 protein in an orientation that places them in class F. The bulk of the interactions with Rab32 are formed with these helices, which encompass VARP residues 509-518 and 541-552. Both of the VARP helices contact switch 1, the interswitch and switch 2. In switch 1, Asp61 Rab32 forms a salt bridge with Lys546 ${ }^{\text {VARP }}$ and Phe62 $2^{\text {Rab32 }}$ packs against Tyr550 VARP , while in switch 2 Va194 ${ }^{\text {Rab32 }}$ packs against Leu514/His517 ${ }^{\text {VARP }}$. In addition, the switch 2 residue $\operatorname{Arg} 93^{\text {Rab32 }}$ forms a salt bridge with Asp480 VARP, which is in the $\alpha \mathrm{B}$ helix from ankyrin repeat 1 .

In the X-ray derived structure it was observed that the VARP molecule contacts a second Rab32, so that a heterotetramer is formed (Figure 3.12B) and the presence of the tetramer in solution was confirmed by analytical ultracentrifugation (Hesketh et al. 2014). The second interface is formed by the N-terminus of VARP and its first ankyrin repeat, which contact residues in both switch 1 and switch 2 . These contacts are mainly salt bridges and hydrogen bonds: in switch 1 , His $53^{\text {Rab32 }}$ to Arg462 VARP, Tyr54 ${ }^{\text {Rab32 }}$ to Asp460 ${ }^{\text {VARP }}$, Arg55 $5^{\text {Rab32 }}$ to Asp460 ${ }^{\text {VARP }}$; in switch 2, Glu86 ${ }^{\text {Rab32 }}$ to Gln $475^{\text {VARP }}$ and Asn90 Rab32 to Ser $477^{\text {VARP }}$. In contrast, there are very few contacts between the VARP domains and these are unlikely to drive the dimerization.

The second Rab32-VARP interface is considered to be secondary, although its buried surface area is $1460 \AA^{2}$, not much smaller than the primary interface, which buries $1530 \AA^{2}$. The VARP fragment used binds to Rab32 with a $K_{\mathrm{D}}$ of $2.5 \mu \mathrm{M}$ (Hesketh et al. 2014) but the Rab32 M91S, R93S double mutant, which removes one of the salt bridges in the primary interface binds so weakly that its interaction is not detectable 
by isothermal calorimetry. This suggests that the second interface is not sufficient to drive the interaction between VARP and Rab32.

\subsection{Type $G$}

The other class of parallel coiled-coil effectors, that where the C-terminus is on the right of the molecule in the scheme in Figure 3.1 only contains two members, but they are both effectors bound to Rab6, the Golgin GCC185 and the Rab6-interacting protein 1 (Rab6IP1). The orientation of the helical pairs are more less the same in the two structures (Figure 3.13), although the helices are different lengths in the Rab6IP1 structure and they come from the same R6IP1 molecule, whereas the helices are formed by a homodimer in the Golgin structure and therefore are the same length.

\subsubsection{Rab6-Golgin GCC185}

GCC185 is a coiled-coil protein that is involved in receiving vesicles from late endosomes at the Golgi. It has been shown that GCC185 is recruited to Golgi by interacting with Rab6 and Arl1 small G proteins. The structure of Rab6 in complex with the GCC185 Rab-binding domain was solved and a model proposed for how Rab6 binding promotes the interaction of GCC185 with Arl1 via its adjacent GRIP domain (Burguete et al. 2008).

Like several of the parallel coiled-coils, GCC185 is formed by a dimer of $\alpha$-helices from two different monomers, which interact symmetrically with two Rab6 molecules (Burguete et al. 2008). Gel filtration and light scattering showed that the GCC185 Rab-binding domain is dimeric in solution. Each helix in the dimer interacts with Rab6 and in the description below the monomers will be denoted D and E. In general, monomer D interacts with switch 1 and monomer E with switch 2.

The interface between GCC185 and Rab6 contains more polar contacts than many of the other Rab effector complexes, which often involve hydrophobic contacts with the conserved hydrophobic triad. In GCC185-Rab6 even the hydrophobic residues in the interface often pack against the hydrocarbon portion of polar sidechains. At the Nterminus of Rab6, Lys15 forms a hydrogen bond with $\operatorname{Thr} 1585^{\mathrm{E}}$. Within switch 1, Gln42 ${ }^{\text {Rab6 }}$ hydrogen bonds with Arg $1601^{\mathrm{D}}$, Ile46 packs against Lys $1597^{\mathrm{D}} / \mathrm{Ile} 1600^{\mathrm{D}}$, 
Asp $49^{\text {Rab6 }}$ forms a salt bridge with Lys $1597^{\mathrm{D}}$ and Phe $50^{\mathrm{Rab} 6}$ contacts Met1590 ${ }^{\mathrm{D}}$.

Within the interswitch region only the hydrophobic triad residue Trp67 ${ }^{\text {Rab6 }}$ makes contacts with Thr1585 ${ }^{\mathrm{E}}$. In switch 2, Arg $75^{\mathrm{Rab6}}$ forms a salt bridge with Glu1604 ${ }^{\mathrm{D}}$, Phe $75^{\text {Rab6 }}$ contacts Glu1599 ${ }^{\mathrm{E}}$, Ser77 ${ }^{\text {Rab6 }}$ contacts Leu1595 ${ }^{\mathrm{E}}$, Leu78 ${ }^{\text {Rab6 }}$ contacts Leu $1595^{\mathrm{E}} / \mathrm{G} \ln 1592^{\mathrm{E}}$ and Ser81 ${ }^{\mathrm{Rab} 6}$ forms a hydrogen bond with Glu1591 ${ }^{\mathrm{E}}$. The third residue in the triad, Tyr82 $2^{\mathrm{Rab} 6}$, contacts Ile- $1588^{\mathrm{E}}$ and hydrogen bonds with $\mathrm{G} \ln 1592^{\mathrm{E}}$.

As GCC185 also contains a GRIP domain adjacent to the Rab6 binding domain, the effect of Rab6 binding on Arl1 interaction was tested. It was found that while Arl1 binding to the GRIP domain alone was rather weak, when the Rab6 binding domain was included, Rab6 interaction enhanced the Arl1 binding (Burguete et al. 2008). The authors concluded that this occurs because Rab6 stabilizes the GCC185 dimer, providing a stable GRIP dimer that is competent for Arl1 binding.

\subsubsection{Rab6-Rab6IP1}

Another Rab6 effector that is involved in Golgi-endosome transport is Rab6IP1. This effector includes two adjacent domains towards its C-terminus, a RUN domain and a PLAT domain (Figure 3.14). The RUN domain is involved in binding to Rab proteins, while the PLAT domain is often found in membrane associated proteins and may therefore be involved in protein-lipid interactions. The Rab6 binding region is within these domains and although in the X-ray structure all the interactions with Rab6 involve the RUN domain it does not bind tightly in isolation (Recacha et al. 2009).

The RUN domain forms a helical bundle containing $8 \alpha$-helices that are arranged antiparallel or perpendicular to their sequential neighbours. The PLAT domain forms a $\beta$-sheet sandwich and is tightly associated with the RUN domain via hydrophobic interactions to form a rigid domain pair. Only two helices in the RUN domain are involved in the interaction with Rab6 and these are the long, N-terminal helix (H1) and the last helix in the domain, H8, which are parallel. These encompass residues 713-751 and 899-915.

Both of the helices in the RUN domain interact with both switch regions of Rab6. The interaction has a hydrophobic centre surrounded by polar and charge interactions. At 
the N-terminus of Rab6, Lys13 forms a salt bridge with Asp901 ${ }^{\text {RUN }}$. In switch 1 there are mostly hydrophobic contacts, between Ile $46^{\text {Rab6 }}$ and Lys739/Leu742 ${ }^{\text {RUN }}$, Ile $48^{\text {Rab6 }}$ and His $909^{\text {RUN }}$ and Phe $50^{\text {Rab6 }}$ and Gln905/His900 ${ }^{\text {RUN }}$ although Asp49 ${ }^{\text {Rab6 }}$ forms salt bridges with Arg735/Lys739 ${ }^{\text {RUN }}$. Within the interswitch region Gln65 $5^{\text {Rab6 }}$ forms a hydrogen bond with Gln905 ${ }^{\text {RUN }}$ and the conserved Trp6 $7^{\text {Rab6 }}$ packs against Tyr908 ${ }^{\text {RUN }}$. Switch 2 contacts are also mostly hydrophobic with a single salt bridge, which is between $\operatorname{Arg} 74^{\text {Rab6 }}$ and Glu $749^{\text {RUN }}$. The other contacts are Leu $72^{\text {Rab6 }}$ to Met746 ${ }^{\text {RUN }}$, Phe $75^{\text {Rab6 }}$ to Leu742/Ala915 $5^{\text {RUN }}$ and Leu $78^{\text {Rab6 }}$ to Leu911 ${ }^{\text {RUN }}$. The third conserved hydrophobic residue, Tyr82 ${ }^{\text {Rab6 }}$ packs against Tyr $908^{\text {RUN }}$, along with Arg84 ${ }^{\text {Rab6 }}$, which forms a hydrogen bond with the same RUN domain Tyr.

The only Rab6 residue that interacts with the RUN domain and that is unique to Rab6 is Lys13, which is not conserved in the other Rab proteins. Although this forms a salt bridge it is unlikely to be sufficient for discrimination between the different Rabs.

Discrimination between the Rab proteins may reside in the fine details of the structure and dynamics of the switch regions and how they are presented, rather than the absolute sequence.

\subsection{General remarks}

The helical pair is the most common type of small $\mathrm{G}$ protein-effector interaction. The reasons for the popularity of this mode of interaction may be due to the dynamic nature of the switch regions and the amenity of helices to allosteric rearrangements. The formation of intermolecular $\beta$-sheets involves two more rigid surfaces coming together and is likely to be less accommodating to changes in sequence.

It is clear that amongst the helical pairs certain types are more common. Hence Types $\mathrm{B}$ and $\mathrm{E}$ have only one member each, Type $\mathrm{C}$ has only 2 members, while Type $\mathrm{D}$ has 11. It would seem therefore that the interhelical loop is more likely to be away from the switch regions, closer to the interswitch $\beta$-strands and $\beta 1$ of the $\mathrm{G}$ domain (see Figure 3.1), as in Types C and D. The positioning of the interhelical loop, which may in itself be flexible, against switch 2, which may also be dynamic, may not allow sufficiently tight contacts to be made to ensure specificity. Type D is more common than Type $\mathrm{C}$, which implies that forming anti-parallel helix-helix interactions with the 
switch 2 helix $(\alpha 2)$ is more stable than the parallel interactions that would ensue in Type C. There are slightly more examples of anti-parallel helical pairs than parallel. Again there is a definite preference for Type F over Type G. In this case, the preferred option has the helices parallel to the switch $2 \alpha$-helix rather than anti-parallel.

\section{Alternative modes of interacting}

The effector complexes that we will discuss do not fall into the categories described above i.e. they do not form an intermolecular $\beta$-sheet or interact with the switch regions of the $\mathrm{G}$ domain using a helical pair. We have divided them based on their small $\mathrm{G}$ protein binding partners and include effectors that interact with the Rho, Arf, Rab and Ran subfamilies.

\subsection{Rho subfamily binding proteins.}

\subsection{1 p67 $^{\text {phox }}$-'Rac1}

NADPH oxidase enzymes in phagocytes are used for generating a superoxide anion $\left(\mathrm{O}_{2}{ }^{-}\right)$from NADPH, which can be further processed to produce reactive oxygen species. Together, the reactive oxygen species and superoxide are used for defense against microbial infection. The NADPH oxidase complex is formed by two membrane proteins, gp91 ${ }^{\text {phox }}$ (the catalytic core) and $\mathrm{p} 22^{\text {phox }}$, which together form cytochrome $\mathrm{b}_{558}$, three cytosolic proteins, $\mathrm{p} 47^{\text {phox }}, \mathrm{p} 40^{\text {phox }}$, and $\mathrm{p} 67^{\text {phox }}$ and a Rac small G protein (reviewed in (Bae et al. 2011)).

Rac binding to $\mathrm{p} 67^{\text {phox }}$ is essential for activation of the NADPH oxidase enzyme and the region of $\mathrm{p} 67^{\text {phox }}$ that interacts with Rac has been delineated to 200 residues at the $\mathrm{N}$-terminus of $\mathrm{p} 67$. This fragment forms four tetratricopeptide repeats (TPR), each of which comprises two antiparallel $\alpha$-helices, and binds to Racl with $K_{\mathrm{d}}$ around $2 \mu \mathrm{M}$ (Lapouge et al. 2000). After the fourth TPR repeat (TPR4) there is an extra $\alpha$-helix that packs against the second helix of TPR4. Overall, the nine helices form a distorted crescent, with the first helix (A) of each TPR and the ninth helix on the concave face. The last helix is followed by an extended C-terminal segment of almost 20 residues that packs against the inside of the crescent, making contacts with the A-helices of all the TPR motifs. Between the third and fourth TPR motifs is an insertion of 20 amino acids that forms a short anti-parallel $\beta$-sheet and a single turn $3_{10}$ helix. 
The major interactions with Rac1 involve the $\beta$-hairpin insertion and loops between TPR 1 and TPR 2 and between TPR2 and TPR3. The region of Rac1 that interacts with p67 mainly involves the N-terminal helix and residues at the start of switch 1.

Otherwise, unusually, the switch regions show very few interactions and switch 2 does not interact at all. The interaction interface is almost completely polar, with few hydrophobic contacts between the two proteins. N-terminal to switch 1 , Ser22 $2^{\text {Rac }}$ forms a hydrogen bond with $\operatorname{Arg} 102^{\mathrm{p} 67}$ and Asn26 $6^{\mathrm{Rac}}$ hydrogen bonds to Asn $104^{\mathrm{p} 67}$. In switch 1, Phe $28^{\text {Rac }}$ packs along the ring of His69 $9^{\mathrm{p} 67}$, Gly $30^{\mathrm{Rac}}$ mainchain hydrogen bonds to Asp $67^{\mathrm{p} 67}$ and Glu31 ${ }^{\text {Rac }}$ hydrogen bonds to Ser8 $7^{\mathrm{p} 67}$. The only other contacts are with residues in the loop preceding the final $\alpha$-helix in Rac1: Leu160 Rac1 mainchain hydrogen bonds to $\operatorname{Arg} 102^{\mathrm{p} 67}$ and Gln $162^{\mathrm{Rac} 1}$ forma a hydrogen bond with Asn $104^{\mathrm{p} 67}$.

The $\mathrm{p} 67^{\text {phox }}$ protein does not bind to Cdc42, despite its similarity to Rac1. The only residues that are different in the two $G$ proteins and that interact or are close to the interaction are Ala27 and Gly30, which are Lys and Ser respectively in Cdc42. Although Ala $27^{\text {Rac }}$ does not interact directly, its substitution with the larger, charged Lys sidechain may lead to clashes with $\mathrm{p} 67^{\text {phox }}$. The Rac A27K/G30S mutant cannot bind to the $\mathrm{p} 67^{\text {phox }}$ fragment any more, while a Cdc42 L27A/S30G mutant is able to bind with a $K_{\mathrm{d}}$ of $6 \mu \mathrm{M}$ (Lapouge et al. 2000). This suggests that these residues are at least partially responsible for the specificity.

\subsubsection{Rac1/Rnd1-plexin interactions}

The plexins are a family of sempahorin receptors that are involved in axon guidance and cell migration. Their intracellular regions interact directly with both Rho and Ras family small G proteins: they contain a RasGAP domain, into which is inserted a Rho family binding domain within a surface-exposed loop. The extracellular regions of plexins bind to dimeric semaphorins, such that ligand binding induces plexin dimerization. Ligand and Rac synergistically activate the RasGAP domain when they are bound to the extracellular and intracellular portions of plexins respectively (see (Siebold \& Jones 2013) and (Hota \& Buck 2012) for reviews). There are structures available for the Rho binding domain of plexinA2 in complex with Rnd1 (Wang et al. 
2011), of plexinB1 with Rac1 (Bell et al. 2011) and of plexin A1 with Rac1 (Wang et al. 2012).

The Rac binding domain of plexins is within the GAP domain, forming an insertion into the canonical RasGAP fold. The structures of isolated Rac/Rnd binding domains RBDs of plexin B1 (Tong et al. 2007), plexin A4A (pdb 4E74), plexin B2 (pdb 4E71), plexin C1 and plexin D1 (Wang et al. 2011) have been solved and showed that the (RBD) forms a ubiquitin-like fold. We will describe the structure of the plexinB1 RasGAP and RBD solved in complex with Rac1 (Bell et al. 2011), but all of the structures solved are broadly similar in the interaction between the $G$ protein and the RBD.. Unlike Ras effectors such as Raf, the plexin RBD does not interact with Rac1 via an intermolecular $\beta$-sheet with strand $\beta 2$ of the G protein (Figure 4.2A). Instead, a mixture of loops, strand and some helix in the plexinB1 interact with Rac1, providing a hydrophobic surface to bury residues in the both switch regions. In switch 1, Phe $37^{\text {Rac }}$ contacts Trp1807/Leu1815 ${ }^{\text {Plex }}$, while in switch 2 Leu6 $7^{\text {Rac }}$ contacts Leu $1815^{\text {Plex }}$ and Leu70 ${ }^{\text {Rac }}$ contacts Tyr1839/Leu1815/Thr1823 ${ }^{\text {Plex }}$. In addition, there are hydrogen bonds formed with the switch regions: Asp3 $8^{\text {Rac }}$ to Val1811 ${ }^{\text {Plex }}$ mainchain, Asn39 $9^{\text {Rac }}$ to Gly $1813^{\text {Plex }}$ mainchain, Asp63 $3^{\text {Rac }}$ to Lys $1840^{\text {Plex }}$ and Arg66 $6^{\text {Rac }}$ to Glu $1825^{\text {Plex }}$. There are no residues outside the switch regions involved in these interactions and the few residues involved as well as the relatively small buried surface area account for the relatively low affinity of the interaction (around $20 \mu \mathrm{M}$ $\mathrm{Kd}$ ) (Bell et al. 2011). This affinity is broadly similar to those observed for plexin B1 with Rac1 and Rnd1 (Tong et al. 2009).

The structures of Rac1 and Rnd1 in the plexin complexes do not explain the effects of $\mathrm{G}$ protein binding on the GAP activity of plexins, since there are no contacts between Rac and the GAP domain at all (Figure 4.2A). This is in agreement with in vitro GAP assays that show that Rac1 does not stimulate the RasGAP activity of plexin cytoplasmic domains. The same group however showed that artificial dimerization of the plexin cytoplasmic regions stimulated the GAP activity (Wang et al. 2012).

The crystal structures of three different plexin-G protein complexes have suggested ways in which the intracellular domains of plexin may form dimers or higher order

URL: http:/mc.manuscriptcentral.com/58mg Email: pfeffer@biochem.wisc.edu 
structures. The plexin B1 isolated RBD, both alone (Tong et al. 2007) and in complex with Rnd1 (Wang et al. 2011) forms a dimer, utilizing an intermolecular $\beta$-sheet (Figure 4.2B). The residues involved in this $\beta$-sheet are, however, involved in interactions with the RasGAP domain in longer constructs of plexins. The plexin A1 full cytoplasmic domain in complex with Rac1 was also a dimer, but in this case one of the plexin monomers was bound to Racl and the other was empty (Figure 4.2C). Furthermore, the dimerization interface utilized by the plexin A1 was not the same as in the plexin B1 RBD, rather the two plexin molecules were arranged in a head-to-tail fashion, with the GAP domains making interactions with each other and with the RBD on the opposite molecule. In the low resolution structure of the full cytoplasmic region of plexinB1 with Rac1, the asymmetric unit contains three copies each of Rac1 and plexinB1, which are not due to crystallographic symmetry, although there is no evidence for the existence of a heterohexamer in solution (Bell et al. 2011). The hexamer may only be able form in the high concentrations that can be attained at the plasma membrane. The existence of the hexamer suggests that Rac1 can interact with plexinB1 via a second interface that is distinct from the interface involving the switch regions described above (Figure 4.2D). This interface has a smaller surface area than the first and involves interactions between Rac1 residues Ile21, Thr25, Ala27 just before switch 1, Phe28 and Glu31 at the N-terminus of the switch, and contacts in the loop before the last $\alpha$-helix $\square \square \square \square \square 160$ and Gln162). Interestingly this interaction surface overlaps that between Rac1 and p67 ${ }^{\text {phox }}$ (See 4.1.1 and Table 1) suggesting that this region may be a Rac-specific interaction surface. The plexin molecules also interact, although with a small buried surface, suggesting that neither the plexinplexin nor the plexin-Rac1 second contact would be stable alone. Mutation of plexinB 1 residues in the second contact site prevented the activation of the GAP in a cell-based functional assay, suggesting that this contact is important for the activity of plexinB1 (Bell et al. 2011).

Taken together, the basis for plexin regulation by Rho family G proteins has not yet been elucidated, although several possibilities are suggested by the structures that have been solved. It is also possible that different mechanisms will prevail in the different plexin families. 


\subsection{Arf subfamily binding proteins}

\subsubsection{Arl2-BART}

BART is a small protein that was identified as an effector of the Arf-like protein Arl2. The function of BART is not clear, although it has been found in the mitochondrial intermembrane space where it binds adenine nucleotide transporter (Sharer et al. 2002) and in the nucleus where it may interact with STAT3 (Muromoto et al. 2008) and has been implicated in cytokinesis in T. brucei (Price et al. 2010).

BART forms a helical bundle comprising $6 \alpha$-helices in a unique topology with helices $\alpha 1, \alpha 2$ and $\alpha 3$ more or less parallel and helices $\alpha 4, \alpha 5$ and $\alpha 6$ close to perpendicular to the first three helices (Bailey et al. 2009). In the Arl2 complex there is a rearrangement of the interhelix orientations to accommodate Arl2 binding (Zhang et al. 2009). There are two main regions of Arl2 that interact with BART. The first involves the usual set of nucleotide-sensitive residues and primarily involves switch 1 and the interswitch residues (Figure 4.3A). The contacts are a mixture of hydrophobic and polar: in helix $\alpha 1$, Lys $34^{\mathrm{Arl}}$ forms a salt bridge with Glu5 $7^{\mathrm{BART}}$, Thr5 $7^{\mathrm{Arl}}$ is packed alongside Glu57 ${ }^{\mathrm{BART}}$, Gly49 ${ }^{\mathrm{Arl}}$ against Leu60 ${ }^{\mathrm{BART}}$ and Phe50/Ile52 $2^{\mathrm{Arl}}$ contact Phe $118^{\text {BART }}$. In the interswitch region Lys $53^{\text {Arl }}$ forms a salt bridge with Glu $56^{\text {BART }}$. The contacts in switch 2 involve Leu $73^{\text {Arl }}$ packing against Leu60 ${ }^{\text {BART }}$ and Tyr80 ${ }^{\text {BART }}$, which also contacts Leu $60^{\text {BART }}$ as well as forming a hydrogen bond with Thr63 ${ }^{\text {BART }}$.

As well as the described contacts within the $\mathrm{G}$ domain, BART contacts the $\mathrm{N}$-terminal $\alpha$-helix of Arl2, which is also nucleotide-sensitive (Figure 4.3B). The N-terminal helix is a feature of the Arf family and is usually myristoylated, allowing membrane interaction in the GTP-bound form (see Figure 1.2). Arl2 is not myristoylated, so that its N-terminal helix is free to interact with effectors. The helix is relatively hydrophobic, so that it is buried in a groove made by the BART helical bundle and contacts residues in BART helices 3, 4 and 5. The contacts involve Leu3, Leu4, Ile6, Leu7 and Met10 of Arl2, which form hydrophobic contacts with BART, although there are also hydrogen bonds formed by Lys $8^{\mathrm{Arl}}$ and Lys $11^{\mathrm{Arl}}$.

The importance of the two contact areas was validated using mutations and qualitative GST-pulldown assays (Zhang et al. 2009). Deletion of residues 2-4 of Arl2, or 
mutation of the leucines at position 3, 4, or 7 to Asp abrogated or severely reduced the binding to BART. The interactions with the switch regions are also important, since the F50A mutant (switch 1) also failed to bind, as did the switch 2 mutant Y80A. The two interfaces used by Arl2 are sufficient to give a high affinity interaction, which has a 30-40 nM Kd (Bailey et al. 2009).

\subsubsection{Arf6-CTA1}

The Arf proteins are so named because they can act as "ADP-ribosylation factors", activators for toxins that lead to transfer of ADP-ribose units from $\mathrm{NAD}^{+}$to subunits of heterotrimeric $\mathrm{G}$ proteins, often with disastrous consequences for the organism.

Cholera toxin and the related enterotoxin are produced by pathogenic bacteria and in both cases the enzyme that performs the ADP-ribosylation is formed by cleavage of the toxin A subunit into the A2 domain and the catalytic A1 domain, which interacts with Arf proteins (De Haan \& Hirst 2004). The A1 enzyme catalyzes transfer of ADP-ribose to its target protein, in a reaction that is allosterically activated by Arf proteins. The cholera toxin A1 (CTA1) is insoluble in isolation but its structure was solved in complex with Arf6 (O'Neal et al. 2005). As the CTA1 is selective for GTPbound Arf proteins, it behaves as an effector protein, albeit one produced by the pathogenic bacterium rather than by the host cell.

The CTA1 protein forms a mixed $\alpha$ and $\beta$ structure, which is not homologous to any Arf effectors, comprising a twisted, 7-stranded anti-parallel $\beta$-sheet, surrounded by loops and 9 short $\alpha$-helices (Figure 4.4). The interaction with Arf6 involves the switch regions and the interswitch of the G protein, utilizing similar residues to a normal cellular effector. Much of the interface is hydrophobic, which explains why the free CTA1 protein is not soluble. Rather, it is likely that the CTA1 is passed from CTA2, which it binds in the context of the full toxin protein, onto Arf6 where it can be activated. The hydrophobic interactions with switch 1 include Ile $42^{\text {Arf6 }}$ to Tyr30 ${ }^{\mathrm{CTA} 1}$, Val45 ${ }^{\text {Arf6 }}$ to Met37/L116 ${ }^{\mathrm{CTA} 1}$, Phe47 ${ }^{\text {Arf6 }}$ to Asn93/Pro120/Tyr150 ${ }^{\mathrm{CTA} 1}$ and Val49 ${ }^{\text {Arf6 }}$ to Tyr $149^{\text {CTA1 }}$. The conserved Trp ${ }^{\text {Arf6 }}$ in the interswitch region contacts Tyr149/Leu153 ${ }^{\mathrm{CTA} 1}$ and in switch 2 Leu73 ${ }^{\mathrm{Arf6}}$ contacts Phe95/Ala156 ${ }^{\mathrm{CTA} 1}$ while His $76^{\text {Arf6 }}$ contacts Pro92/Pro157/Asp160 ${ }^{\text {CTA1 }}$. The third triad residue Tyr77 ${ }^{\text {Arf6 }}$

URL: http:/mc.manuscriptcentral.com/66mg Email: pfeffer@biochem.wisc.edu 
contacts Pro92 ${ }^{\mathrm{CTA} 1}$. There are also some polar interactions in the interface between the proteins: Glu13 and Arg15 in Arf6 with Arg148 and Asn152 from CTA1.

The CTA1 structure has also been solved in the holotoxin complex, i.e. in association with the cholera B chain and the CTA2 domain (Zhang et al. 1995; O'Neal et al. 2004). The CTA1 structure is the same in both complexes, except for the activation loop of CTA1, which adopts an $\alpha$-helix in the Arf6-bound complex and is unstructured in the holotoxin complex. This loop is close to the NAD+ binding site and is thought to be important for the activation of the CTA1 ADP-ribosyl transferase activity. The face of CTA1 that contacts Arf6 is the same as that which contacts CTA2 in the holotoxin complex, suggesting that both binding partners stabilize the CTA1 protein by masking the hydrophobic surface residues. More recently, it has been found that disordered CTA1 in the cell is folded by lipid rafts prior to activation by Arf6 (Banerjee et al. 2014).

\subsection{Rab subfamily binding proteins}

\subsubsection{Rab5-EEA1}

As mentioned previously, Rab5 is responsible for regulating endosomes via a number of effector proteins. One such effector is the Early Autosomal Autoantigen 1 (EEA1) that enhances early endosome fusion. Both EEA1 and Rabenosyn-5 contain an Nterminal $\mathrm{C}_{2} \mathrm{H}_{2}$ zinc finger, both of which bind to Rab5. In Rabenosyn-5 this is in addition to the two helical hairpins already discussed above, so that this protein has three separate Rab-binding sites. In EEA1 the Zn finger is followed by a coiled-coil and a FYVE domain that interacts with inositol phospholipids and also contributes to a second, weak Rab-binding site (Lawe et al. 2000).

The EEA1 Zn finger forms a $\beta \beta \alpha$ fold, comprising a short $\beta$-hairpin and a single $\alpha$ helix cross-braced by a $\mathrm{Zn}^{2+}$ ion (Mishra et al. 2010). All of these secondary structural elements interact with the Rab5 protein (Figure 4.5), contacting both switch regions and the interswitch via a number of hydrophobic interactions and a few polar contacts. In switch 1 , Thr52 $2^{\text {Rab }}$ contacts Glu39 ${ }^{\text {EEA1 }}$, Ile $53^{\text {Rab }}$ contacts Phe $41^{\text {EEA1, }}$ Ala56 $6^{\mathrm{Rab}}$ contacts Glu39 ${ }^{\mathrm{EEA} 1}$ and Phe $57^{\mathrm{Rab}}$ contacts the mainchain of Ser $38^{\mathrm{EEA} 1}$.

URL: http:/mc.manuscriptcentral.com/33̈mg Email: pfeffer@biochem.wisc.edu 
$\operatorname{Trp} 74^{\mathrm{Rab}}$ in the interswitch region makes hydrophobic contacts with Pro44/Met47/Ile$42^{\mathrm{EEA} 1}$. In switch 2 Tyr82 ${ }^{\mathrm{Rab}}$ packs alongside Phe $41^{\mathrm{EEA} 1}$, Leu84 ${ }^{\mathrm{Rab}}$ contacts Phe41/Leu56/Phe $57^{\text {EEA1 }}$ and Met8 $8^{\text {Rab }}$ packs against Leu56/Tyr60 ${ }^{\text {EEA1 }}$. The third hydrophobic triad residue, Tyr89 ${ }^{\mathrm{Rab}}$ contacts Pro $44^{\mathrm{EEA} 1}$ and forms a hydrogen bond with the mainchain of Ile $42^{\mathrm{EEA} 1}$. The final interaction is a hydrogen bond formed between $\operatorname{Arg} 91^{\text {Rab }}$ and Tyr60 ${ }^{\text {EEA1 }}$.

The Rab5 group includes Rab5, Rab21 and Rab22 and the Rab5 residues involved in the EEA1 contact surface are conserved within this group. Further, the EEA1 residues involved are conserved between EEA1 and Rabenosyn-5 Zn fingers, suggesting that the latter will bind similarly. Surface plasmon resonance (SPR) experiments showed that the EEA1 and Rabenosyn-5 Zn fingers bind to Rab5 with $2.4 \mu \mathrm{M}$ and $4.8 \mu \mathrm{M} K_{\mathrm{d}}$ respectively, and to Rab22 with $14 \mu \mathrm{M}$ and $63 \mu \mathrm{M} \mathrm{Kd}$. There was no detectable binding to any other Rabs (Mishra et al. 2010). Mutations that reduced the affinity of Rab5 for EEA1 included A56E, which is predicted to cause a steric clash with EEA1 Glu39 and M88A/M88S, which disrupt the hydrophobic pocket that buries the Met sidechain. These substitutions were designed to change the Rab5 into a Rab that contains Rab4/Rab11-like sequences. As the other Rab5 family member Rab21 does not bind, the only residue that contacts that is not conserved in Rab21 was mutated in the G54Q mutant and this impairs the binding, presumably due to the substitution of a larger group in the interface.

As Rab4 does not bind to the EEA1 and Rabenosyn-5 Zn fingers an attempt was made to switch the specificity of Rab4 by site-directed mutagenesis. All of the residues in the switch regions that contact EEA1 were mutated to their Rab5 counterpart but this was not sufficient to achieve high affinity binding (Mishra et al. 2010). In addition, residues in Rab4 $\alpha 1, \beta 1, \beta 2, \alpha 3$ and $\beta 4$ were replaced to their Rab5 counterparts. Only by replacing these core residues, which pack behind the switch regions and may help to stabilize the switch conformations, was a Rab4 variant generated that bound to EEA1 with an affinity comparable to that of Rab5. This work demonstrates that the plasticity of the switches can be a driver for specificity, since they can adopt different conformations even when they have the same sequence if the residues behind them are not the same. 


\subsubsection{Rab8-OCRL}

OCRL is the protein whose defect is responsible for Lowe syndrome, or oculocerebrorenal of Lowe. It is an inositol 5-phosphatase that dephosphorylates $\mathrm{PI}(4,5) \mathrm{P}_{2}$ and $\mathrm{PI}(3,4,5) \mathrm{P}_{3}$ at the 5-position and is located in the Golgi, early endosomes and plasma membrane ruffles. OCRL is localized to endosomes and Golgi by Rab proteins and it has been shown that Rab binding also stimulates the phosphatase activity of OCRL (see (Pirruccello \& Pietro De Camilli 2012) for a review). OCRL also binds to clathrin and presumably has a role in endocytosis. The OCRL protein contains a central catalytic domain, which is followed by an ASH domain (for ASPM-SPD-2-Hydin) and the Rab binding site includes the C-terminal helix of the phosphatase domain as well as the ASH domain.

OCRL has an unusually broad binding specificity for Rab proteins, unlike most Rab effectors, which bind to only one Rab or class of Rabs. The binding of Rab1b, Rab3a, Rab5a, Rab6a, Rab8a, Rab7, Rab13, Rab14 and Rab3 was tested using fluorescence polarization and only Rab7 failed to bind (Hou et al. 2011). Rab8a bound with the highest affinity (around $1 \mu \mathrm{M} \mathrm{Kd}$ ) while Rab1b, Rab5a and Rab6a bound with around $3 \mu \mathrm{M} \mathrm{Kd}$.

The structure of the Rab8-OCRL complex shows that the ASH domain adopts an Iglike fold, which is extended at the $\mathrm{N}$-terminus by a long $\alpha$-helix from the phosphatase domain (Figure 4.6). Both the ASH domain and the long $\alpha$-helix contact Rab in two distinct contact sites. The helix contacts residues in the interswitch region, using polar interactions to form hydrogen bonds between Ile $47^{\text {Rab }}$ mainchain and Asp559 ${ }^{\text {OCRL }}$ and Thr49 $9^{\text {Rab }}$ and Asp555 ${ }^{\text {OCRL }}$. The OCRL helix also contacts residues N-terminal to switch 1: Ser2 $9^{\mathrm{Rab}}$ mainchain hydrogen bonds to Arg552 ${ }^{\mathrm{OCRL}}$ and Glu30 $0^{\mathrm{Rab}}$ forms a salt bridge with Arg556 ${ }^{\mathrm{OCRL}}$. All of the switch contacts are formed with the OCRL ASH domain, mainly via the ASH $\beta 9$ strand. This strands does not form an extensive intermolecular $\beta$-sheet with Rab8 $\beta 2$, although there are a pair of backbone-backbone hydrogen bonds between Ile $43^{\text {Rab }}$ and Asp $666^{\text {OCRL }}$, where the two strands come together (Figure 4.6B). As most of the interactions are outside this region we have chosen to class this effector complex structure as 'other' rather than 'intermolecular 
$\beta$-sheet'. The other switch 1 contacts include Ile- $41^{\text {Rab }}$, whose mainchain forms a hydrogen bond with Arg-570 ${ }^{\mathrm{OCRL}}$ and whose sidechain is packed against Phe $668^{\mathrm{OCRL}}$, Gly42 ${ }^{\text {Rab }}$, which also contacts Phe $668^{\text {OCRL }}$ and Phe $45^{\text {Rab }}$ which contacts Gly $664^{\text {OCRL }}$. In switch 2, contacts are formed between Arg-69 ${ }^{\text {Rab }}$, which forms a salt bridge with Glu571 ${ }^{\text {OCRL }}$, Phe $70^{\text {Rab }}$, which is in the hydrophobic interface involving Phe $668^{\text {OCRL }}$ and Tyr $77^{\text {Rab }}$ which forms a hydrogen bond with As-666 ${ }^{\text {OCRL }}$.

The most striking feature of the Rab8-OCRL complex is that, unlike most Rab effectors, it does not involve $\alpha$-helices. Furthermore, of the hydrophobic triad residues only Phe45 makes hydrophobic contacts: Trp62 does not contact OCRL at all and Tyr77 only contacts via a hydrogen bond. It is thought that effectors are specific for particular Rab family members based on the conformation of the hydrophobic triad residues, which show differences between the Rab families, even when the triad sequences are conserved (reviewed in (Khan \& Ménétrey 2013)).

\subsection{Ran and its Effectors}

The Ran family is unusual among the small $G$ protein families in that it has only one member, the Ran protein itself. It also has the property that its GTPase cycle is compartmentalized, since its GEF and GAP are separated by the nuclear membrane. The exchange factor is in the nucleus, and the Ran-GTP formed there binds to its effector proteins: exportin binds along with export cargo while importin releases its import cargo and binds alone. The Ran-effector complexes then pass through the nuclear pore into the cytoplasm, where the RanBP1 or related proteins compete with effector binding, dissociating the complexes. This leaves the export cargo free to dissociate from exportin and diffuse into the cytoplasm, while importin can pick up a cargo destined for the nucleus, binding either directly or indirectly via adaptor proteins, and escort it though the nuclear pore. The RanBP1-Ran complex can then interact with RanGAP, whereupon the GTP is hydrolysed and free Ran GDP can reenter the nucleus for the cycle to begin again (see (Cook et al. 2007) and (Güttler \& Görlich 2011) for reviews). 
The structures of Ran in complex with both importins and exportins have been solved as well as the complex with RanBP2. RanBP2 is a PH domain effector and will be discussed with other $\mathrm{G}$ protein-PH domain interactions in section 5.6. The importin and exportin proteins are related in sequence and belong to the $\beta$-karyopherin family, interacting with Ran in a broadly similar way (Figure 4.7). The karyopherins are composed of around 20 HEAT repeats, which are formed by 2 antiparallel $\alpha$-helices ( $\alpha \mathrm{A}$ and $\alpha \mathrm{B}$ ) that stack together to form a right-handed solenoid. Between each HEAT repeat the helical hairpins are linked by a loop or by a third helix. The twisted crescent-shaped kayopherin is formed with the HEAT repeat helices running more or less parallel, with $\alpha \mathrm{B}$ on the concave surface and $\alpha \mathrm{A}$ on the convex surface of the crescent. The adjacent HEAT repeats are rotated by about $15^{\circ}$ with respect to each other but there are two clusters of larger angles so the protein forms two arches, known as the N-terminal arch and the C-terminal arch. Ran binds in the centre of the crescent and thus makes contacts with both arches.

There are several Ran-importin structures solved and here we will describe the structure that includes a full-length importin, Kap95p (Lee et al. 2005), since it includes an interface that is not present in structures solved using shorter importin constructs (Vetter et al. 1999b; Chook \& Blobel 1999). Ran and importins make three distinct interfaces, two within the $\mathrm{N}$-terminal arch and one in the $\mathrm{C}$-terminal arch (Figure 4.7B). The importin protein will be denoted as H1-H18 to identify the HEAT repeat involved in the interaction. The $\mathrm{C}$-terminal arch makes interactions with residues in the N-terminus and switch 1 of Ran: $\operatorname{Arg} 29^{\text {Ran }}$ forms bonds with Gln570/Gln567 ${ }^{\mathrm{H} 13}$, Phe $35^{\mathrm{Ran}}$ contacts Phe613/Ala612 ${ }^{\mathrm{H} 14}$ and Lys $37^{\mathrm{Ran}}$ forms hydrogen bonds/salt bridges with Glu615/Asp616 ${ }^{\mathrm{H} 14}$ and $\mathrm{Gln} 650^{\mathrm{H} 15}$. There are interactions between residues in helix $\alpha 5$ of Ran and the last portion of the loop that immediately precedes this helix: Lys152, Asn154, Tyr155, Asn156, Tyr159 and Tr163 are all involved in hydrogen bonds or salt bridges to HEAT domains H12-H14. Switch 2 makes contacts with HEAT repeats at the start of the N-terminal arch: Glu $70^{\text {Ran }}$ forms a salt bridge with Lys $76^{\mathrm{H} 2}$, Leu $75^{\text {Ran }}$ contacts Ile14/Glu26 ${ }^{\mathrm{H} 1}$, As- $77^{\text {Ran }}$ forms hydrogen bonds with Lys66/Asn6 $7^{\mathrm{H} 2}$, Tyr79 ${ }^{\mathrm{Ran}}$ hydrogen bonds with Glu26 ${ }^{\mathrm{H} 1}$, Ile $81^{\text {Ran }}$ packs against Ile $14^{\mathrm{H} 1} / \mathrm{Ile} 59^{\mathrm{H} 2}$ and Gln $82^{\text {Ran }}$ forms hydrogen bonds with Glu $56^{\mathrm{H} 2} / \operatorname{Arg} 110^{\mathrm{H} 3}$. There are also polar contacts formed between $\operatorname{Arg} 106$ and Arg110 
at the end of helix $\alpha 3$ in Ran and HEAT H4. The third contact formed between Ran and the importin does not involve the switches at all and so is not nucleotide dependent but instead serves to increase the affinity of the complex. In this contact a basic patch on Ran around helix $\alpha 4$, comprising residues Lys134, His 139, Arg140 and Lys 141 contacts an acidic loop that connect the two helices in H8. The details of the interactions between the different importins and Ran are all slightly different but the general contact areas remain the same.

The large interaction interface between Ran and the importins means that the affinity of the interaction is very high, 230 pM for Kap95p (Hahn \& Schlenstedt 2011). This high affinity allows Ran-GTP to compete effectively with import cargo interactions with the importins in the nucleus, so that cargo is released. The high affinity has a drawback, it means that binding to RanBP1 in the cytoplasm is necessary to dissociate the Ran-importin complex before RanGAP is able to bind and stimulate GTP hydrolysis.

There are several Ran-exportin complexes whose structures have been solved in ternary complexes with various cargos. The first of these was of an importin adaptor protein, Kap60p (the cargo) in a ternary complex with the Cse1p exportin (or CAS) and Ran·GTP (Matsuura \& Stewart 2004). This structure showed that the exportin has a superficially similar structure to the importin structures, as was expected, but that the details of its interactions with Ran differ. (Figure 4.7 C and D) There are only two interaction areas in the Ran-exportin interface, one of these is broadly similar to the contact between switch 2 and the N-terminal arch in the importin complex and involves mostly polar contacts between Arg $76^{\text {Ran }}$, Asp $77^{\text {Ran }}$, Tyr79 Ran , Ile81 Ran, Gln $82^{\text {Ran }}$ and Arg1 10 Ran and the HEAT1-3 in the exportin. There are also hydrophobic contacts in this interface involving Leu $75^{\text {Ran }}$ and Ile $81^{\text {Ran }}$. The second contact site has no equivalent in the importin complex and involves HEAT13-14 and a long loop within HEAT19. HEAT13-14 forms salt bridges with Lys37 ${ }^{\text {Ran }}$ in switch 1 and Lys132 ${ }^{\text {Ran }}$. The HEAT19 loop contacts switch 1 via Tyr39 ${ }^{\text {Ran }}$ but also pokes into the nucleotide binding site and inserts a Phe sidechain to stack against the guanine base. This would presumably inhibit nucleotide dissociation. The HEAT19 loop is also pinned into position by polar contacts with Lys $123^{\text {Ran }}$ and Asp $128^{\text {Ran }}$. 
In the Ran-importin complex the third contact region involved the basic patch of Ran that interacted with an acidic region in importin, forming a number of salt bridge and hydrogen bonds. The Ran basic patch is involved in directly contacting the cargo in the exportin ternary complex with Kap60p. This involves interactions between Arg95 ${ }^{\text {Ran }}$, Lys99 ${ }^{\text {Ran }}$, Lys $130^{\text {Ran }}$, Lys $132^{\text {Ran }}$ and Lys $134^{\text {Ran }}$ and polar residues in the cargo so that a network of hydrogen bonds and salt bridges are formed.

Other structures of Ran-exportin-cargo complexes have shown that the same region of Ran can be involved in other cargo interactions. For example, Lys $132^{\text {Ran }}$ and Lys $134^{\text {Ran }}$ form salt bridges with pre-microRNA in the exportin complex (Okada et al. 2009). In a tRNA exportin complex Arg97 ${ }^{\text {Ran }}$, Lys $101^{\text {Ran }}$ and Lys $134^{\text {Ran }}$ all form salt bridges with the RNA moiety (Cook et al. 2009). Ran does not always contact the cargo however, for example in the Ran-Importin13-eIF1A complex the two species are close but not actually touching (GrUnwald et al. 2013). The free state of the Importin13 was also solved and was found to be more open than the complex state, so that although Ran does not bind to the cargo directly it is necessary to stabilize the closed conformation of the exportin (GrUnwald et al. 2013). This mechanism was also proposed when the Ran-CRM1-Snurportin1 complex was solved and Ran was shown to form no contacts with the Snurportin1 cargo (Monecke et al. 2009). The detailed mechanism of exportin activation by Ran is not universal however, since structural work on the cytoplasmic form of Csel shows that it is clamped closed in the free form and that Ran is required to open before cargo can bind (Cook et al. 2005).

The contact regions between Ran and exportins also vary between the different complexes. In the Ran-Xpot-rRNA complex, the N-terminal arch of Xpot exportin interacts with switch 2 of Ran, while a large loop in HEAT9 contacts the basic patch on Ran (Cook et al. 2009). The C-terminal arch of Xpot interacts with switch 1 using the loops within HEAT13 and HEAT17. Although the Ran regions involved in the interactions are broadly similar to those in Cse1 complex, the basic patch is involved in contacting Xpot. The Exp5-Ran-miRNA complex also shows a different means of Ran interaction: the C-terminal arch no longer interacts with switch 1 and instead the N-terminal arch interacts with both switches (Okada et al. 2009). Perhaps unsurprisingly, the most divergent interactions are those of Ran with CRM1, where 
Ran does not contact the cargo (Monecke et al. 2009). Instead, Ran is engulfed by the exportin, which makes extensive contacts with the $\mathrm{G}$ protein using 4 regions of CRM1. HEATS1-5 contact switch 2, Ran helix $\alpha 3$ and part of the basic patch, while HEATS7-9 also contact the basic patch and strand $\beta 6$. HEAT9 contains a long $\beta$ hairpin insertion that locks Ran against the $\mathrm{N}$ - and C-terminal HEAT repeats, binding to switch 1 and the loops involved in the guanine binding. The fourth region of CRM1 that binds to Ran involves HEAT17 and HEAT19, which bind to both switch regions.

\section{Effectors that interact via a PH domain}

The pleckstrin homology $(\mathrm{PH})$ domain fold includes a 7-stranded, anti-parallel $\beta$ sheet that is strongly bent so that it forms an orthogonal $\beta$-sandwich, followed by a Cterminal $\alpha$-helix that blocks one end of the sheet (see (Scheffzek \& Welti 2012) for a review). Although originally thought of as a phospholipid-binding module, particularly for phosphorylated inositols, it is now clear that $\mathrm{PH}$ domains are also involved in several protein-protein interactions. There are several examples of effectors that utilize a $\mathrm{PH}$ domain to bind to small $\mathrm{G}$ protein and the most intriguing feature of the structures is their variety (Figure 5.1). In these cases, two folds are brought together, a G domain and a PH domain, and it might be predicted, particularly from an evolutionary perspective, that they would interact in a similar manner. It is clear that this is not the case and that the interactions did not originate from a common ancestor. The diversity of these structures illustrates the features of both of these domains that make them unique. PH domains, despite being rather small (around 100 amino acids) do not use the same interaction surface to contact interacting partners. This is very clear when the structures are posed so that the $\mathrm{PH}$ fold is in the same orientation in each (Figure 5.2). Small G proteins, on the other hand, use a similar interface to contact most effector proteins but use the plasticity of that interface, which encompasses the switch regions, to allow them to make contacts with any secondary structural element in the binding partners. In the case of the PH domains we can see that in one case there is an intermolecular $\beta$-sheet formed (RalAExo84, see section 2.1), that there are helix-helix interactions between the $\mathrm{PH}$ domain and switch 2 (e.g. Arf1-ARHGAP21, Rac2-PLC $\gamma 2$ ), that there are loops interacting 
with the switches or that the sides of the PH domain $\beta$-sheets interact with the switches.

There are examples of $\mathrm{PH}$ domains that bind $\mathrm{G}$ proteins from four of the five subfamilies, the exception being the Rab subfamily. The Ras subfamily example, Exo84, also forms an intermolecular $\beta$-sheet and has been discussed previously (see Section 2.1.11.

\subsection{Rac1-phospholipase C- $\beta 2$}

PLC $\beta 2$ is one of a family of 13 enzymes that catalyse the hydrolysis of $\mathrm{PI}(4,5) \mathrm{P}_{2}$ into $\mathrm{DAG}$ and $\mathrm{IP}_{3}$, leading to activation of protein kinase $\mathrm{C}$ and release of intracellular $\mathrm{Ca}^{2+}$ stores. The PLC family proteins generally contain a conserved core that includes a PH domain, EF hands, the catalytic domain and a C2 domain. Rac proteins can directly activate PLC $\beta 2$ and Rac1-3 bind to PLC $\beta 2$ with 5-10 $\mu \mathrm{M} K_{\mathrm{d}}$ (Snyder et al. 2003).

The structure of the entire core of PLC $\beta 2$ in complex with Rac1 has been solved (Jezyk et al. 2006). The PH domain of PLC $\beta 2$ is the only domain in the core involved in contacting Rac 1 and it sits at the center of a triangle whose edges comprise the other domains in the core and contacts all of them as well as the small $\mathrm{G}$ protein. The contacts with the $\mathrm{PH}$ domain only involve the switch regions and the interswitch of Rac1 (Figure 5.2B). There are a number of hydrophobic interactions at the interface that are supported by hydrogen bonds and salt bridges at the periphery. In switch 1 , Val36/Phe3 $7^{\mathrm{Rac}}$ are involved in packing against Arg22/Gln52/Tyr118 ${ }^{\mathrm{PLC}}$ and Asn $39^{\text {Rac }}$ hydrogen bonds with Gln52 ${ }^{\text {PLC }}$. In the interswitch region, Ser4 ${ }^{\text {Rac }}$ hydrogen bonds to Lys $54^{\mathrm{PLC}}$ and Trp56 $6^{\mathrm{Rac}}$ is also packed against Gln52/Tyr118 ${ }^{\mathrm{PLC}}$. In switch 2 , Tyr64 ${ }^{\text {Rac }}$ also contacts Tyr $118^{\text {PLC }}$ in the hydrophobic interface. Arg66 ${ }^{\text {Rac }}$ forms polar contacts with Asn86 ${ }^{\mathrm{PLC}}$, while Leu67/Leu70 ${ }^{\mathrm{Rac}}$ pack against Ile24/Val84 ${ }^{\mathrm{PLC}}$ and Ser71 ${ }^{\text {Rac }}$ hydrogen bonds to Gln52 ${ }^{\text {PLC }}$.

Most of the contacting residues on Rac1 are conserved in RhoA and Cdc42, neither of which bind and activate PLC $\beta 2$. Two differences are Ser41, which is Ala in Cdc42 and Val in RhoA, and Trp56, which is conserved in RhoA and a Phe in Cdc42. The 
combination of these two changes, which involve loss of a hydrogen bond and destabilization of the hydrophobic packing, must be sufficient to reduce the affinity for Cdc42. In the case of RhoA, the only change would be the loss of the hydrogen bond involving Ser41. It was however observed that while Cdc42 and Rac1 both have similar surface electrostatic potentials, RhoA is significantly negative and that this could repel a negative lobe in the PH domain of PLC $\beta 2$, preventing complex formation (Jezyk et al. 2006).

The effects of mutations of Rac 1 residues in the interface was assessed in COS- 7 cells using inositol phosphate accumulation as a PLC $\beta 2$ readout (Jezyk et al. 2006). Mutants F37A, W56A, L67A and L70A all reduced the PLC $\beta 2$ activation by Rac1. S41A was not tested, so the contribution of its hydrogen bond to the complex stability cannot be assessed.

\subsection{Rac2-phospholipase $\mathrm{C}-\boldsymbol{\gamma} 2$}

PLC $\gamma 2$ is also activated by Rac proteins and although it also has a PH domain at the $\mathrm{N}$-terminus, this does not appear to be the crucial domain for binding to the small $\mathrm{G}$ protein. Rather, PLC $\gamma 2$ contains a second PH domain in the center of its sequence, which has an unusual topology in that it is split by two SH2 domains and one SH3 domain (Walliser et al. 2008). This split PH domain is responsible for the activation of PLC $\gamma 2$ and, when expressed with a flexible linker instead of the 3 domains that split the $\mathrm{PH}$, the resulting 'spPH' can bind to Rac2 with $13 \mu \mathrm{M}$ affinity, comparable to the affinity of longer PLC $\gamma 2$ constructs.

The structure of the spPH-Rac2 complex shows that the orientation of the $\mathrm{PH}$ domain with respect to the small $\mathrm{G}$ protein is very different from the PLC $\beta 2$-Rac1 orientation (Bunney et al. 2009) (see Figure 5.1B and C and Figure 5.2). The interface on Rac2 that interacts with the $\mathrm{PH}$ domain is however almost the same as that in Rac1 that contacts PLC $\gamma 2$. In switch 1, Val36/Phe37 ${ }^{\text {Rac }}$ make hydrophobic contacts with Lys862/Val893/Phe897 ${ }^{\text {PLC }}$, Asp38 ${ }^{\text {Rac }}$ forms a salt bridge with Lys862 ${ }^{\text {PLC }}$ and Asn $39^{\text {Rac }}$ forms a hydrogen bond with Gln $901^{\mathrm{PLC}}$. In the interswitch region Ser4 $1^{\text {Rac }}$ is again involved but this time contacts Trp908 ${ }^{\mathrm{PLC}}$, while Trp56 ${ }^{\mathrm{Rac}}$ contacts Gln901/Arg904 ${ }^{\mathrm{PLC}}$. The hydrophobic switch 2 residues are also in the hydrophobic 
interface formed by switch 1 and the PH domain: Tyr64/Leu67/Leu70 Rac are packed against Val893/Phe-97 ${ }^{\text {PLC }}$. Ser71 ${ }^{\text {Rac }}$ does not form a hydrogen bond in this complex, although it also contacts Phe $897^{\text {PLC }}$.

A number of mutants in Rac2 were also generated and their affinities for spPH were measured (Bunney et al. 2009). Their effects were also validated in an in-cell PLC $\gamma 2$ activation assay. Mutations that showed reduced or no binding were: V36A, F37A, D38A, Y40C, W56A, Y64A, L67A and L70A. The effect of V36A was smaller than the other mutations but it reduced the affinity around 5-fold. These residues are all in the interface, with the exception of Y40C, which is likely to disrupt the structure of switch 1 .

The D38A and Y64A mutations of Rac1 were also tested for their ability to activate PLC $\beta 2$ via its N-terminal PH domain (Jezyk et al. 2006) and had little effect. Thus even though the regions of Rac1 and Rac2 that contact these two different $\mathrm{PH}$ domains are similar, the contribution of the residues to binding is not the same. This implies that mutations in Rac1 or Rac 2 could be designed that discriminate between the two PH domains and hence their associated PLC activation. A careful comparison of the structures of Rac1-PLC $\beta 2$ and Rac2-PLC $\gamma 2$ suggested that Leu67 to a negative residue could be accommodated in the PLC $\beta 2$ complex better than in the PLC $\gamma 2$ complex (Bunney et al. 2009). The L67E mutant showed excellent discrimination in PLC activation assays in vitro and in cells.

An attempt was made to turn Cdc42 into a PLC $\gamma 2$ binding protein by mutating Phe56 into Trp, which is involved in hydrophobic contacts in the Rac2-PLC $\gamma 2$ interface. The Rac2 W56F mutation reduced the affinity around 10-fold (Bunney et al. 2009). The Cdc42 F56W mutation binds with a $40 \mu \mathrm{M}$ Kd (compared to almost $300 \mu \mathrm{M}$ for wildtype Cdc42). In activation assays however, the Cdc42 F56W mutant was unable to stimulate the PLC $\gamma 2$ catalytic activity. This implies that other regions of Rac are necessary for full catalytic activation. It is possible that this also involves other regions of the PLC $\gamma 2$ protein, since longer PLC $\gamma 2$ constructs bind around 4-fold more tightly than the spPH construct (Walliser et al. 2008). 


\subsection{Rho1p-Sec3}

The last PH domain interaction with a Rho family protein comes from the yeast exocyst complex component Sec3, in complex with Rholp. The yeast exocyst complex, like its mammalian counterpart, is octameric and various components interact with small $\mathrm{G}$ proteins but the details of the intra-octamer interactions are different (reviewed in (Heider \& Munson 2012). In mammalian cells, RalA and Rab11 on vesicles interact with exocyst components Exo84/Sec5 and Sec15 respectively, while Exo70 interacts with TC10 at the plasma membrane, as well as binding to $\mathrm{PI}(4,5) \mathrm{P}_{2}$ so that together the exocyst octomer tethers exocytic vesicles to the target membrane. In yeast there are no Ral protein orthologues and interactions with vesicles are mediated solely between the Rabs, Sec4, and Sec15. At the plasma membrane Exo70 contacts Rho3p, while Sec3 interacts with Rho1 or Cdc42. Both Exo70 and Sec3 also bind to PI(4,5) $\mathrm{P}_{2}$ (see (Yamashita et al. 2010) Supp Fig 1 for a comparative sketch). The exocyst complex is involved in budding in yeast, where a number of components are transported to the bud tip by polarized exocytosis.

The N-terminus of Sec3 interacts with Rho1/Cdc42 and when the structure was solved it was clear that it forms a PH domain fold, despite having no sequence homology that allowed this to be predicted (Yamashita et al. 2010). There are three extra $\alpha$-helices and an extra $\beta$-strand in the structure, but only the longer, $\mathrm{N}$-terminal helix (residues 76-94) contacts Rholp (Figure 5.1D). Both Rhol switch regions are involved in the contact site. In switch 1 , Pro $41^{\text {Rho }}$ is packed against Phe $77^{\mathrm{Sec}}$, Val43 ${ }^{\mathrm{Rho}}$ contacts Asn $201^{\mathrm{Sec}}$, Phe $44^{\mathrm{Rho}}$ is involved in hydrophobic contacts with Leu131/Lys $136^{\mathrm{Sec}}$ and Glu45 $5^{\mathrm{Rho}}$ forms a salt bridge with Lys $136^{\mathrm{Sec}}$. In the interswitch region the only contact is with $\operatorname{Trp} 63^{\mathrm{Rho}}$, which is involved in contacts with Leu131/Glu132 ${ }^{\mathrm{Sec}}$. In switch 2 , Tyr $71^{\text {Rho }}$ is involved in hydrophobic contacts with Phe77/Leu $78^{\text {Sec }}$ and Arg73 ${ }^{\text {Rho }}$ forms a salt bridge with Glu199 ${ }^{\text {Sec }}$. GST pulldown assays were used to show that mutations in several Rholp residues can disrupt the binding: V43A, F44A, Y71A and R73A did not pull down Sec3.

As Sec3 is also known to bind inositol phospholipids, the discovery of a $\mathrm{PH}$ domain fold at its N-terminus immediately suggested a means by which PIP $_{2}$ could bind. Sure enough, an analysis of the surface potential of the Sec3 PH domain showed that there 
is a cluster of basic residues and therefore positive charge at the open end of the $\mathrm{PH}$ domain $\beta$-sheet. This region is similar to that used for phospholipid binding in other $\mathrm{PH}$ domains and mutation of these basic residues reduced phospholipid binding in overlay assays (Yamashita et al. 2010).

\subsection{Arf6-Grp1}

Grp1 is a member of the cytohesin family of Arf exchange factors, which also includes Arno and cytohesin-1. These proteins contain a Sec7 domain, which has exchange activity for Arf1 and Arf6, followed by a PH domain that binds to inositol phospholipids and an overlapping $\mathrm{C}$-terminal helix $(\mathrm{CtH})$ and polybasic region (PBR) (reviewed in (Stalder \& Antonny 2013). The cytohesins are autoinhibited by the Sec7$\mathrm{PH}$ linker and by the $\mathrm{CtH}$ and PBR, and binding of Arf6 and $\mathrm{PIP}_{3}$ relieves the inhibition and allows stimulation of exchange.

The Arf6 binding region was delineated using SPR and the minimal binding fragment includes just 5 residues from the Sec7-PH linker and all of the CtH/PBR (Malaby et al. 2013). This fragment binds with an affinity around $10 \mu \mathrm{M}$ to both full-length and N-terminally truncated Arf6.

The complex was crystallized in the presence of $\mathrm{IP}_{4}$ and the structure shows that all three moieties in the Grp1 contact Arf6 i.e. the short section of linker (255-264), the PH domain and the CtH/PBR (381-397) (Malaby et al. 2013). The center of the interface is formed by the $\mathrm{PH}$ domain, strands $\beta 1-\beta 4$ and $\beta \mathrm{i} 1$ and $\beta \mathrm{i} 2$, which are a $\beta$ hairpin insertion found in the cytohesins (Figure 5.1E). The PH domain mainly contacts the switch regions of Arf6: Phe $47^{\text {Arf }}$ contacts Pro309/Cys342 ${ }^{\text {Grp }}$, Asn $48^{\text {Arf }}$ hydrogen bonds to Lys $340^{\mathrm{Grp}}$ and Val49 ${ }^{\mathrm{Arf}}$ contacts Glu352 ${ }^{\mathrm{Grp}}$. In the interswitch the conserved hydrophobic Trp62 ${ }^{\text {Arf }}$ contacts Cys342/Val350 Grp . In switch 2 Asp69 Arf forms a salt bridge with Asp290 ${ }^{\text {Grp }}$ and Leu $73^{\text {Arf }}$ is packed against Tyr294 ${ }^{\text {Grp }}$. This is followed by several interactions: His $76^{\text {Arf }}$ contacts Pro304/Ile307/Val350 ${ }^{\mathrm{Grp}}$ and hydrogen bonds to Thr394 ${ }^{\mathrm{Grp}}$; Tyr $77^{\text {Arf }}$ contacts Ile $307^{\mathrm{Grp}}$ and Thr79 $9^{\mathrm{Arf}}$ hydrogen bonds to the mainchain of Gly $348^{\mathrm{Grp}}$. The N-terminal linker section of the Grp1 fragment makes contacts with two residues just C-terminal to switch 2, the hydrogen bond with His76 listed above and contacts between $\operatorname{Arg} 75^{\text {Arf }}$ and Leu258 Grp and the 
mainchain of Asp257 $7^{\mathrm{Grp}}$. The $\mathrm{CtH} / \mathrm{PBR}$ makes more extensive contacts, mainly to residues in switch 1 and N-terminal to the switch: Tyr31, Leu35, Gln37, Val39 and Ile42 all contact residues in the $\mathrm{CtH}$, which runs more or less parallel to $\alpha 1$ of the Arf6 G domain. Despite the basic nature of the $\mathrm{CtH} / \mathrm{PBR}$, there is only a single acidic residue in Arf6 that makes a salt bridge: Glu-50 in the interswitch with Lys392 ${ }^{\text {Grp }}$. Most of the contacts in this region are hydrophobic or weak hydrogen bonds.

A comparison of the structure of Arf6-Grp1 with the autoinhibited structure of Grp1 (DiNitto et al. 2007) showed that Arf6 binding to the N-terminal linker and the $\mathrm{CtH} / \mathrm{PBR}$ caused a rotation in these regions away from the $\mathrm{Sec} 7$ active site, thus demonstrating how Arf6 can allosterically activate the exchange factor. The $\mathrm{IP}_{4}$ binding site on the PH domain is on the opposite face to the Arf6 binding site and it is not immediately obvious from the structure why $\mathrm{IP}_{4}$ is necessary for high affinity Arf6 binding. It was proposed that $\mathrm{IP}_{4}$ binding might alter the conformation of the $\beta \mathrm{i} 1$ and $\beta \mathrm{i} 2$ hairpin insertion, allowing formation of a structure that is more competent to bind to Arf6 (Malaby et al. 2013).

\subsection{Arf1-ARHGAP21}

ARHGAP21 is an Arf1 and Arf6 effector that is also a GAP for the Rho family. As Arf1 is at the Golgi, its recruitment of ARHGAP21 allows actin rearrangements to occur to facilitate Golgi organization and vesicle budding. ARHGAP21 acts on Cdc42, which controls actin via the Arp2/3 complex and thus provides a link between Arf and Cdc42 signaling (Dubois et al. 2005).

ARHGAP21 contacts Arfs using a PH domain with a C-terminal extension, which forms a long $\alpha$-helix of around 20 amino acids (residues 1041-1063) (Ménétrey et al. 2007). Both the PH domain and the C-terminal helix contact the switch regions of Arf1 (Figure 5.1F): the PH domain forms most of the switch 1 contacts, using the fifth $\beta$-strand and the following loop and part of the PH domain $\alpha$-helix, while the $\mathrm{C}$ terminal helix contacts the interswitch and switch 2 . Affinity measurements using analytical ultracentrifugation showed that the $\mathrm{PH}+$ extension bound tightly $\left(K_{\mathrm{d}} 55\right.$ $\mathrm{nM}$ ) but that removal of the extension reduced the affinity 650 -fold. The C-terminal helix extension lies in a groove between the two switch regions and is more or less 
parallel to the $\beta 2-\beta 3$ strands in the interswitch region. N-terminal to switch 1 in the Arf1 $\alpha 1$ helix, Tyr35 $5^{\text {Arf }}$ contacts Tyr999 ${ }^{\text {GAP21 }}$, while in switch 1 Val43/Thr45 ${ }^{\text {Arf }}$ also contact Tyr999 ${ }^{\mathrm{GAP} 21}$. The other switch 1 contacts with the PH domain are: Thr44 ${ }^{\text {Arf }}$ with $\operatorname{Arg} 1024^{\text {GAP21 }}$ and Ile $46^{\text {Arf }}$ with Ile $1031^{\text {GAP21 }}$, which are contacts involving the $\alpha$ helix of the PH domain and Glu54 ${ }^{\text {Arf }}$, which forms a hydrogen bond with Ser $1000^{\mathrm{GAP} 21}$. The remainder of the contacts involve the C-terminal $\alpha$-helical extension to the PH domain: in switch 1 Phe51 ${ }^{\text {Arf }}$ to $\operatorname{Arg} 1056 / \mathrm{Ile} 1057^{\mathrm{GAP} 21}$ and Val53 ${ }^{\text {Arf }}$ to Tyr1060 ${ }^{\text {GAP21 }}$; in the interswitch Trp66 ${ }^{\text {Arf }}$ to Tyr1060/Asn1061 ${ }^{\text {GAP21 }}$ and in switch $2{\text { Lys } 73^{\text {Arf }} \text { to Glu1042 }}^{\text {GAP21 }}$, Leu $77^{\text {Arf }}$ to Ile $1053^{\text {GAP21 }}$ and Tyr81 ${ }^{\text {Arf }}$ to Ile $1057^{\mathrm{GAP} 21}$.

An analysis of the sequences shows that most of the residues involved in the interaction are conserved between Arf1 and Arf6, except for Ile49, which is a Val in Arf6. The Arl family members do not have all the interface residues conserved and therefore are unlikely to bind to ARHGAP21.

A structure of the free PH domain of ARHGAP21 has been solved (PDB 2DHJ) and there are some structural rearrangements in the loop following the $\mathrm{PH} \beta$-strand 5 that contacts Arf1, centered on Tyr999. This implies that there may be some allosteric effects when Arf1 binds to ARHGAP21. GTPase assays performed using Cdc42 and longer constructs of ARHGAP21 showed that the Arf1 binding had no direct effect on the GAP activity of ARHGAP21 (Ménétrey et al. 2007). It is likely that in vivo Arf1 localized ARHGAP21 to the Golgi membrane, where it can act on membrane-bound Cdc42.

\subsection{Ran-RanBP2}

The final effector protein that uses a $\mathrm{PH}$ domain to bind to a small $\mathrm{G}$ protein is the Ran binding domain 1 (RanBD1), which is found in the related proteins RanBP1 and RanBP2. RanBP1 is a soluble Ran effector and contains a single RanBD1, while RanBP2 is a component of the nuclear pore complex and contains four RanBD1s (reviewed in (Fried \& Kutay 2003)). 
The structure of the RanBD1 from RanBP2 with Ran showed that RanBD1 is a PH domain but that it is extended at the N-terminus (Vetter et al. 1999c) and this extension also contacts Ran. Furthermore, the Ran $\mathrm{G}$ domain is extended at the $\mathrm{C}$ terminus and this extension is also involved in the interaction, so that the two proteins are involved in a 'molecular embrace' (Figure 5.2G). The large buried surface area in this complex is consistent with the high affinity of the interaction (around $5 \mathrm{nM} K_{\mathrm{d}}$ ). The Ran interaction surface involves switch 1 and the interswitch region but does not involve any interactions with switch 2 . Instead, the C-terminal extension of Ran is a third 'switch', in that the helix, which is followed by an acidic patch, contacts the G domain of Ran in the GDP-bound form. In the GTP-bound form the helix is released and can contact the RanBD1. Residues within switch 1 of Ran bind to the main part of the RanBD1 and are dominated by polar contacts: $\operatorname{Arg} 29^{\text {Ran }}$ forms a salt bridge with Glu59 $9^{\text {RanBD1}}$, Thr32 $2^{\text {Ran }}$ contacts Arg49 ${ }^{\text {RanBD1 }}$, Glu34 ${ }^{\text {Ran }}$ and Lys $38^{\text {Ran }}$ form salt bridges to Lys $58^{\text {RanBD1 }}$ and Glu56 ${ }^{\text {RanBD1 }}$ respectively. Within the interswitch region there are some backbone contacts with the RanBD1 N-terminal extension, for example between Asn5 $5^{\text {Ran }}$ and Ile2 $8^{\text {RanBD1 }}$ and between Arg56 ${ }^{\text {Ran }}$ and Pro25 $5^{\text {RanBD1 }}$. There are also a few contacts involving residues in the loop between $\beta 6$ and $\alpha 5$ of Ran: Glu158 and Trp163. The remainder of the Ran contacts involve residues beyond the G domain: the first part of $\mathrm{C}$-terminus forms an extended region that contacts the $\mathrm{PH}$ domain of RanBD1: these involve residues 169-190, which form hydrophobic interactions with the PH domain, with the exception of Gln-186 ${ }^{\text {Ran }}$, which forms a salt bridge with Lys $75^{\text {RanBD1 }}$. After this, the extra helix begins and the interactions are a mixture of hydrophobic and polar from 197-205 where the helix ends. The last few residues then contact the PH domain making hydrophobic interactions until Asp211 ${ }^{\text {Ran }}$, which forms a salt bridge with Lys $46^{\text {RanBD1 }}$. The C-terminal residues of Ran, residues 212216 , are missing in the structure but are the thought to be a crucial part of the third switch region observed in Ran. The last observed amino acid, Asp211, is the first of the acidic DEDDDL motif, which forms salt bridges in the GDP-bound form with a basic patch on Ran around 139-142. The region of RanBD1 that is close to Asp211 in the complex structure is also basic, leading to the suggestion that the final residues in the acidic motif will form salt bridges with the basic patch on RanBD1 (Vetter, et al. 1999c). The interactions with the N-terminal extension to the RanBD1 PH domain are less extensive: it contacts the very tip of the $\beta 2-\beta 3$ hairpin in Ran within the 
interswitch and lies alongside the Ran C-terminal extended region, before looping up to contact the Ran $\alpha 5$ helix.

The structure of the Ran-RanDB1 complex, when compared with structures of Ran with importins and exportins, suggests the mechanism by which RanBD1 can aid dissociation of the Ran-effector complexes and allow GAP to enter the Ran active site. The acidic C-terminal tail of Ran is not involved in contacting exportin and importin molecules and so can mediate the initial contacts with RanBD1. The RanBD1 then interacts with the basic patch on Ran as well as switch 1 and forms a tight complex, effectively competing with importins for Ran binding. The mode of exportin-cargo release may be slightly different and may vary with different cargo molecules (reviewed in (Güttler \& Görlich 2011)).

\section{Conclusions}

A comparison of all of the small $\mathrm{G}$ protein-effector complex structures suggests that specificity for binding the different effectors is driven in part by sequence difference in the $\mathrm{G}$ domain between the families. Figure 6.1 shows a heat map representing the contacts seen in the small $\mathrm{G}$ protein-effector complexes described above. Apart from the hot spots for interaction within the two switch regions, which were expected, there are common regions of interaction within the first $\beta$-strand and $\alpha$-helix ( $\beta 1$ and $\alpha 1)$ and within the interswitch region at $\beta 3$. In the Rho, Arf and Rab subfamilies there are contacts in the region around the end of $\alpha 3$ and the following loop. In the Rho family there is a region in the C-terminal helix $(\alpha 5)$ that involved in several complexes, while in the Rabs there are contacts seen in the sequences C-terminal of the $G$ domain. The Ran subfamily is clearly an outlier, making contacts more or less all the way along the sequence.

Within the subfamilies the differences are less stark and in the Rab family particularly it is clear that there is exquisite specificity for effectors even when the interacting residues are conserved. It has become clear that despite their sequence similarities the small $\mathrm{G}$ proteins have subtly different structures and dynamics and that more work is needed to understand the fine details of effector selection. Finally, it is clear that even 
amongst effectors that have some similarity in sequence or structure, the structural details of their interactions with small G proteins are difficult to predict. This is evident in the examples we have described of the intermolecular $\beta$-sheet interactions, which can be parallel or anti-parallel, the helical pairs that can be divided into six types, and the $\mathrm{PH}$ domains, which all interact in different ways.

Considering the relatively compact size of the small $\mathrm{G}$ proteins and their predominant use of one face to interact with effector proteins, it is perhaps not unexpected that they utilize all available differences to attain specificity of effector binding. The chemistry of contact sidechains is exploited: some complexes rely mainly on hydrophobic contacts; others are primarily polar, whereas still others are mixed. A huge variety of topologies exist within the effector proteins, which can be accommodated by the flexible nature of the small $\mathrm{G}$ proteins themselves and their pliant switch regions. Non-contact residues in the small $\mathrm{G}$ proteins also play a role, defining the topography of interacting surfaces. Finally the dynamics of the effector interacting surfaces on the small G proteins cannot be underplayed and almost certainly underpin some of the specificity that we cannot currently provide explanations for presently. Of course, all of the data we currently have at our disposal considers small $\mathrm{G}$ protein complexes outside their natural environment and the likely influence of membrane attachment on the vast majority of these interactions should not be underestimated.

We hope that over the next 20 years our knowledge will increase even further and perhaps we can add more structural classes to the effectors that can interact with the small $\mathrm{G}$ proteins, whose small size belies their incredible versatility.

\section{Declaration of Interest}

The authors report no declarations of interest.

\section{References}

Abdul-Manan N, Aghazadeh B, Liu GA, Majumdar A, Ouerfelli O, Siminovitch KA \& Rosen MK (1999) Structure of Cdc42 in complex with the GTPase-binding domain of the "Wiskott-Aldrich syndrome" protein. Nature 399, 379-383. 
Bae YS, Oh H, Rhee SG \& Yoo YD (2011) Regulation of reactive oxygen species generation in cell signaling. Mol Cells 32, 491-509.

Bailey LK, Campbell LJ, Evetts KA, Littlefield K, Rajendra E, Nietlispach D, Owen D \& Mott HR (2009) The structure of binder of Arl2 (BART) reveals a novel G protein binding domain: implications for function. J Biol Chem 284, 992-999.

Banerjee T, Taylor M, Jobling MG, Burress H, Yang Z, Serrano A, Holmes RK, Tatulian SA \& Teter K (2014) ADP-ribosylation factor 6 acts as an allosteric activator for the folded but not disordered cholera toxin A1 polypeptide. Molecular Microbiology 94, 898-912.

Bauer B, Mirey G, Vetter IR, García-Ranea JA, Valencia A, Wittinghofer A, Camonis JH \& Cool RH (1999) Effector recognition by the small GTP-binding proteins Ras and Ral. J Biol Chem 274, 17763-17770.

Bell CH, Aricescu AR, Jones EY \& Siebold C (2011) A Dual Binding Mode for RhoGTPases in Plexin Signalling. PLoS Biol 9.

Boriack-Sjodin PA, Margarit SM, Bar-Sagi D \& Kuriyan J (1998) The structural basis of the activation of Ras by Sos. Nature 394, 337-343.

Bosch DE, Yang B \& Siderovski DP (2012) Entamoeba histolyticaRho1 Regulates Actin Polymerization through a Divergent, Diaphanous-Related Formin. Biochemistry 51, 8791-8801.

Bunney TD, Harris R, Gandarillas NL, Josephs MB, Roe SM, Sorli SC, Paterson HF, Rodrigues-Lima F, Esposito D, Ponting CP, Gierschik P, Pearl LH, Driscoll PC \& Katan M (2006) Structural and mechanistic insights into ras association domains of phospholipase C epsilon. Mol Cell 21, 495-507.

Bunney TD, Opaleye O, Roe SM, Vatter P, Baxendale RW, Walliser C, Everett KL, Josephs MB, Christow C, Rodrigues-Lima F, Gierschik P, Pearl LH \& Katan M (2009) Structural Insights into Formationof an Active Signaling Complex between Rac and Phospholipase C Gamma 2. Mol Cell 34, 223-233.

Burguete AS, Fenn TD, Brunger AT \& Pfeffer SR (2008) Rab and Arl GTPase Family Members Cooperate in the Localization of the Golgin GCC185. CELL 132, 286-298.

Chandra A, Grecco HE, Pisupati V, Perera D, Cassidy L, Skoulidis F, Ismail SA, Hedberg C, Hanzal-Bayer M \& Venkitaraman AR (2012) The GDI-like solubilizing factor PDE [delta] sustains the spatial organization and signalling of Ras family proteins. Nat Cell Biol 14, 148-158.

Chavas LMG, Ihara K, Kawasaki M, Torii S, Uejima T, Kato R, Izumi T \& Wakatsuki S (2008) Elucidation of Rab27 Recruitment by Its Effectors: Structure of Rab27a Bound to Exophilin4/Slp2-a. Structure 16, 1468-1477.

Cheng W, Yin K, Lu D, Li B, Zhu D, Chen Y, Zhang H, Xu S, Chai J \& Gu L (2012) Structural insights into a unique Legionella pneumophila effector LidA recognizing both GDP and GTP bound Rab1 in their active state. PLoS pathogens 
$8, \mathrm{e} 1002528$.

Cherfils J \& Zeghouf M (2013) Regulation of Small GTPases by GEFs, GAPs, and GDIs. Physiological Reviews 93, 269-309.

Chook Y \& Blobel G (1999) Structure of the nuclear transport complex karyopherinbeta 2- Ran center dot GppNHp. Nature 399, 230-237.

Collins BM, Watson PJ \& Owen DJ (2003) The structure of the GGA1-GAT domain reveals the molecular basis for ARF binding and membrane association of GGAs. Dev Cell 4, 321-332.

Cook A, Bono F, Jinek M \& Conti E (2007) Structural Biology of Nucleocytoplasmic Transport. Annu Rev Biochem 76, 647-671.

Cook A, Fernandez E, Lindner D, Ebert J, Schlenstedt G \& Conti E (2005) The Structure of the Nuclear Export Receptor Cse1 in Its Cytosolic State Reveals a Closed Conformation Incompatible with Cargo Binding. Mol Cell 18, 355-367.

Cook AG, Fukuhara N, Jinek M \& Conti E (2009) Structures of the tRNA export factor in the nuclear and cytosolic states. Nature 461, 60-65.

De Haan L \& Hirst TR (2004) Cholera toxin: a paradigm for multi-functional engagement of cellular mechanisms (Review). Mol. Membr. Biol. 21, 77-92.

de Vos AM, TONG L, MILBURN MV, Matias PM, Jancarik J, Noguchi S, Nishimura S, Miura K, Ohtsuka E \& Kim SH (1988) Three-dimensional structure of an oncogene protein: catalytic domain of human c-H-ras p21. Science 239, 888-893.

Depetris RS, Wu J \& Hubbard SR (2009) Structural and functional studies of the Rasassociating and pleckstrin-homology domains of Grb10 and Grb14. Nat Struct Mol Biol 16, 833-839.

DiNitto JP, Delprato A, Gabe Lee M-T, Cronin TC, Huang S, Guilherme A, Czech MP \& Lambright DG (2007) Structural basis and mechanism of autoregulation in 3-phosphoinositide-dependent Grp1 family Arf GTPase exchange factors. Mol Cell 28, 569-583.

Dubois T, Paléotti O, Mironov AA, Fraisier V, Stradal TEB, De Matteis MA, Franco M \& Chavrier P (2005) Golgi-localized GAP for Cdc42 functions downstream of ARF1 to control Arp2/3 complex and F-actin dynamics. Nat Cell Biol 7, 353364.

Dvorsky R, Blumenstein L, Vetter IR \& Ahmadian MR (2004) Structural insights into the interaction of ROCKI with the switch regions of RhoA.J Biol Chem 279, 7098-7104.

Eathiraj S, Mishra A, Prekeris R \& Lambright DG (2006) Structural Basis for Rab11mediated Recruitment of FIP3 to Recycling Endosomes. J Mol Biol 364, 121135. 
Eathiraj S, Pan X, Ritacco C \& Lambright DG (2005) Structural basis of family-wide Rab GTPase recognition by rabenosyn-5. Nature 436, 415-419.

Emerson SD, Madison V, Palermo R, Waugh D, Scheffler J, Tsao K, Kiefer S, Liu S \& Fry D (1995) Solution Structure of the Ras-Binding Domain of C-Raf-1 and Identification of Its Ras Interaction Surface. Biochemistry 34, 6911-6918.

Emerson SD, Waugh DS, Scheffler JE, Tsao KL, Prinzo KM \& Fry DC (1994) Chemical shift assignments and folding topology of the Ras-binding domain of human Raf-1 as determined by heteronuclear three-dimensional NMR spectroscopy. Biochemistry 33, 7745-7752.

Fenwick RB, Campbell LJ, Rajasekar K, Prasannan S, Nietlispach D, Camonis JH, Owen D \& Mott HR (2010) The RalB-RLIP76 complex reveals a novel mode of ral-effector interaction. Structure 18, 985-995.

Fenwick RB, Prasannan S, Campbell LJ, Nietlispach D, Evetts KA, Camonis JH, Mott HR \& Owen D (2009) Solution Structure and Dynamics of the Small GTPase RalB in Its Active Conformation: Significance for Effector Protein Binding. Biochemistry 48, 2192-2206.

Fried H \& Kutay U (2003) Nucleocytoplasmic transport: taking an inventory. Cellular and Molecular Life Sciences 60, 1659-1688.

Fukai S, Matern HT, Jagath JR, Scheller RH \& Brunger AT (2003) Structural basis of the interaction between RalA and Sec5, a subunit of the sec6/8 complex. EMBOJ $22,3267-3278$.

Garrard S, Capaldo C, Gao L, Rosen M, Macara I \& Tomchick D (2003) Structure of Cdc42 in a complex with the GTPase-binding domain of the cell polarity protein, Par6. Embo Journal 22, 1125-1133.

Gingras AR, Puzon-Mclaughlin W \& Ginsberg MH (2013) The Structure of the Ternary Complex of Krev Interaction Trapped 1 (KRIT1) Bound to Both the Rap1 GTPase and the Heart of Glass (HEG1) Cytoplasmic Tail. J Biol Chem 288, 23639-23649.

Glading A, Han J, Stockton RA \& Ginsberg MH (2007) KRIT-1/CCM1 is a Rap1 effector that regulates endothelial cell cell junctions. Journal of Cell Biology 179, $247-254$.

Gorman C, Skinner R, Skelly J, Neidle S \& Lowe P (1996) Equilibrium and kinetic measurements reveal rapidly reversible binding of ras to raf. J Biol Chem 271, 6713-6719.

GrUnwald M, Lazzaretti D \& Bono F (2013) Structural basis for the nuclear export activity of Importin13. EMBO J 32, 899-913.

Güttler T \& Görlich D (2011) Ran-dependent nuclear export mediators: a structural perspective. EMBO J 30, 3457-3474.

Hahn S \& Schlenstedt G (2011) Importin $\beta$-type nuclear transport receptors have 
distinct binding affinities for Ran-GTP. Biochem Biophys Res Commun 406, 383388 .

Hanzal-Bayer M, Renault L, Roversi P, Wittinghofer A \& Hillig RC (2002) The complex of Arl2-GTP and PDE delta: from structure to function. EMBO J 21, 2095-2106.

Heider MR \& Munson M (2012) Exorcising the Exocyst Complex. Traffic 13, 898907.

Herrmann CA, Horn G, Spaargaren M \& Wittinghofer A (1996) Differential interaction of the Ras family GTP-binding proteins H-Ras, Rap1A, and R-Ras with the putative effector molecules Raf kinase and Ral-guanine nucleotide exchange factor. J Biol Chem 271, 6794-6800.

Hesketh GG, Perez-Dorado I, Jackson LR, Wartosch L, Schaefer IB, Gray SR, McCoy AJ, Zeldin OB, Garman EF, Harbour ME, Evans PR, Seaman MNJ, Luzio JP \& Owen DJ (2014) VARP Is Recruited on to Endosomes by Direct Interaction with Retromer, Where Together They Function in Export to the Cell Surface. Dev Cell 29, 591-606.

Hoffman GR, Nassar N \& Cerione RA (2000) Structure of the Rho family GTPbinding protein $\mathrm{Cdc} 42$ in complex with the multifunctional regulator RhoGDI. CELL 100, 345-356.

Hota PK \& Buck M (2012) Plexin structures are coming: opportunities for multilevel investigations of semaphorin guidance receptors, their cell signaling mechanisms, and functions. Cellular and Molecular Life Sciences 69, 3765-3805.

Hou X, Hagemann N, Schoebel S, Blankenfeldt W, Goody RS, Erdmann KS \& Itzen A (2011) A structural basis for Lowe syndrome caused by mutations in the Rabbinding domain of OCRL1. EMBO J 30, 1659-1670.

Huang L, Hofer F, Martin GS \& Kim SH (1998) Structural basis for the interaction of Ras with RalGDS. Nature Structural Biology 5, 422-426.

Hutchinson CL, Lowe PN, McLaughlin SH, Mott HR \& Owen D (2013) Differential Binding of RhoA, RhoB, and RhoC to Protein Kinase C-Related Kinase (PRK) Isoforms PRK1, PRK2, and PRK3: PRKs Have the Highest Affinity for RhoB. Biochemistry 52, 7999-8011.

Hutchinson CL, Lowe PN, McLaughlin SH, Mott HR \& Owen D (2011) Mutational analysis reveals a single binding interface between RhoA and its effector, PRK1. Biochemistry 50, 2860-2869.

Isabet T, Montagnac G, Regazzoni K, Raynal B, Khadali El F, England P, Franco M, Chavrier P, Houdusse A \& Ménétrey J (2009) The structural basis of Arf effector specificity: the crystal structure of ARF6 in a complex with JIP4. EMBOJ 28, 2835-2845.

Ismail SA, Chen Y-X, Rusinova A, Chandra A, Bierbaum M, Gremer L, Triola G, Waldmann H, Bastiaens PIH \& Wittinghofer A (2011) Arl2-GTP and Arl3-GTP 
regulate a GDI-like transport system for farnesylated cargo. Nat Chem Biol 7, 942-949.

Jackson LP, Kelly BT, McCoy AJ, Gaffry T, James LC, Collins BM, Höning S, Evans PR \& Owen DJ (2010) A Large-Scale Conformational Change Couples Membrane Recruitment to Cargo Binding in the AP2 Clathrin Adaptor Complex. CELL 141, 1220-1229.

Jackson LP, Kümmel D, Reinisch KM \& Owen DJ (2012) Structures and mechanisms of vesicle coat components and multisubunit tethering complexes. Curr Opin Cell Biol 24, 475-483.

Jaffer ZM \& Chernoff J (2002) p21-activated kinases: three more join the Pak. Int J Biochem Cell Biol 34, 713-717.

Jagoe WN, Lindsay AJ, Read RJ, McCoy AJ, McCaffrey MW \& Khan AR (2006) Crystal Structure of Rab11 in Complex with Rab11 Family Interacting Protein 2. Structure 14, 1273-1283.

Jezyk M, Snyder J, Gershberg S, Worthylake D, Harden T \& Sondek J (2006) Crystal structure of Rac1 bound to its effector phospholipase C-beta 2. Nature Structural \& Molecular Biology 13, 1135-1140.

Jin R, Junutula JR, Matern HT, Ervin KE, Scheller RH \& Brunger AT (2005) Exo84 and Sec5 are competitive regulatory Sec6/8 effectors to the RalA GTPase. EMBO $J$ 24, 2064-2074.

Joberty G, Petersen C, Gao L \& Macara I (2000) The cell-polarity protein Par6 links Par3 and atypical protein kinase C to Cdc42. Nat Cell Biol 2, 531-539.

Kast DJ, Yang C, Disanza A, Boczkowska M, Madasu Y, Scita G, Svitkina T \& Dominguez R (2014) Mechanism of IRSp53 inhibition and combinatorial activation by Cdc42 and downstream effectors. Nat Struct Mol Biol 21, 413-422.

Kelley GG, Reks SE, Ondrako JM \& Smrcka AV (2001) Phospholipase C(epsilon): a novel Ras effector. EMBO J 20, 743-754.

Khan AR \& Ménétrey J (2013) Structural Biology of Arf and Rab GTPases' Effector Recruitment and Specificity. Structure/Folding and Design 21, 1284-1297.

Kukimoto-Niino M, Sakamoto A, Kanno E, Hanawa-Suetsugu K, Terada T, Shirouzu M, Fukuda M \& Yokoyama S (2008) Structural Basis for the Exclusive Specificity of Slac2-a/Melanophilin for the Rab27 GTPases. Structure 16, 14781490.

Lall P, Horgan CP, Oda S, Franklin E, Sultana A, Hanscom SR, McCaffrey MW \& Khan AR (2013) Structural and functional analysis of FIP2 binding to the endosome-localised Rab25 GTPase. Biochim Biophys Acta 1834, 2679-2690.

Lammers M, Meyer S, Kuhlmann D \& Wittinghofer A (2008) Specificity of Interactions between mDia Isoforms and Rho Proteins. J Biol Chem 283, 3523635246. 
Lapouge K, Smith SJ, Walker PA, Gamblin SJ, Smerdon SJ \& Rittinger K (2000) Structure of the TPR domain of p67phox in complex with Rac.GTP. Mol Cell 6, 899-907.

Lawe DC, Patki V, Heller-Harrison R, Lambright D \& Corvera S (2000) The FYVE Domain of Early Endosome Antigen 1 Is Required for Both Phosphatidylinositol 3-Phosphate and Rab5 Binding CRITICAL ROLE OF THIS DUAL INTERACTION FOR ENDOSOMAL LOCALIZATION. J Biol Chem 275, 3699-3705.

Lee SJ, Matsuura Y, Liu SM \& Stewart M (2005) Structural basis for nuclear import complex dissociation by RanGTP. Nature 435, 693-696.

Lei M, Lu W, Meng W, Parrini M, Eck M, Mayer B \& Harrison S (2000) Structure of PAK1 in an autoinhibited conformation reveals a multistage activation switch. CELL 102, 387-397.

Li X, Zhang R, Draheim KM, Liu W, Calderwood DA \& Boggon TJ (2012) Structural basis for the small G-protein-effector interaction of Ras-related protein 1 (Rap1) and the adaptor protein Krev interaction trapped 1 (KRIT1). J Biol Chem 287, 22317-22327.

Linnemann T, Geyer M, Jaitner B, Block C, Kalbitzer H, Wittinghofer A \& Herrmann CA (1999) Thermodynamic and kinetic characterization of the interaction between the Ras binding domain of AF6 and members of the Ras subfamily. $J$ Biol Chem 274, 13556-13562.

Linnemann T, Kiel C, Herter P \& Herrmann CA (2002) The activation of RalGDS can be achieved independently of its Ras binding domain. Implications of an activation mechanism in Ras effector specificity and signal distribution. $J$ Biol Chem 277, 7831-7837.

Lu L \& Hong W (2003) Interaction of Arl1-GTP with GRIP domains recruits autoantigens Golgin-97 and Golgin-245/p230 onto the Golgi. Molecular Biology of the Cell 14, 3767-3781.

Lu L, Tai G, Wu M, Song H \& Hong W (2006) Multilayer Interactions Determine the Golgi Localization of GRIP Golgins. Traffic 7, 1399-1407.

Maesaki R, Ihara K, Shimizu T, Kuroda S, Kaibuchi K \& Hakoshima T (1999) The structural basis of Rho effector recognition revealed by the crystal structure of human RhoA complexed with the effector domain of PKN/PRK1. Mol Cell 4, 793-803.

Makyio H, Ohgi M, Takei T, Takahashi S, Takatsu H, Katoh Y, Hanai A, Ueda T, Kanaho Y, Xie Y, Shin H-W, Kamikubo H, Kataoka M, Kawasaki M, Kato R, Wakatsuki S \& Nakayama K (2012) Structural basis for Arf6-MKLP1 complex formation on the Flemming body responsible for cytokinesis. EMBO J 31, 25902603.

Malaby AW, van den Berg B \& Lambright DG (2013) Structural basis for membrane recruitment and allosteric activation of cytohesin family Arf GTPase exchange 
factors. Proc Natl Acad Sci USA 110, 14213-14218.

Margarit S, Sondermann H, Hall BE, Nagar B, Hoelz A, Pirruccello M, Bar-Sagi D \& Kuriyan J (2003) Structural evidence for feedback activation by Ras· GTP of the Ras-specific nucleotide exchange factor SOS. CELL 112, 685-695.

Matsuura Y \& Stewart M (2004) Structural basis for the assembly of a nuclear export complex. Nature 432, 872-877.

Mcmahon HT \& Mills IG (2004) COP and clathrin-coated vesicle budding: different pathways, common approaches. Curr Opin Cell Biol 16, 379-391.

Ménétrey J, Perderiset M, Cicolari J, Dubois T, Elkhatib N, Khadali El F, Franco M, Chavrier P \& Houdusse A (2007) Structural basis for ARF1-mediated recruitment of ARHGAP21 to Golgi membranes. EMBO J 26, 1953-1962.

Miki H, Yamaguchi H, Suetsugu S \& Takenawa T (2000) IRSp53 is an essential intermediate between Rac and WAVE in the regulation of membrane ruffling. Nature 408, 732-735.

Milburn MV, Tong L, Devos AM, Brunger A, Yamaizumi Z, Nishimura S \& Kim SH (1990) Molecular switch for signal transduction: structural differences between active and inactive forms of protooncogenic ras proteins. Science 247, 939-945.

Mishra A, Eathiraj S, Corvera S \& Lambright DG (2010) Structural basis for Rab GTPase recognition and endosome tethering by the $\mathrm{C} 2 \mathrm{H} 2$ zinc finger of Early Endosomal Autoantigen 1 (EEA1). Proceedings of the National Academy of Sciences 107, 10866-10871.

Mizuno-Yamasaki E, Rivera-Molina F \& Novick P (2012) GTPase Networks in Membrane Traffic. Annu Rev Biochem 81, 637-659.

Modha R, Campbell LJ, Nietlispach D, Buhecha HR, Owen D \& Mott HR (2008) The Rac1 polybasic region is required for interaction with its effector PRK1. J Biol Chem 283, 1492-1500.

Monecke T, Guttler T, Neumann P, Dickmanns A, Gorlich D \& Ficner R (2009) Crystal Structure of the Nuclear Export Receptor CRM1 in Complex with Snurportin1 and RanGTP. Science 324, 1087-1091.

Morreale A, Venkatesan M, Mott HR, Owen D, Nietlispach D, Lowe P \& Laue E (2000) Structure of Cdc42 bound to the GTPase binding domain of PAK. Nature Structural Biology 7, 384-388.

Mott HR, Nietlispach D, Hopkins LJ, Mirey G, Camonis JH \& Owen D (2003) Structure of the GTPase-binding domain of Sec5 and elucidation of its Ral binding site. J Biol Chem 278, 17053-17059.

Mott HR, Owen D, Nietlispach D, Lowe PN, Manser E, Lim L \& Laue ED (1999) Structure of the small $\mathrm{G}$ protein Cdc42 bound to the GTPase-binding domain of ACK. Nature 399, 384-388. 
Muromoto R, Sekine Y, Imoto S, Ikeda O, Okayama T, Sato N \& Matsuda T (2008) BART is essential for nuclear retention of STAT3. Int Immunol 20, 395-403.

Nakamura K, Man Z, Xie Y, Hanai A, Makyio H, Kawasaki M, Kato R, Shin H-W, Nakayama K \& Wakatsuki S (2012) Structural Basis for Membrane Binding Specificity of the Bin/Amphiphysin/Rvs (BAR) Domain of Arfaptin-2 Determined by Arl1 GTPase. J Biol Chem 287, 25478-25489.

Nassar N, Horn G, Herrmann CA, Block C, Janknecht R \& Wittinghofer A (1996) Ras/Rap effector specificity determined by charge reversal. Nature Structural Biology 3, 723-729.

Nassar N, Horn G, Herrmann CA, Scherer A, McCormick F \& Wittinghofer A (1995) The $2.2 \AA$ crystal structure of the Ras-binding domain of the serine/threonine kinase c-Raf1 in complex with RaplA and a GTP analogue. Nature 375, 554-560.

O'Neal CJ, Amaya EI, Jobling MG, Holmes RK \& Hol W (2004) Crystal structures of an intrinsically active cholera toxin mutant yield insight into the toxin activation mechanism. Biochemistry 43, 3772-3782.

O'Neal CJ, Jobling MG, Holmes RK \& Hol W (2005) Structural basis for the activation of cholera toxin by human ARF6-GTP. Science 309, 1093-1096.

Okada C, Yamashita E, Lee SJ, Shibata S, Katahira J, Nakagawa A, Yoneda Y \& Tsukihara T (2009) A High-Resolution Structure of the Pre-microRNA Nuclear Export Machinery. Science 326, 1275-1279.

Ostermeier C \& Brunger A (1999) Structural basis of Rab effector specificity: Crystal structure of the small $\mathrm{G}$ protein Rab3A complexed with the effector domain of Rabphilin-3A. CELL 96, 363-374.

Owen D, Lowe P, Nietlispach D, Brosnan C, Chirgadze D, Parker P, Blundell T \& Mott HR (2003) Molecular dissection of the interaction between the small G proteins Rac1 and RhoA and protein kinase C-related kinase 1 (PRK1). J Biol Chem 278, 50578-50587.

Owen D, Mott HR, Laue E \& Lowe P (2000) Residues in Cdc42 that specify binding to individual CRIB effector proteins. Biochemistry 39, 1243-1250.

Pacold ME, Suire S, Perisic O, Lara-Gonzalez S, Davis CT, Walker EH, Hawkins PT, Stephens L, Eccleston JF \& Williams RL (2000) Crystal structure and functional analysis of Ras binding to its effector phosphoinositide 3-kinase $\gamma$. CELL 103, 931-944.

Pai E, Kabsch W, Krengel U, Holmes K, John J \& Wittinghofer A (1989) Structure of the Guanine-Nucleotide-Binding Domain of the Ha- Ras Oncogene Product P21 in the Triphosphate Conformation. Nature 341, 209-214.

Pai EF, Krengel U, PETSKO GA, Goody RS, Kabsch W \& Wittinghofer A (1990) Refined Crystal-Structure of the Triphosphate Conformation of H-Ras P21 at 1.35 a Resolution - Implications for the Mechanism of Gtp Hydrolysis. EMBO J 9, 2351-2359. 
Panic B, Perisic O, Veprintsev DB, Williams RL \& Munro S (2003) Structural basis for Arl1-dependent targeting of homodimeric GRIP domains to the Golgi apparatus. Mol Cell 12, 863-874.

Pirruccello M \& Pietro De Camilli (2012) Inositol 5-phosphatases: insights fromthe Lowe syndrome protein OCRL. Trends Biochem Sci 37, 134-143.

Price HP, Peltan A, Stark M \& Smith DF (2010) The small GTPase ARL2 is required for cytokinesis in Trypanosoma brucei. Mol Biochem Parasitol 173, 123-131.

Pylypenko O, Attanda W, Gauquelin C, Lahmani M, Coulibaly D, Baron B, Hoos S, Titus MA, England P \& Houdusse AM (2013) Structural basis of myosin V Rab GTPase-dependent cargo recognition. Proc Natl Acad Sci USA 110, 2044320448.

Qamra R \& Hubbard SR (2013) Structural Basis for the Interaction of the Adaptor Protein Grb14 with Activated Ras W. Xu, ed. PLoS ONE 8, e72473.

Ranganathan R \& Ross EM (1997) PDZ domain proteins: scaffolds for signaling complexes. Current Biology 7, R770-3.

Recacha R, Boulet A, Jollivet F, Monier S, Houdusse A, Goud B \& Khan AR (2009) Structural Basis for Recruitmentof Rab6-Interacting Protein 1 to Golgi via a RUN Domain. Structure/Folding and Design 17, 21-30.

Ren X, Farías GG, Canagarajah BJ, Bonifacino JS \& Hurley JH (2013) Structural Basis for Recruitment and Activation of the AP-1 Clathrin Adaptor Complex by Arf1. CELL 152, 755-767.

Rodriguez-Viciana P, Warne PH, Khwaja A, Marte BM, Pappin D, Das P, Waterfield MD, Ridley A \& Downward J (1997) Role of phosphoinositide 3-OH kinase in cell transformation and control of the actin cytoskeleton by Ras. CELL 89, 457467.

Rojas AM, Fuentes G, Rausell A \& Valencia A (2012) The Ras protein superfamily: evolutionary tree and role of conserved amino acids. Journal of Cell Biology 196, 189-201.

Rose R, Weyand M, Lammers M, Ishizaki T, Ahmadian MR \& Wittinghofer A (2005) Structural and mechanistic insights into the interaction between Rho and mammalian Dia. Nature 435, 513-518.

Sahai E, Alberts AS \& Treisman R (1998) RhoA effector mutants reveal distinct effector pathways for cytoskeletal reorganization, SRF activation and transformation. EMBOJ 17, 1350-1361.

Schaefer IB, Hesketh GG, Bright NA, Gray SR, Pryor PR, Evans PR, Luzio JP \& Owen DJ (2012) The binding of Varp to VAMP7 traps VAMP7 in a closed, fusogenically inactive conformation. Nature Structural \& Molecular Biology 19, 1300-.

Scheffzek K \& Welti S (2012) Pleckstrin homology (PH) like domains - versatile 
modules in protein-protein interaction platforms. FEBS Lett 586, 2662-2673.

Scheffzek K, Grunewald P, Wohlgemuth S, Kabsch W, Tu H, Wigler MH, Wittinghofer A \& Herrmann CA (2001) The Ras-Byr2RBD complex: Structural basis for Ras effector recognition in yeast. Structure/Folding and Design 9, 10431050 .

Schoebel S, Cichy AL, Goody RS \& Itzen A (2011) Protein LidA from Legionella is a Rab GTPase supereffector. Proc Natl Acad Sci USA 108, 17945-17950.

Sharer JD, Shern JF, Van Valkenburgh H, Wallace DC \& Kahn RA (2002) ARL2 and BART enter mitochondria and bind the adenine nucleotide transporter. Molecular Biology of the Cell 13, 71-83.

Shiba T, Kawasaki M, Takatsu H, Nogi T, Matsugaki N, Igarashi N, Suzuki M, Kato R, Nakayama K \& Wakatsuki S (2003) Molecular mechanism of membrane recruitment of GGA by ARF in lysosomal protein transport. Nature Structural Biology 10, 386-393.

Shiba T, Koga H, Shin H-W, Kawasaki M, Kato R, Nakayama K \& Wakatsuki S (2006) Structural basis for Rab11-dependent membrane recruitment of a family of Rab11-interacting protein 3 (FIP3)/Arfophilin-1. Proc Natl Acad Sci USA 103, 15416-15421.

Siebold C \& Jones EY (2013) Seminars in Cell \& Developmental Biology. Semin Cell Dev Biol 24, 139-145.

Snyder JT, Singer AU, Wing MR, Harden TK \& Sondek J (2003) The Pleckstrin Homology Domain of Phospholipase C- 2 as an Effector Site for Rac. J Biol Chem 278, 21099-21104.

Stalder D \& Antonny B (2013) Arf GTPase regulation through cascade mechanisms and positive feedback loops. FEBS Lett 587, 2028-2035.

Stieglitz B, Bee C, Schwarz D, Yildiz O, Moshnikova A, Khokhlatchev A \& Herrmann CA (2008) Novel type of Ras effector interaction established between tumour suppressor NORE1A and Ras switch II. EMBO J 27, 1995-2005.

Suer S, Misra S, Saidi LF \& Hurley JH (2003) Structure of the GAT domain of human GGA1: A syntaxin amino-terminal domain fold in an endosomal trafficking adaptor. Proc Natl Acad Sci USA 100, 4451-4456.

Sydor J, Engelhard M, Wittinghofer A, Goody R \& Herrmann CA (1998) Transient kinetic studies on the interaction of ras and the Ras-binding domain of c-Raf-1 reveal rapid equilibration of the complex. Biochemistry 37, 14292-14299.

Takai Y, Sasaki T \& Matozaki T (2001) Small GTP-binding proteins. Physiological Reviews 81, 153-208.

Tarricone C, Xiao B, Justin N, Walker P, Rittinger K, Gamblin S \& Smerdon S (2001) The structural basis of Arfaptin-mediated cross-talk between Rac and Arf signalling pathways. Nature 411, 215-219. 
Tong Y, Chugha P, Hota PK, Alviani RS, Li M, Tempel W, Shen L, Park H-W \& Buck M (2007) Binding of Rac1, Rnd1, and RhoD to a Novel Rho GTPase Interaction Motif Destabilizes Dimerization of the Plexin-B1 Effector Domain. $J$ Biol Chem 282, 37215-37224.

Tong Y, Hota PK, Penachioni JY, Hamaneh MB, Kim S, Alviani RS, Shen L, He H, Tempel W, Tamagnone L, Park H-W \& Buck M (2009) Structure and Function of the Intracellular Region of the Plexin-B1 Transmembrane Receptor. J Biol Chem 284, 35962-35972.

Vetter I \& Wittinghofer A (2001) Signal transduction - The guanine nucleotidebinding switch in three dimensions. Science 294, 1299-1304.

Vetter I, Linnemann T, Wohlgemuth S, Geyer M, Kalbitzer H, Herrmann CA \& Wittinghofer A (1999a) Structural and biochemical analysis of Ras-effector signaling via RalGDS. FEBS Lett 451, 175-180.

Vetter IR, Arndt A, Kutay U, Gorlich D \& Wittinghofer A (1999b) Structural view of the Ran-importin beta interaction at 2.3 angstrom resolution. CELL 97, 635-646.

Vetter IR, Nowak C, Nishimoto T, Kuhlmann J \& Wittinghofer A (1999c) Structure of a Ran-binding domain complexed with Ran bound to a GTP analogue: implications for nuclear transport. Nature 398, 39-46.

Vojtek AB, Hollenberg SM \& Cooper JA (1993) Mammalian Ras interacts directly with the serine/threonine kinase Raf. CELL 74, 205-214.

Walliser C, Retlich M, Harris R, Everett KL, Josephs MB, Vatter P, Esposito D, Driscoll PC, Katan M, Gierschik P \& Bunney TD (2008) Rac Regulates Its Effector Phospholipase $\mathrm{C} 2$ through Interaction with a Split Pleckstrin Homology Domain. J Biol Chem 283, 30351-30362.

Wang H, Hota PK, Tong Y, Li B, Shen L, Nedyalkova L, Borthakur S, Kim S, Tempel W, Buck M \& Park H-W (2011) Structural Basis of Rnd1 Binding to Plexin Rho GTPase Binding Domains (RBDs). J Biol Chem 286, 26093-26106.

Wang Y, He H, Srivastava N, Vikarunnessa S, Chen Y-B, Jiang J, Cowan CW \& Zhang X (2012) Plexins are GTPase-activating proteins for Rap and are activated by induced dimerization. Science Signaling 5, ra6.

Wei J, Fain S, Harrison C, Feig LA \& Baleja JD (2006) Molecular Dissection of Rab11 Binding from Coiled-Coil Formation in the Rab11-FIP2 C-Terminal Domain $\uparrow$. Biochemistry 45, 6826-6834.

White MA (1995) Multiple ras functions can contribute to mammalian cell transformation. CELL 80, 533-541.

Wu M, Lu L, Hong W \& Song H (2004) Structural basis for recruitment of GRIP domain golgin-245 by small GTPase Arl1. Nature Structural \& Molecular Biology 11, 86-94.

Wu MS, Wang TL, Loh E, Hong WJ \& Song HW (2005) Structural basis for 
recruitment of RILP by small GTPase Rab7. EMBO J 24, 1491-1501.

Wynne JP, Wu J, Su W, Mor A, Patsoukis N, Boussiotis VA, Hubbard SR \& Philips MR (2012) Rap1-interacting adapter molecule (RIAM) associates with the plasma membrane via a proximity detector. The Journal of Cell Biology 199, 317-330.

Yamashita M, Kurokawa K, Sato Y, Yamagata A, Mimura H, Yoshikawa A, Sato K, Nakano A \& Fukai S (2010) Structural basis for the Rho-and phosphoinositidedependent localization of the exocyst subunit Sec3. Nature Structural \& Molecular Biology 17, 180-186.

Yu X, Breitman M \& Goldberg J (2012) A structure-based mechanism for Arf1dependent recruitment of coatomer to membranes. CELL 148, 530-542.

Zhang H, Chang Y-C, Brennan ML \& Wu J (2014a) The structure of Rap1 in complex with RIAM reveals specificity determinants and recruitment mechanism. J Mol Cell Biol 6, 128-139.

Zhang RG, Scott DL, Westbrook ML, Nance S, Spangler BD, Shipley GG \& Westbrook EM (1995) The three-dimensional crystal structure of cholera toxin. $J$ Mol Biol 251, 563-573.

Zhang T, Li S, Zhang Y, Zhong C, Lai Z \& Ding J (2009) Crystal structure of the ARL2-GTP-BART complex reveals a novel recognition and binding mode of small GTPase with effector. Structure/Folding and Design 17, 602-610.

Zhang X, He X, Fu X-Y \& Chang Z (2006) Varp is a Rab21 guanine nucleotide exchange factor and regulates endosome dynamics. J Cell Sci 119, 1053-1062.

Zhang Z, Zhang T, Wang S, Gong Z, Tang C, Chen J \& Ding J (2014b) Molecular mechanism for Rabex-5 GEF activation by Rabaptin-5. eLife 3, e02687-e02687.

Zhao Z, Manser E, Chen X, Chong C, Leung T \& Lim L (1998) A conserved negative regulatory region in alpha PAK: Inhibition of PAK kinases reveals their morphological roles downstream of Cdc42 and Rac1. Mol Cell Biol 18, 21532163.

Zhu G, Zhai P, He X, Terzyan S, Zhang R, Joachimiak A, Tang J \& Zhang XC (2003) Crystal structure of the human GGA1 GAT domain. Biochemistry 42, 6392-6399.

Zhu G, Zhai P, Liu J, Terzyan S, Li G \& Zhang XC (2004) Structural basis of Rab5Rabaptin5 interaction in endocytosis. Nat Struct Mol Biol 11, 975-983. 


\section{Figure Legends}

Figure 1.1. Structures of H-Ras.
A. Ras·GDP (1AA9)
B. Ras·GMPPCP (121P)
C. Topology representation of the Ras protein.

Helices are coloured pale yellow, strands are aquamarine and loops are grey. Switch 1 and switch 2 are coloured pink. The orientation and colour scheme are the 'standard' ones used in all subsequent figures unless stated. The nucleotide is shown in a stick representation, with atoms coloured as follows: $\mathrm{C}=$ green, $\mathrm{N}=$ blue, $\mathrm{O}=\mathrm{red}, \mathrm{P}=$ orange. The $\mathrm{Mg}^{2+}$ ion is shown as a pale pink sphere.

Figure 1.2. Structures of representatives from the Rho, Arf, Rab and Ran families in their active (GTP-bound) and inactive (GDP-bound) states.

The colours and orientations are the same as in Figure 1.1. The nucleotide is shown but the $\mathrm{Mg}^{2+}$ ion has been omitted so that the nucleotide is clearly visible.

A. RhoA: left GDP (1FTN), right GTP $\gamma \mathrm{S}$ (1A2B). Rho family proteins contain an extra sequence between $\beta 5$ and $\alpha 4$, known as the 'insert region' which forms a single turn helix, followed by an $\alpha$-helix at the top in this orientation.

B. Arf6: left GDP (1E0S), right GTP $\gamma$ S (2J5X). Arf family proteins have an extra helix at the N-terminus, which is visible in the GDP-bound form as it packs against the rest of the $\mathrm{G}$ domain but removed in the construct used for the GTP-bound structure due to crystallization constraints. There is also an extra $\beta$-strand formed by switch 1 in the GDP-bound form, which extends the $\beta$-sheet next to $\beta 2$.

C. Rab7: left GDP (1VG1), right GMPPNP (1VG8). Rab proteins do not have any extra structural features compared to Ras. The GDP-bound form is missing parts of both switch 1 and switch 2, because they are flexible and therefore invisible in the Xray structure.

D. Ran: left GDP (3GJ0), right GMPPNP (1RRP). The Ran protein has a C-terminal extension that forms an extra $\alpha$-helix and is behind the G domain in the GDP-bound form in this orientation. In the GDP-bound form there is an extra turn of $\alpha$-helix within switch 1 and also a short section of $\beta$-strand in switch 1 , which extends the $\beta$ - 
sheet next to $\beta 2$. The GMPPNP structure shown is from the RanBP2 complex as there is no free GMPPNP structure.

Figure 2.1. The structures of H-Ras and Rap1 with ubiquitin-like fold effector domains.

A. H-Ras in complex with c-Raf1 (4G0N) in the standard orientation from Figure 1.1. The Ras protein is in the same colours as Figure 1.1 (the standard colour scheme) and the Raf protein is coloured purple.

B-F. All of the complexes are oriented so that the intermolecular $\beta$-sheet can be seen more clearly. The $\mathrm{G}$ protein is in the same orientation in each panel and is shown in the standard colour scheme. The effector proteins are shown in purple.

B. H-Ras in complex with cRaf1 (4G0N)

C. H-Ras in complex with RalGDS (1LFD)

D. H-Ras in complex with PI3K (1HE8)

E. H-Ras in complex with NORE1 (3DDC).

F. Rap1B in complex with KRIT1 (4HDO). The KRIT1 domains are coloured as follows: purple (F1), mid-blue (F2) and light blue (F3). Note that parts of both switch 1 and switch 2 were missing in this structure.

Figure 2.2.The electrostatic surfaces of Ras and Raf are complementary.

The surfaces are shown in an 'open book' form, with the interacting surfaces facing the viewer. Charged residues on the two molecules are labelled. The salt bridges that are discussed in the text are depicted by yellow dotted lines.

Figure 2.3. The structure of RalA in complex with exocyst complex components. The two complex structures are overlaid over the RalA structure. The RalA proteins in the complexes are in the same orientation as in Figure 2.1, so that the intermolecular $\beta$-sheets are visible.

A. RalA with Sec5 (1UAD). RalA is shown in the standard colour scheme and the Sec5 domain is purple.

B. RalA with Exo84 (1ZC3). RalA is shown in the standard colour scheme and the Exo84 domain is purple. 
Figure 2.4. The structure of Cdc 42 in complex with CRIB containing effectors. The complexes were overlaid over $\mathrm{Cdc} 42$, which is oriented as in Figure 1.1 . Cdc42 is shown in the standard colour scheme and the effector proteins are all coloured purple.
A. Cdc42 with ACK (1CF4)
B. Cdc42 with WASP (1CEE)
C. Cdc42 with PAK1 (1E0A)

Figure 2.5.The structure of Cdc42 in complex with non-canonical CRIB effectors. The structures were overlaid over $\mathrm{Cdc} 42$ and oriented so that the intermolecular $\beta$ sheet is visible. Cdc42 is shown in the standard colour scheme and the effector proteins are coloured purple.
A. Cdc42 with PAR6 (1NF3)
B. Cdc42 with IRSp53 (4JSO)

Figure 2.6. The structure of Arl proteins in complex with effectors.

The Arl proteins are shown in standard colours and oriented as in Figure 2.1 so that the intermolecular $\beta$-sheet is visible. The effectors are coloured purple.
A. Arl2 in complex with PDE (1KSG).
B. Arl3 in complex with UNC119 (4GOJ).

Figure 2.7. The structure of Arf6 in complex with the MKLP1 dimer (3VHX). Arf6 is shown in the standard colours and oriented so that it is possible to see the intermolecular $\beta$-sheet in the monomer shown on the left, as well as the dimer interface. The intermolecular $\beta$-sheet in the complex on the right is obscured. The MKLP1 monomers are coloured purple and green. 
Figure 3.1. Cartoon representation of the six classes of effector proteins that bind using a pair of $\alpha$-helices to contact the G domain.

The four anti-parallel classes (panels B-E) are grouped according to the position of the loop between the helices (left or right) and whether the N-terminal helix (green) or C-terminal helix (purple) is on top when the structure is viewed with the G protein in this orientation. The two parallel classes (panels F and G) are grouped depending on whether the $\mathrm{N}$-terminus or C-terminus is on the left in this orientation. In the $\mathrm{G}$ domain cartoon (panel A) the $\alpha$-helices are coloured yellow, the $\beta$-strands are aquamarine, the loops are grey and the two switch regions are coloured pink.

Figure 3.2. The only Type B representative structure. The colours and orientation are the same as in Figure 3.1B.

RalB in complex with the coiled-coil from RLIP76(RalBP1) (2KWI).

Figure 3.3. Type $\mathrm{C}$ representative structures. The colours and orientation are the same as in Figure 3.1C.
A. Arf1 in complex with the GAT domain from GGA1 (1J2J).
B. Rab11 in complex with Myo5B globular tail domain (4LX0).
C. Rab8 in complex with the LidA super-effector from Legionella (3TNF).

Figure 3.4. Alternative views of Type $\mathrm{C}$ structures showing the entire effector molecule in the structures. The Rab proteins are in the same orientation as each other and shown in the standard colour scheme. The two helices of the helical pair that make most interactions are coloured as in Figure 3.1C.

A. Rab11-Myo5B. Helices 8 and 9 form the helical pair but some contacts are also made by other regions of Myo5B (coloured orange)

B. Rab8-LidA. Helices H4 and H5 form the helical pair. Extensive contacts are also made by other regions of LidA (coloured orange), which bind to several areas of Rab8. The base of the platform is formed by helices H1, H4, H5 and H7, which supports two pillars, pillar I (red dashed line) comprises helices H2 and $\mathrm{H} 3$, while pillar II (blue dashed line) includes H6 and the $\beta$-sheet. 
Figure 3.5. Type D representative structures.
A. Rab3A in complex with Exophilin1 (1ZBD)
B. Rab7 with RILP (1YHN)
C. Rab4 with Rabenosyn5 (1ZOK)
D. Arl1 with the Golgin245 GRIP domain (1UPT)
E. Rac1 with the HR1b domain from PRK1 (1RMK)
F. RhoC with mDial (1Z2C)
The colours and orientations are the same as in Figure 1D.

Figure 3.6. Structural differences in the RBD27 domains from Exophilin1, Exophilin 4 and Melanophilin. The colour schemes and orientations are the same as in Figure 3.1 .
A. The Exophilin1 helices are connected by a $\mathrm{Zn}^{2+}$ binding domain (coloured orange, $\mathrm{Zn}^{2+}$ is represented by a pink sphere).
B. The Exophilin4 helices are connected by a simple short loop.
C. The Melanophilin helices are connected by another type of $\mathrm{Zn}^{2+}$ binding domain (coloured orange, $\mathrm{Zn}^{2+}$ is a pink sphere).

Figure 3.7. The RhoC/mDia structure.

The two interacting helices are stabilized by a third helix, which packs behind them (dark orange). The colour scheme is the same as in Figure 3.1. The remainder of the $\mathrm{mDia}$ protein in the monomer interacting with $\mathrm{RhoC}$ is pale orange. Only one monomer of mDia is shown.

Figure 3.8. Type E representative structure.

A. Arl1 with Arfaptin2 (4DCN). The colours and orientation are as in Figure 3.1.

B. The Arl1 molecules bind on the convex face of the BAR domain dimer, leaving the membrane binding region of Arfaptin (concave surface, at the bottom of the crescent) free. The second Arfaptin chain is coloured pale orange. The two helices that contact one Arl1 molecule are coloured as in Figure 3.1 and the third helix in the same monomer is coloured dark orange.

C. Rac1 binding to Arfaptin1 on the concave surface of the BAR domain. Rac1 is coloured as in Figure 3.1. The Arfaptin helices are coloured as in B and shown in the same orientation. 
Figure 3.9.

A. Type F representative structures.Ras/Sos (1NVV)

B. RhoA/ROCK1 (1S1C)

C. Arf1/COP (3TJZ)

D. Arf6/JIP4 (2W83)

E. Rab11/FIP3 (2D7C)

F. Rab5/Rabaptin5 (1TU3)

G. Rab32/VARP (4CYM)

The colours and orientations are the same as in Figure 3.1. In B and D, the extra helix that connects the Type $\mathrm{F}$ helical pair is shown in orange. In $\mathrm{E}$ and $\mathrm{F}$, the extra short helices in the FIP3 and Rabaptin molecules are also shown in orange.

Figure 3.10. The allosteric modulation of Sos by Ras.

The Ras bound at the allosteric site (colours as in Figure 3.1) contacts the base of the helical hairpin (purple) that inserts into Ras. Ras at the active site is shown in dark red, the helical pair of Type $\mathrm{E}$ in Sos are shown in green, the helix between them is dark orange and the rest of Sos is coloured pale orange.

Figure 3.11. Interactions of Arf1 with tetrameric complexes from COP1 and AP1. The Arf1 molecules are in the same orientation in both panels.

A. Arf1 in complex with $\gamma \zeta$ from COP1. Arf1 is shown in the same colours as Figure 3.1. The helical pair of Type $\mathrm{E}$ is green. The remainder of the $\gamma$-COP subunit is orange and $\zeta$-COP is blue.

B. Arf1 in complex with the AP1 $\gamma-\beta 1-\mu 1-\sigma 1$ tetramer. Arf1 is shown in the same colours as in Figure 3.1. The helical pair of Type $\mathrm{E}$ is green. The remainder of the $\beta 1$ subunit is orange, the $\gamma$ subunit is dark pink, $\mu 1$ is blue and $\sigma 1$ is pale pink. The $\gamma$ subunit from the neighbouring monomer is also shown and is coloured dark red.

Figure 3.12. Interactions of Rab32 with VARP. 
A. The Rab32-binding helices in VARP are contained within 5 ankyrin repeats. Repeats 2 and 3 form the major contacts, although repeat 1 also forms a salt bridge. The Rab32 and VARP are coloured as in Figure 3.1 and 3.9 and oriented so that the interactions are visible.

B. The VARP ankyrin repeat domain forms a homodimer, forming a second interface between Rab32 and VARP (dashed ellipse). The colours are the same as in A but the second VARP molecule is coloured light orange.

Figure 3.13. Type $\mathrm{G}$ representative structures.

A. Rab6 in complex with Golgin GCC185 (3BBP).

B. Rab6 in complex with Rab6 interacting protein 1, Rab6IP1 (3CWZ).

The colours and orientations are the same as those in Figure 3.1G.

Figure 3.14. The Rab6/Rab6IP1 complex.

The helical RUN domain is shown in orange, except for the Rab-binding helices, which are purple. The PLAT domain is green.

Figure 4.1. The Rac1/p67 ${ }^{\text {phox }}$ complex (1E96).

Rac1 is shown in the same orientation and colour scheme as in Figure 1.2. The TPR repeats are coloured from dark blue to pale blue from the $\mathrm{N}$ - to the $\mathrm{C}$-terminal repeats. The insertion between repeats three and four is shown in orange and the C-terminal helix and extended region are shown in green.

Figure 4.2 The Rac1/plexinB1 complex (3SU8 and 3SUA).

A. Rac 1 is shown in the same orientation and colour scheme as in Figure 1.2. The Rac binding domain of plexinB1 is coloured purple and the RashoGAP domain into which it is inserted is coloured orange.

B. The structure of the isolated RBD from plexin B1 in complex with Rnd1. The Rnd1 is shown in the same colour scheme as Rac1 in A. The RBD is coloured purple. In B-D, the structures were overlaid over the Rho family protein whose switch regions are labelled and which makes contacts with $\mathrm{RBD}_{1}$.

C. The structure of the full intraceullular domain from plexin A1 in complex with Rac1. The Rac1 and plexin are shown in the same colours as in A.

URL: http:/mc.manuscriptcentral.com/\$B 
D. A second plexinB1 monomer in the trimer contacts Rac1 in the structure of the complex with the full intracellular domain of plexinB1,. The Rac1 and plexinB1 are shown in the same colours as in A. The switch regions for one of the Rac1 molecules are labelled to illustrate the contacts with the two plexin molecules..

Figure 4.3. The Arl2/BART complex (3DOE).

A. Arl2 is shown in the same orientation and colour scheme as in Figure 1.2. BART is shown in purple. The N-terminal helix of Arl2 is labelled $\alpha \mathrm{N}$ and the six helices of BART are labelled

B. As A but rotated through $180^{\circ}$ so that the interactions with the N-terminal $\alpha$ helix of Arl2 are apparent.

Figure 4.4 The Arf6/CTA1 complex (2A5D)

A. Arf6 is in the same orientation and colour scheme as in Figure 1.2 The CTA1 protein is shown in green.

B. As A but rotated so that the interactions with the CTA1 are apparent.

Figure 4.5. The Rab5/EEA1 complex (3MJH).

Rab5 is shown in the same orientation and colour scheme as in Figure 1.2. The EEA1 Rab binding domain is shown in purple, with its bound $\mathrm{Zn}^{2+}$ ion depicted as a green sphere.

Figure 4.6. The Rab8/OCRL complex (3QBT).

A. Rab8 is shown in the same orientation and colour scheme as in Figure 1.2. The OCRL ASH domain is coloured purple and the helical extension at the Nterminus is green.

B. As A but rotated to show the interactions with the two parts of the OCRL Rab binding fragment. Elements of Rab8 that interact with OCRL are labelled.

Figure 4.7. Ran effector complexes. 
A. The Ran/Importin complex (2BKU). Ran is in the same orientation and colour scheme as in Figure 1.2. The importin helices are shown in purple and the loops between them are green.

B. The same Ran/importin complex oriented so that the contacts with Ran can be seen clearly. The importin is coloured from blue (N-terminus) to red (Cterminus) and the Ran is coloured pink and purple for clarity. The switch regions are black.

C. The Ran/Cse1p/Kap60p complex (1WA5). The colour scheme and Ran orientation are the same as in A. Now the cargo is also present (Kap60p) and is shown in orange.

D. The same Ran/exportin complex with the Ran in the same orientation as in B. The colour scheme is the same as in B. The cargo is pale grey.

Figure 5.1. Effectors that interact via a $\mathrm{PH}$ domain.
A. RalA/Exo84 (1ZC3)
B. $\operatorname{Rac} 1 / \mathrm{PLC} \beta 2(2 \mathrm{FJU})$
C. $\operatorname{Rac} 2 / \mathrm{PLC} \gamma 2(2 \mathrm{~W} 2 \mathrm{X})$
D. Rho1p/Sec3p (3A58)
E. Arf6/Grp1 (4KAX)
F. Arf1/ARHGAP21 (2J59)
G. Ran/RanBP2 (1RRP)

In each panel the $\mathrm{G}$ protein is in the same orientation and colour scheme as in Figure 1.2, while the $\mathrm{PH}$ domain is shown in purple. Extensions to the canonical $\mathrm{PH}$ domain fold in PLC $\beta 2$, PLC $\gamma 2$, Sec3p, Grp1, ARHGAP21 and RanBP2 are shown in green.

Figure 5.2. Comparison of the PH domain surfaces that contact small G proteins.

The seven PH domains were overlaid. The Exo84 PH domain is shown in green in the centre, chosen because it does not contain any extra structural features. The $\mathrm{G}$ proteins contact completely different surfaces on the PH domain fold. They are coloured as follows: RalA (Exo84 complex) yellow; Rac1 (PLC $\beta 2$ complex) cyan; Rac2 (PLC $\gamma 2$ complex) blue; Rho1p (Sec3p complex) orange; Arf1 (ARHGAP21 complex) magenta; Arf6 (Grp1 complex) pale pink; Ran (RanBP1 complex) red. 
Figure 6.1.Sequence alignment of the small $\mathrm{G}$ proteins whose complexes have been described.

The secondary structure and numbering for Ras are shown at the top. The switch regions for Ras are also marked. The small $\mathrm{G}$ protein sequences are coloured according to the number of times each residue is involved in effector interactions for the complexes solved for that particular G protein. The colours are as follows: residues contacting all effectors, red; residues contacting all but 1, orange; residues contacting all but 2, yellow; residues contacting all but 3, green; residues contacting all but 4 , cyan; residues contacting all but 5 , light blue; residues contacting all but 6 , dark blue. For those $\mathrm{G}$ proteins with only one effector complex structure available the interacting residues are coloured orange. 

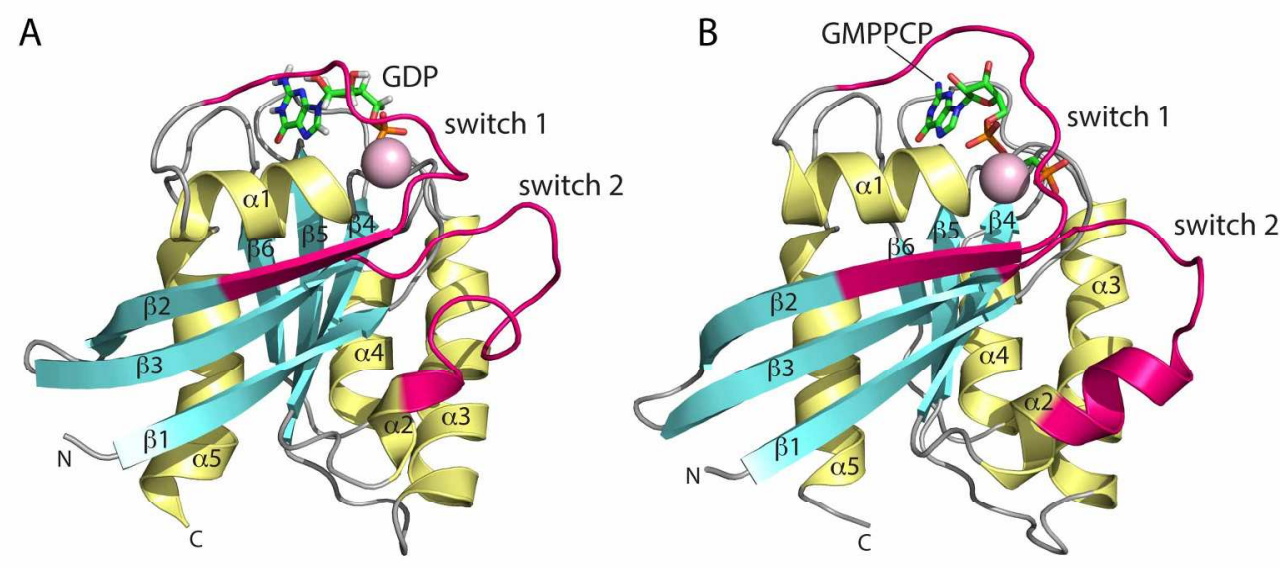

C switch 2

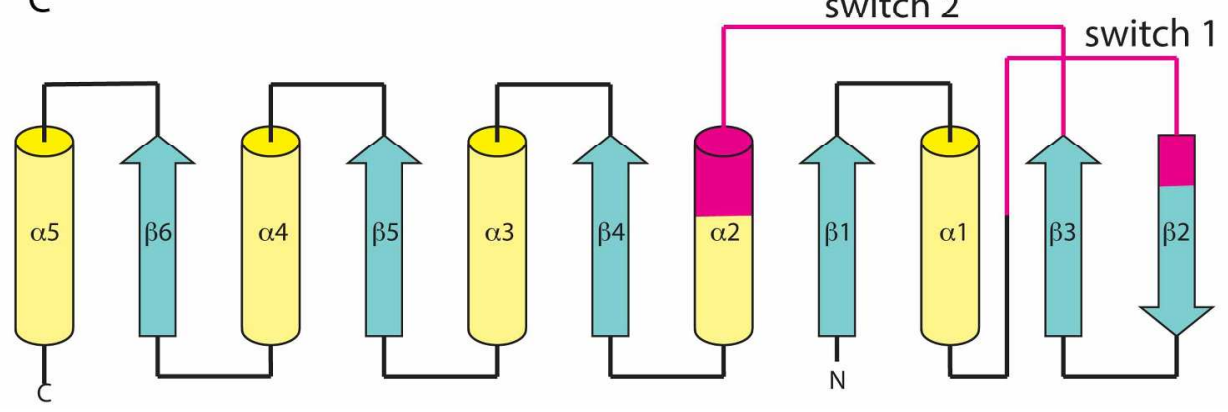

Fig 1.1

$207 \times 168 \mathrm{~mm}$ ( $300 \times 300$ DPI) 
A

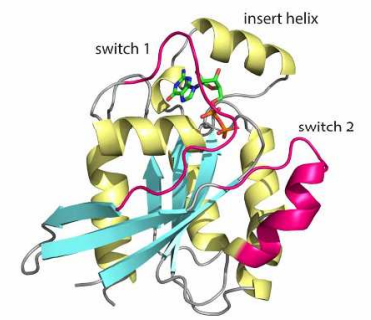

B

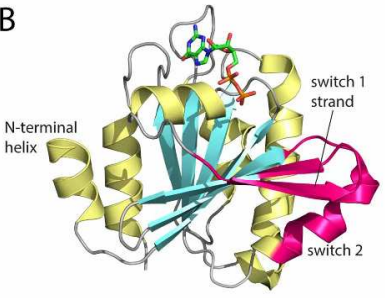

C

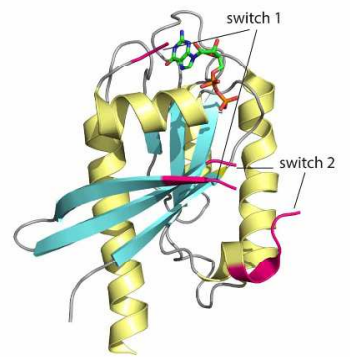

D

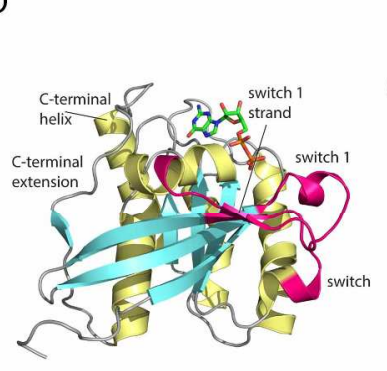

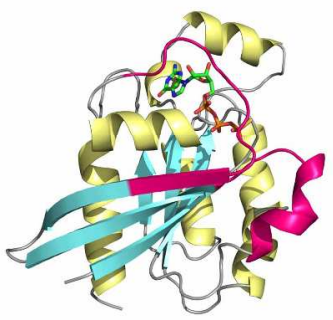
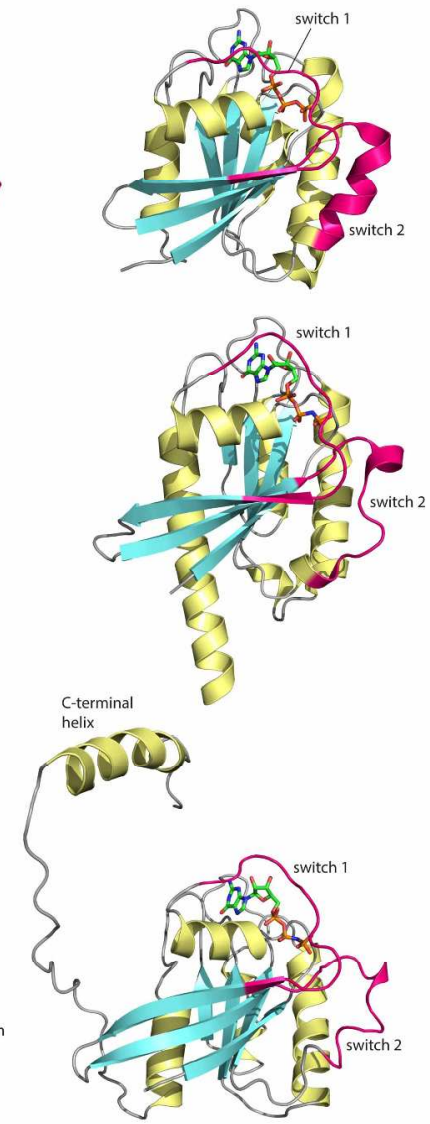

Fig 1.2

$168 \times 290 \mathrm{~mm}(300 \times 300 \mathrm{DPI})$ 
A

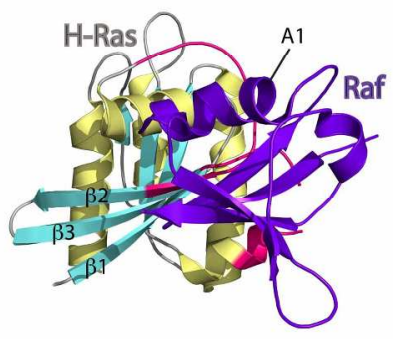

C
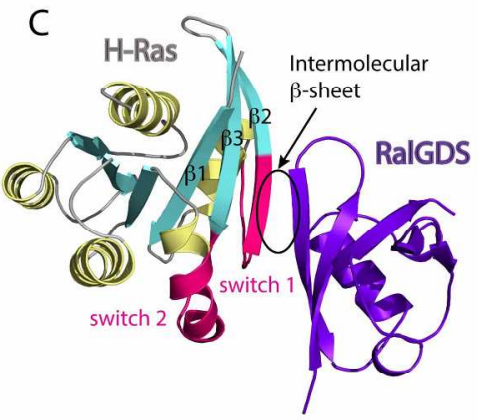

E

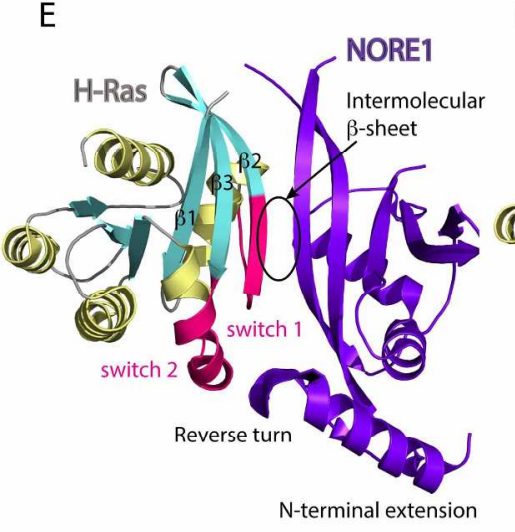

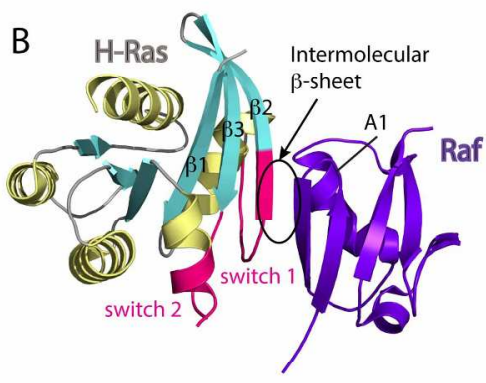

D H-Ras Intermolecular

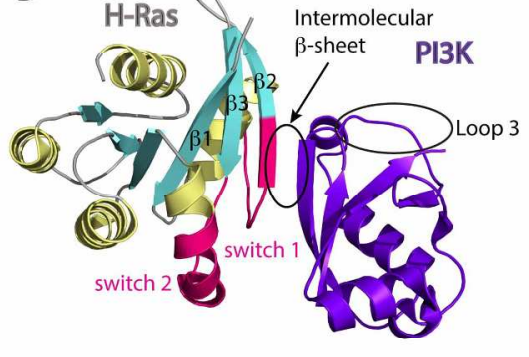

$\mathrm{F}$

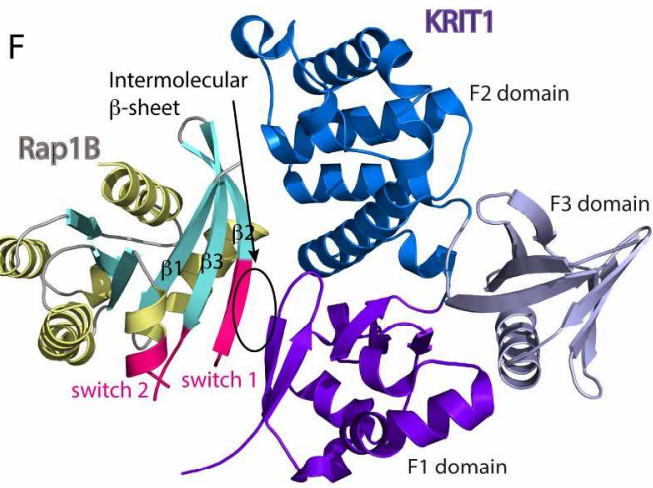

Fig 2.1

$202 \times 266 \mathrm{~mm}(300 \times 300 \mathrm{DPI})$ 
Fig 2.2

$176 \times 176 \mathrm{~mm}(300 \times 300 \mathrm{DPI})$

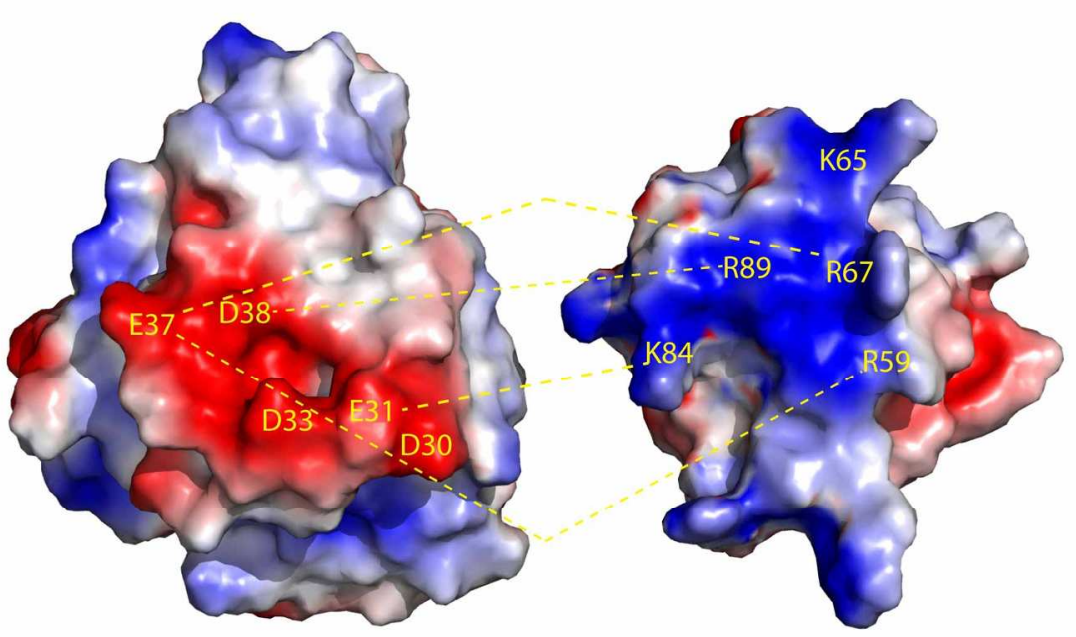



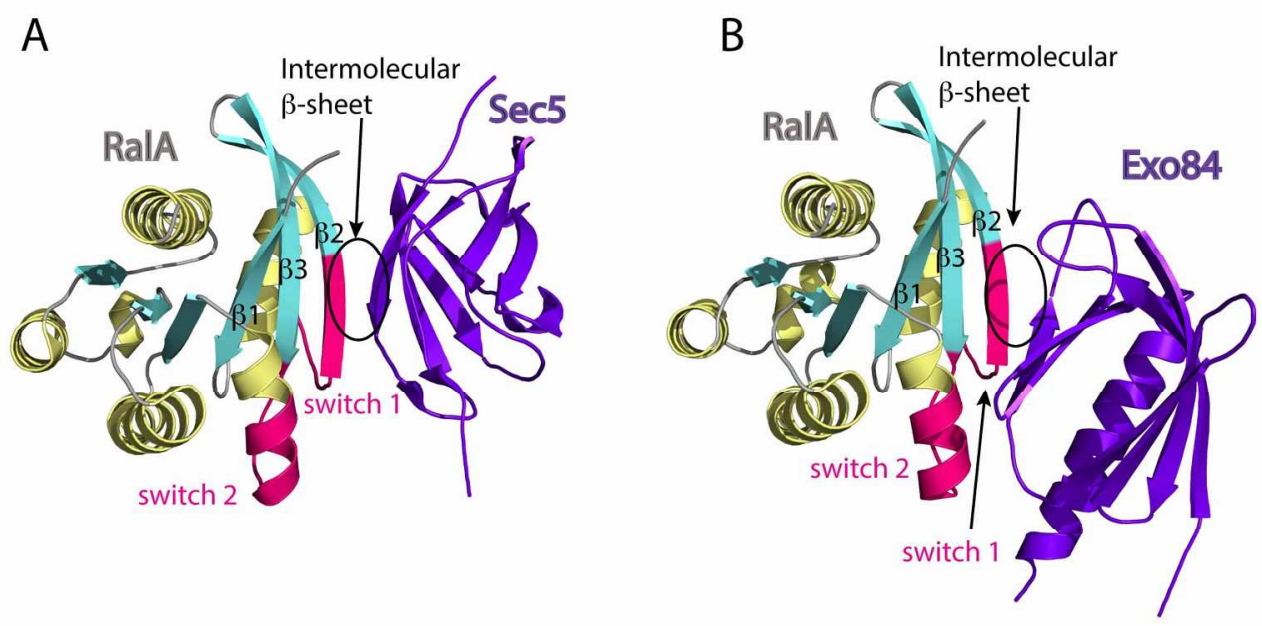

Fig 2.3

$172 \times 90 \mathrm{~mm}(300 \times 300 \mathrm{DPI})$ 

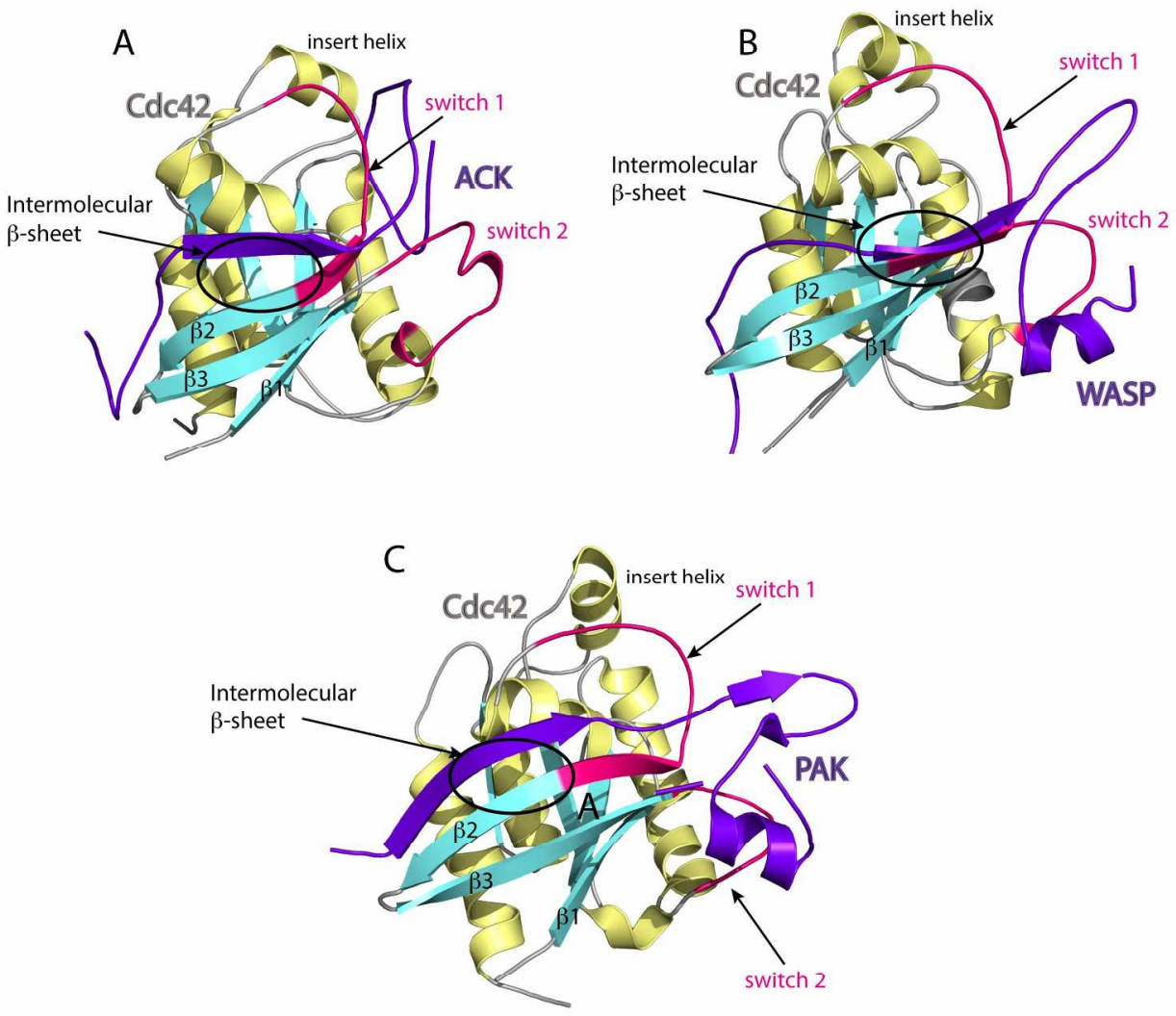

Fig 2.4

$203 \times 192 \mathrm{~mm}(300 \times 300 \mathrm{DPI})$

URL: http:/mc.manuscriptcentral.com/bbmg Email: pfeffer@biochem.wisc.edu 

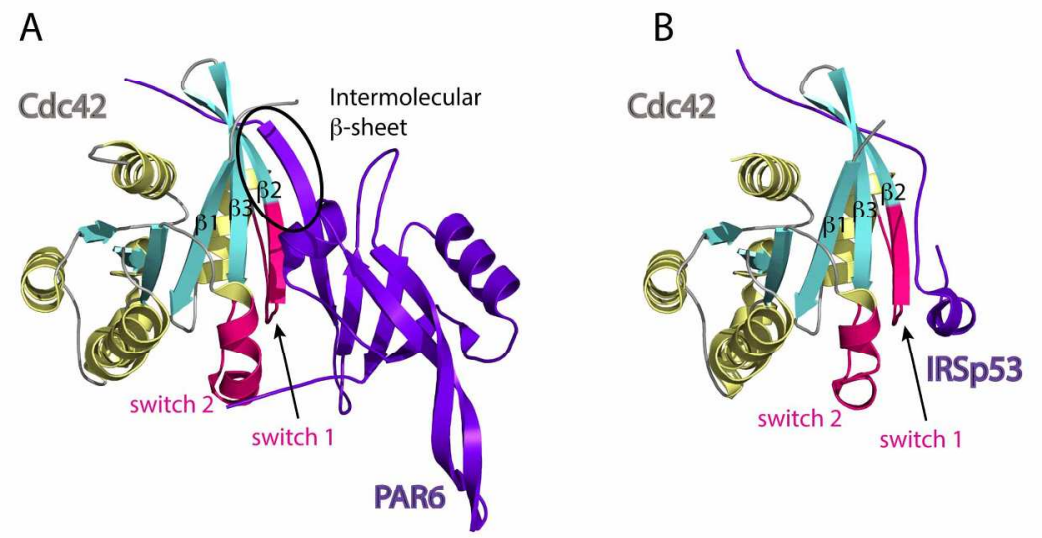

Fig 2.5

$203 \times 106 \mathrm{~mm}(300 \times 300$ DPI $)$ 

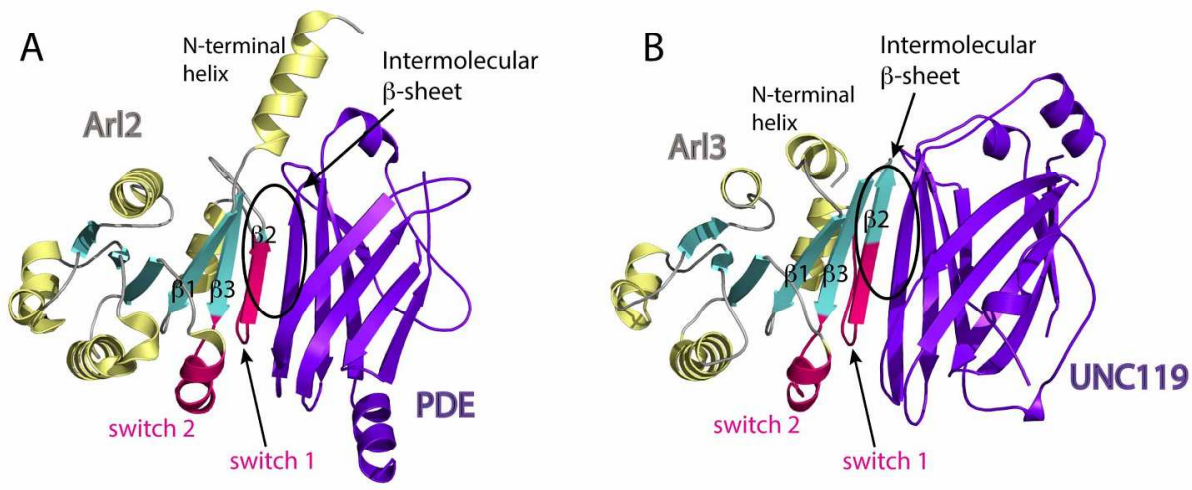

Fig 2.6

$197 \times 105 \mathrm{~mm}(300 \times 300$ DPI $)$ 
Fig 2.7

$145 \times 141 \mathrm{~mm}(300 \times 300 \mathrm{DPI})$ 


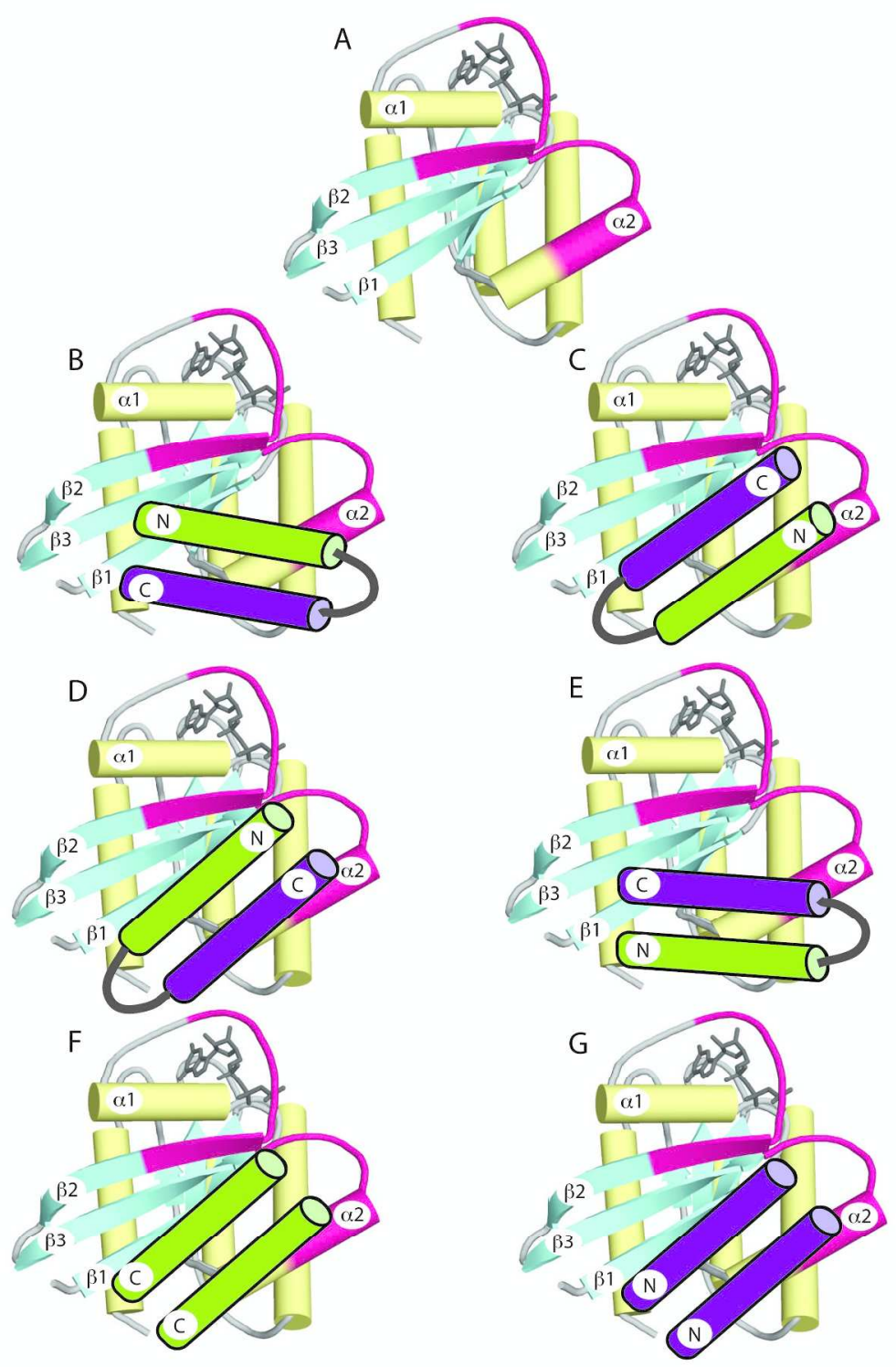

Fig 3.1

$191 \times 290 \mathrm{~mm}(300 \times 300 \mathrm{DPI})$

URL: http:/mc.manuscriptcentral.com/bbmg Email: pfeffer@biochem.wisc.edu 


1
2
3
4
5
6
7
8
9
10
11
12
13
14
15
16
17
18
19
20
21
22
23
24
25
26
27
28
29
30
31
32
33
34
35
36
37
38
39
40
41
42
43
44
45
46
47
48
49
50
51
52
53
54
55
56
50

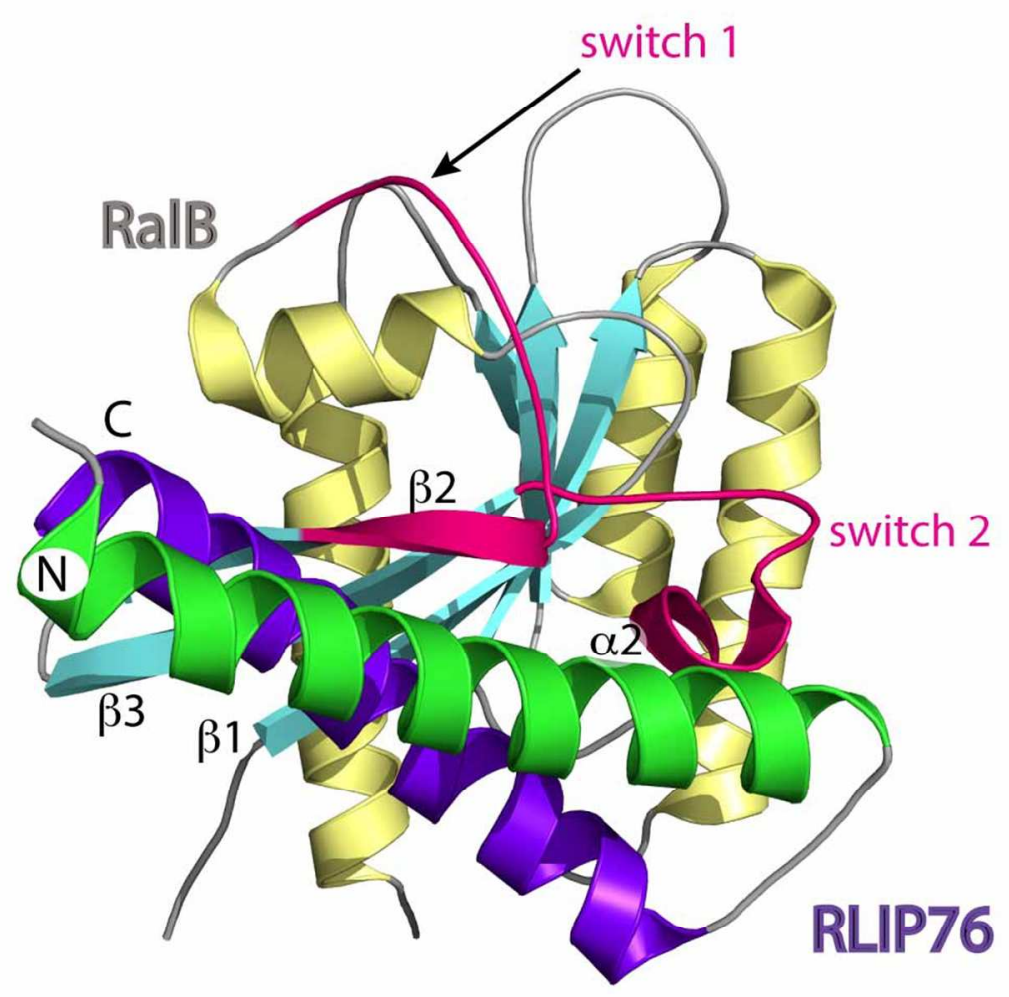

Fig 3.2

$105 \times 121 \mathrm{~mm}(300 \times 300 \mathrm{DPI})$ 

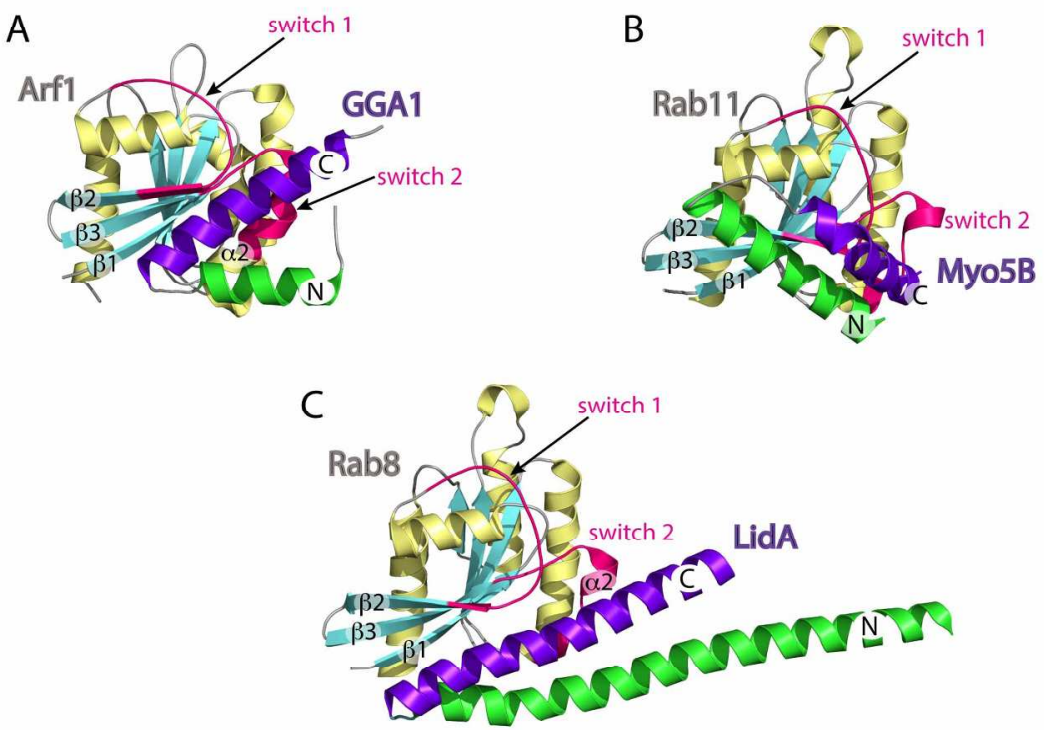

Fig 3.3

$200 \times 197 \mathrm{~mm}(300 \times 300$ DPI $)$

URL: http:/mc.manuscriptcentral.com/bbmg Email: pfeffer@biochem.wisc.edu 


1
2
3
4
5
6
7
8
9
10
11
12
13
14
15
16
17
18
19
20
21
22
23
24
25
26
27
28
29
30
31
32
33
34
35
36
37
38
39
40
59
50
50
49
50
51
52
53
44
54

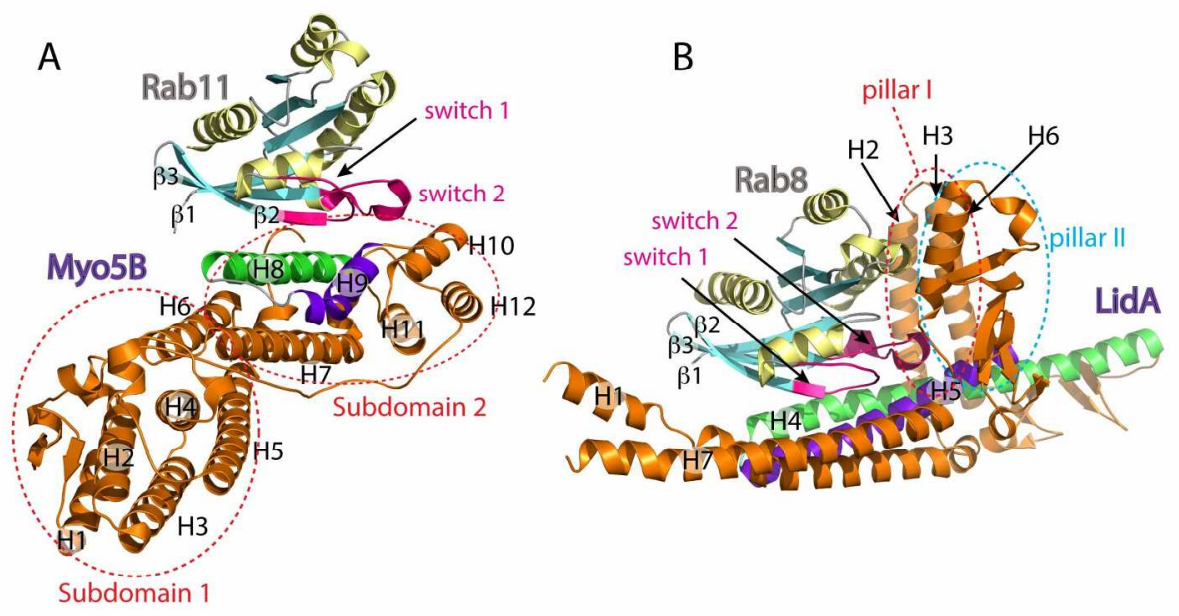

Fig 3.4

$198 \times 115 \mathrm{~mm}(300 \times 300$ DPI $)$

URL: http:/mc.manuscriptcentral.com/bbmg Email: pfeffer@biochem.wisc.edu 

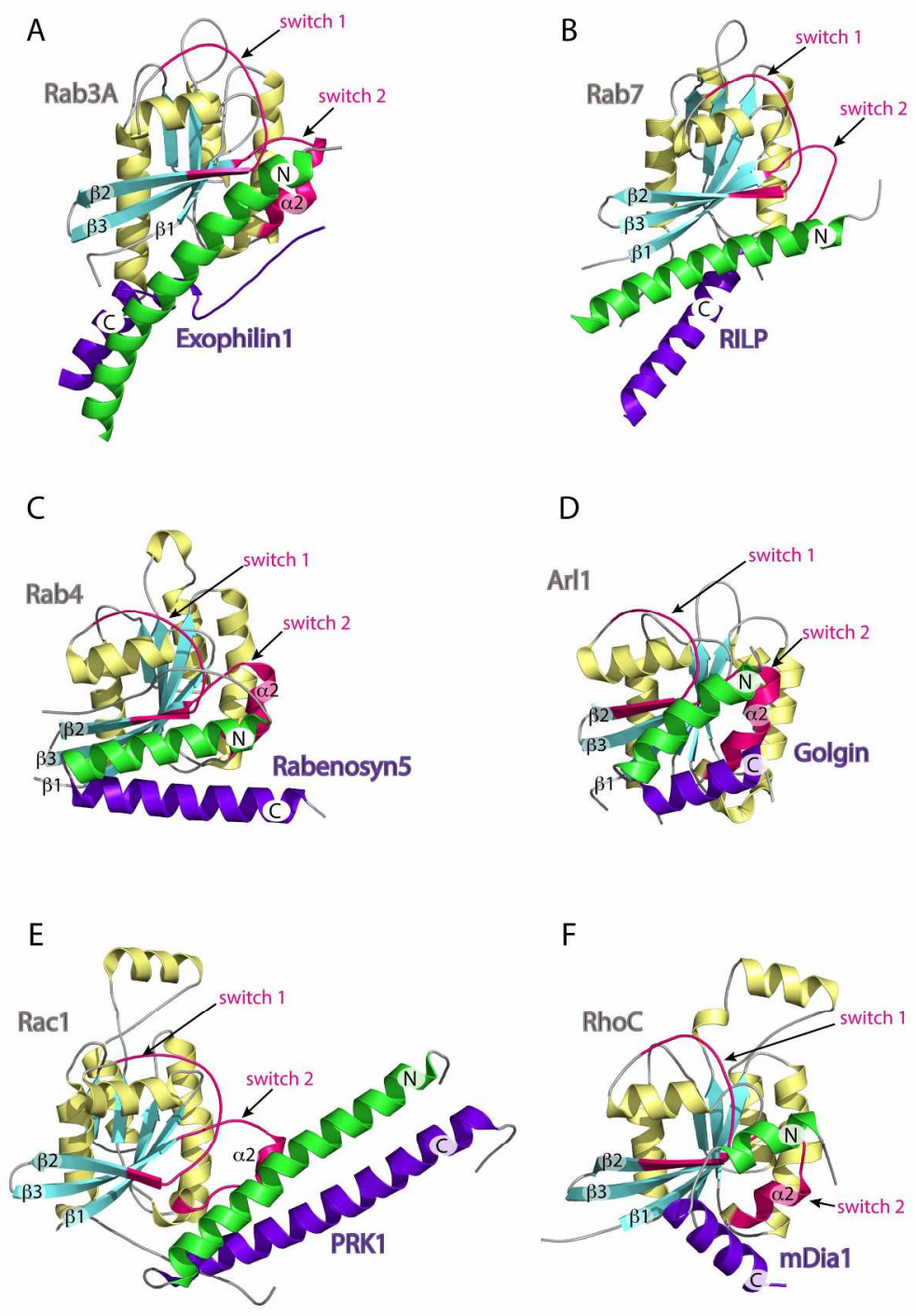

Fig 3.5

$215 \times 283 \mathrm{~mm}(300 \times 300 \mathrm{DPI})$ 

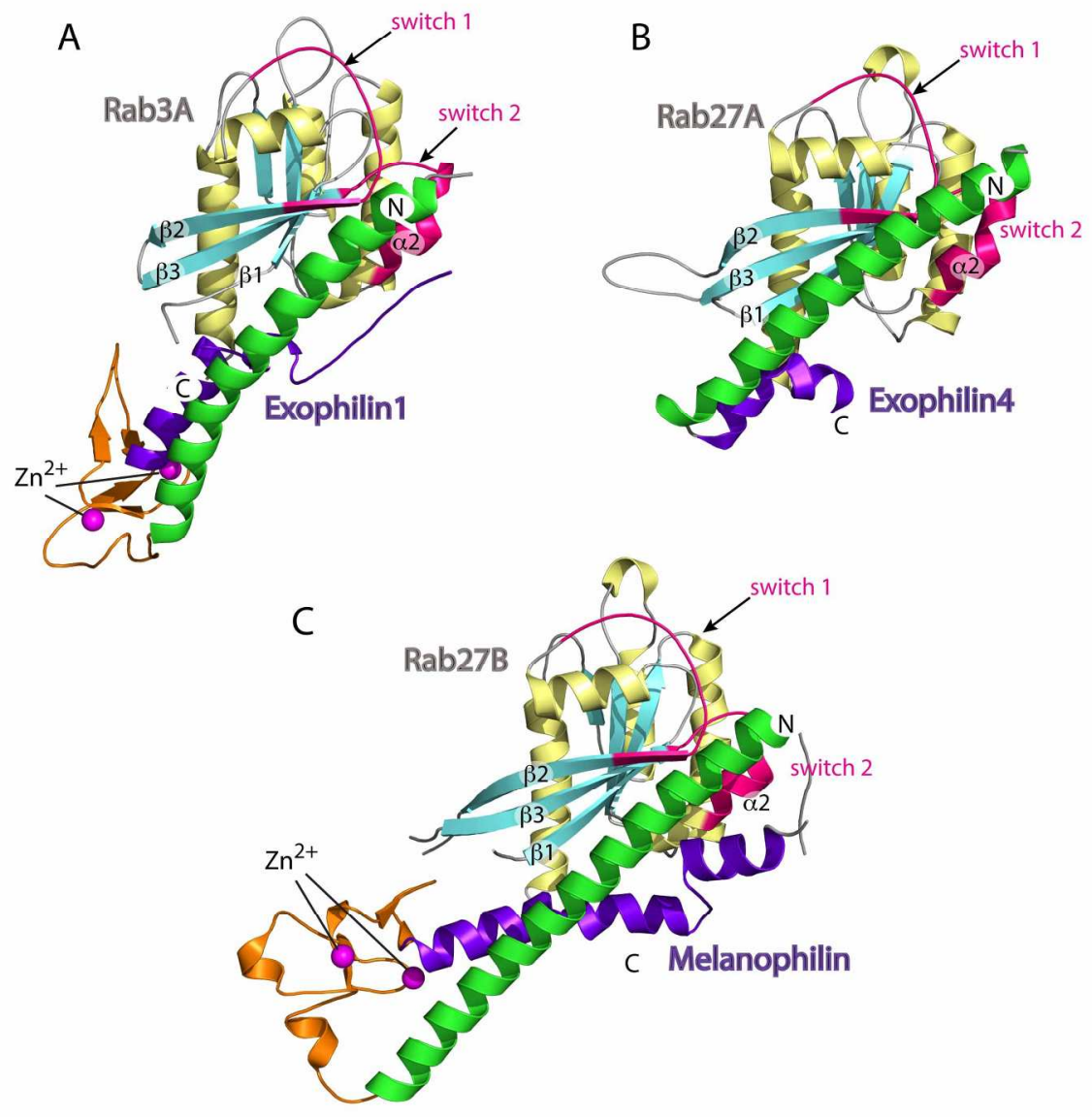

Fig 3.6

$201 \times 199 m m(300 \times 300$ DPI $)$ 


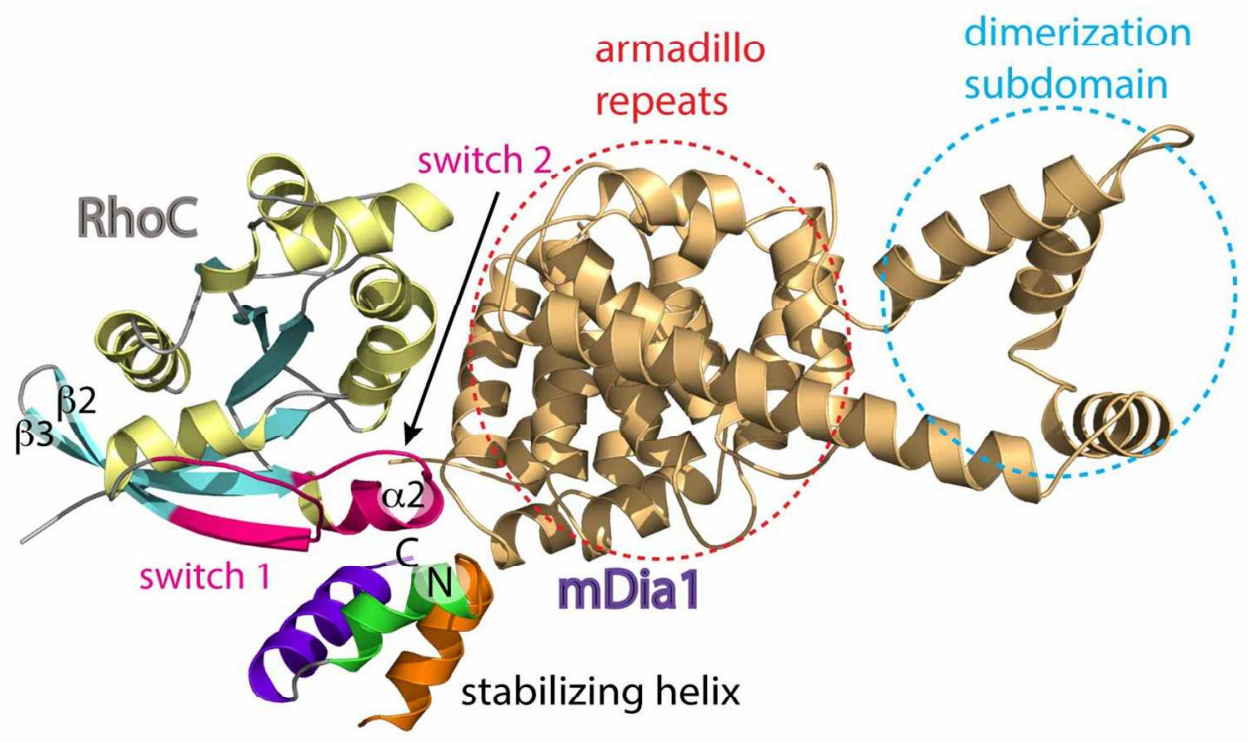

Fig 3.7

$127 \times 127 \mathrm{~mm}(300 \times 300 \mathrm{DPI})$

URL: http:/mc.manuscriptcentral.com/bbmg Email: pfeffer@biochem.wisc.edu 

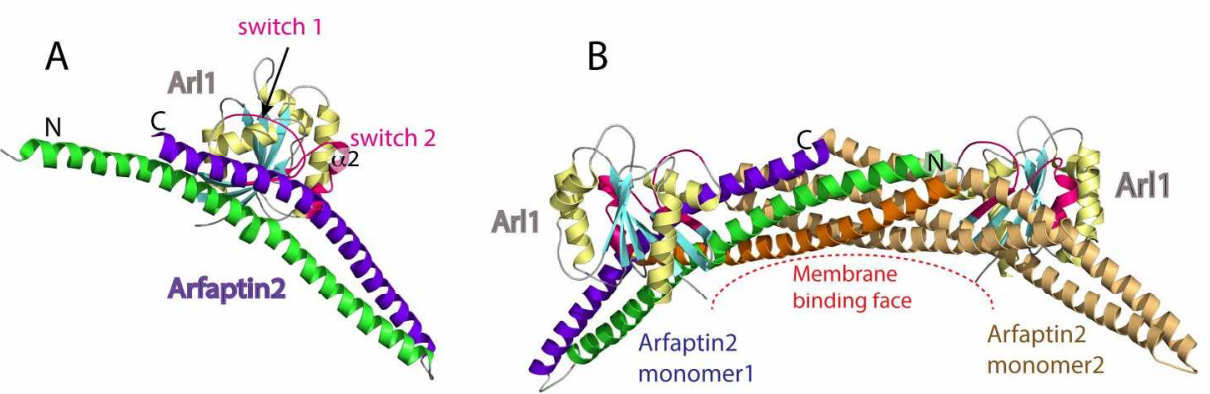

\section{C}

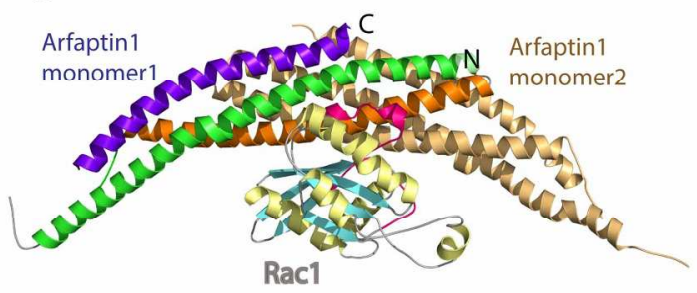

Fig 3.8

$198 \times 168 \mathrm{~mm}(300 \times 300 \mathrm{DPI})$

URL: http:/mc.manuscriptcentral.com/bbmg Email: pfeffer@biochem.wisc.edu 

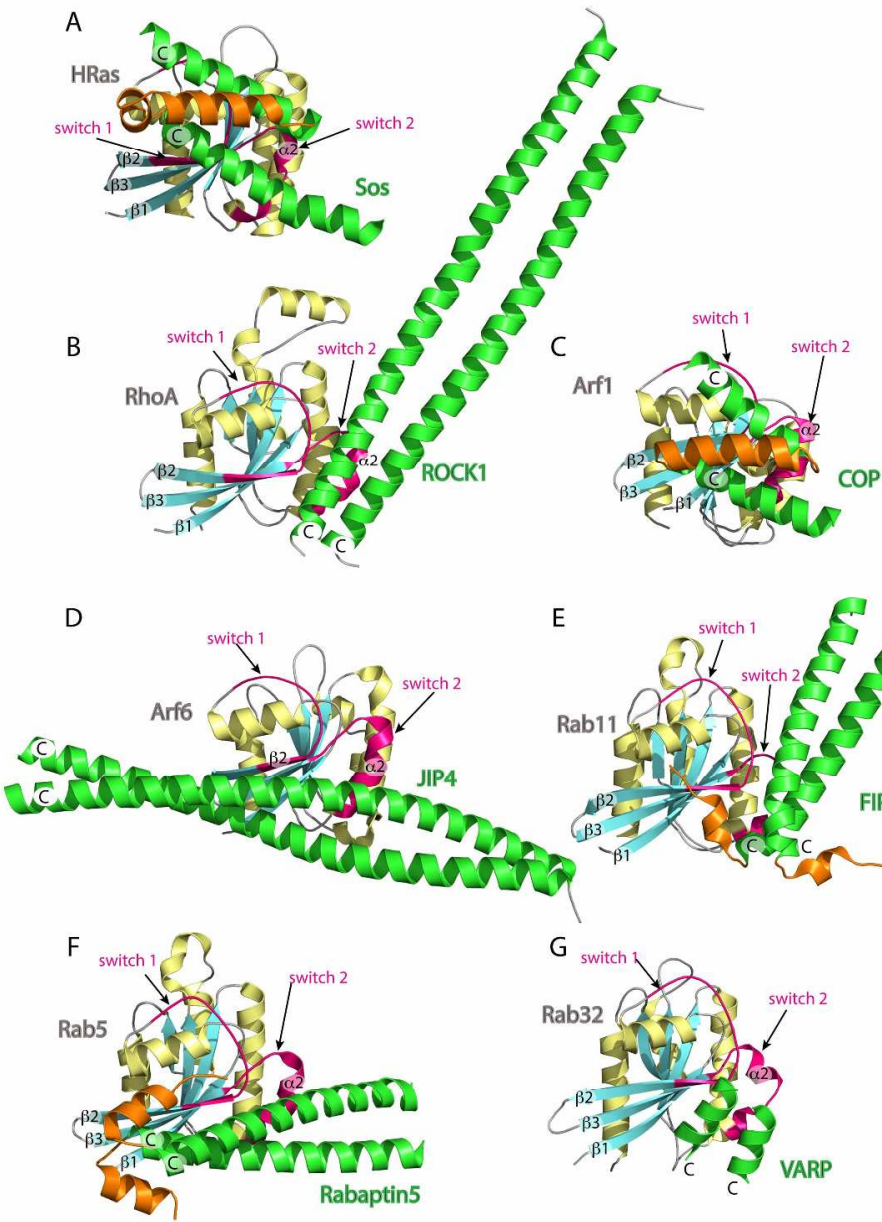

E
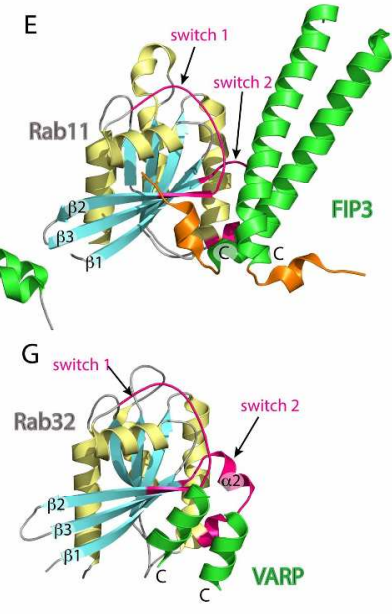

Fig 3.9

$253 \times 350 \mathrm{~mm}(300 \times 300 \mathrm{DPI})$

URL: http:/mc.manuscriptcentral.com/bbmg Email: pfeffer@biochem.wisc.edu 


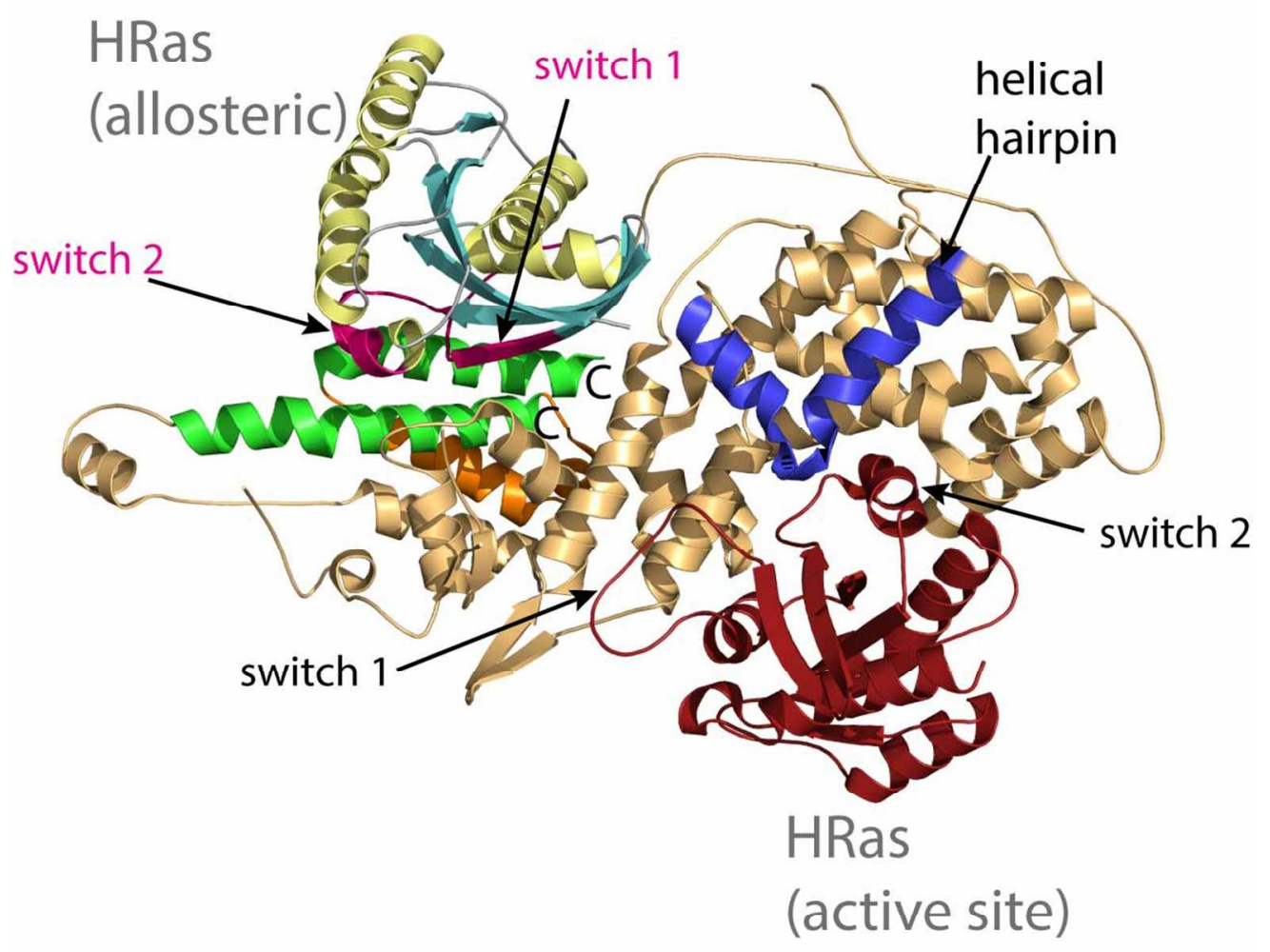

Fig 3.10

$107 \times 105 \mathrm{~mm}(300 \times 300$ DPI $)$

URL: http:/mc.manuscriptcentral.com/bbmg Email: pfeffer@biochem.wisc.edu 

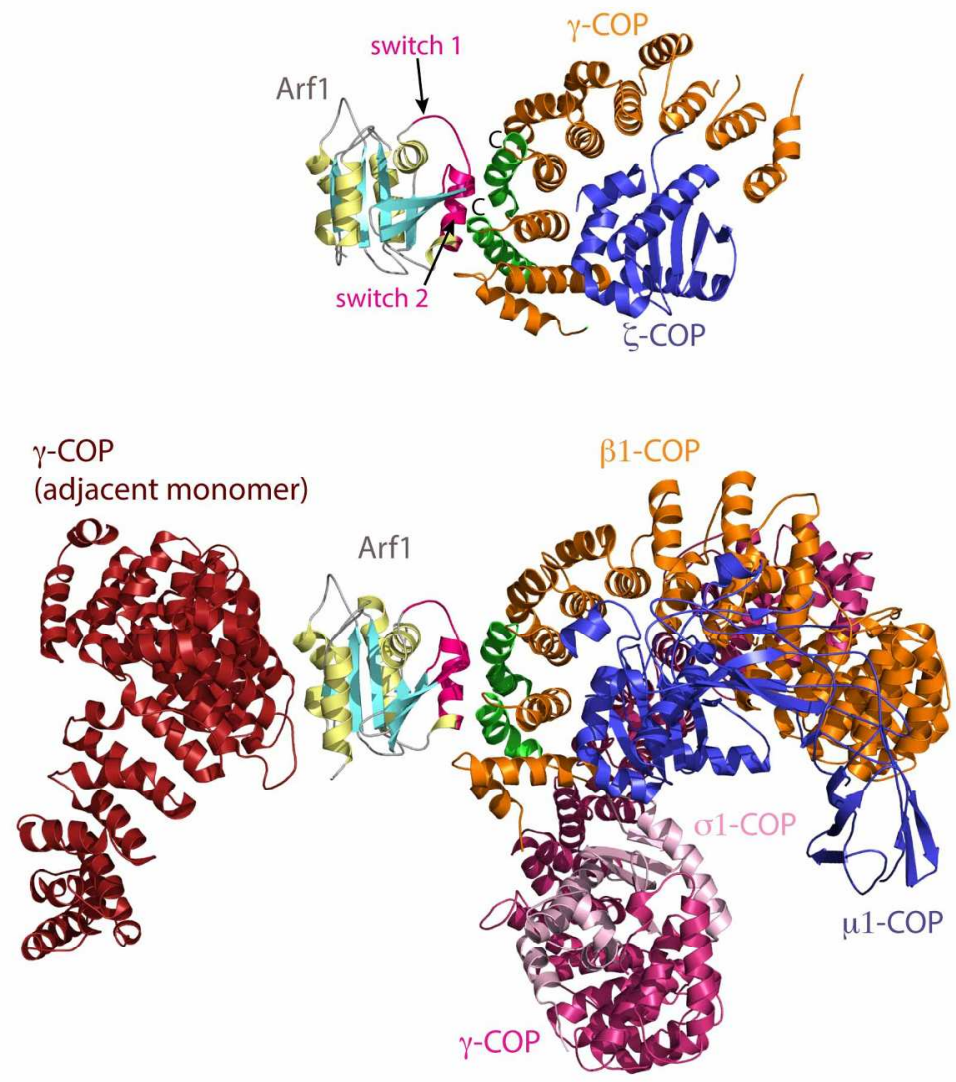

Fig 3.11

$162 \times 221 \mathrm{~mm}(300 \times 300$ DPI $)$ 


1
2
3
4
5
6
7
8
9
10
11
12
13
14
15
16
17
18
19
20
21
22
23
24
25
26
27
28
29
30
31
32
33
34
35
36
37
38
39
40
41
42
43
44
45
46
47
48
49
50
51
52
53
54
55
56
57
58
60

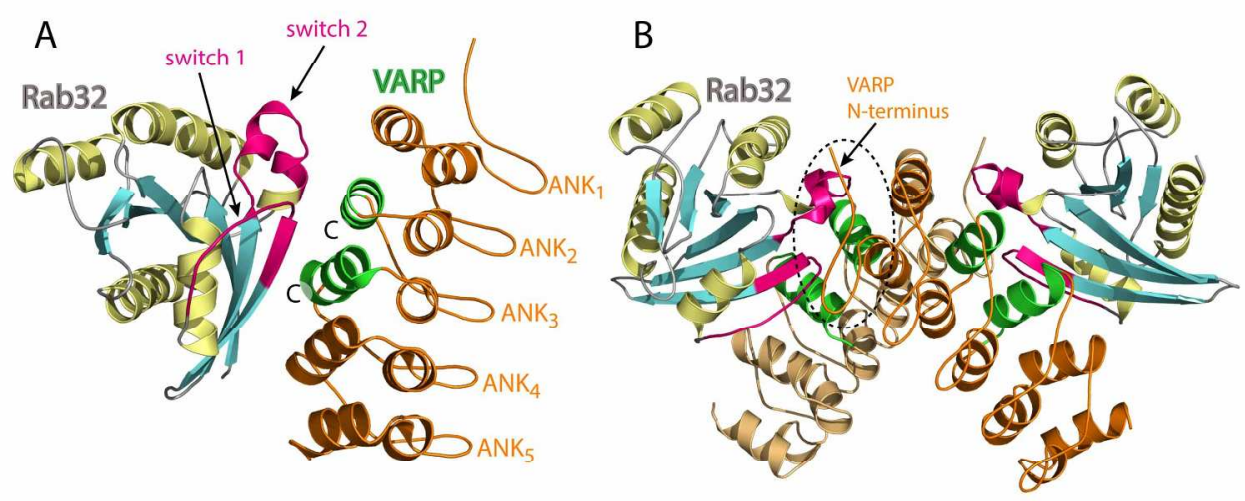

Fig 3.12

$210 \times 107 \mathrm{~mm}$ (300 x 300 DPI)

URL: http:/mc.manuscriptcentral.com/bbmg Email: pfeffer@biochem.wisc.edu 

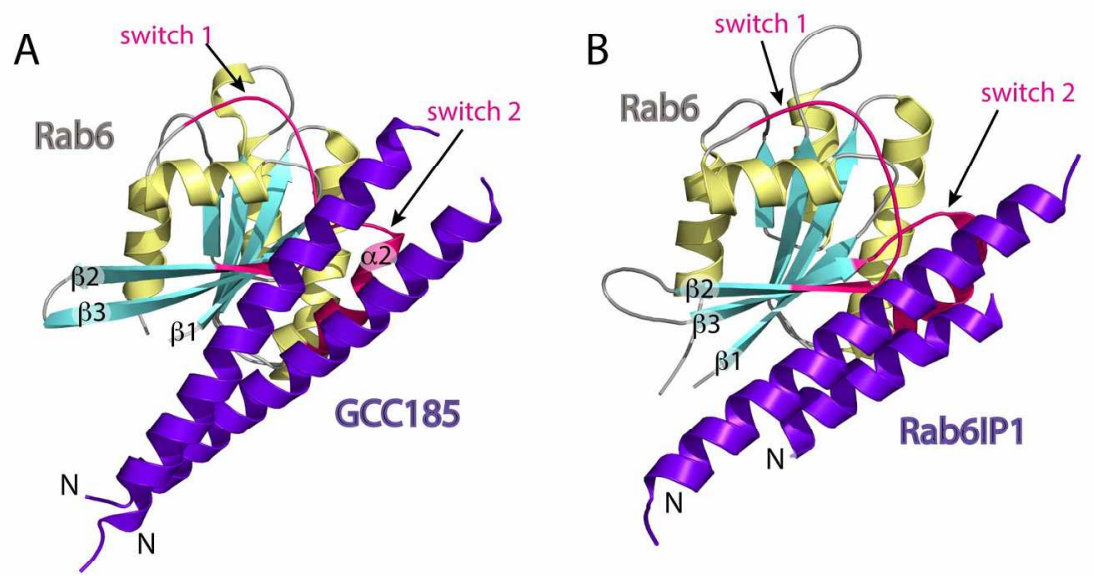

Fig 3.13

$181 \times 110 \mathrm{~mm}(300 \times 300$ DPI $)$ 


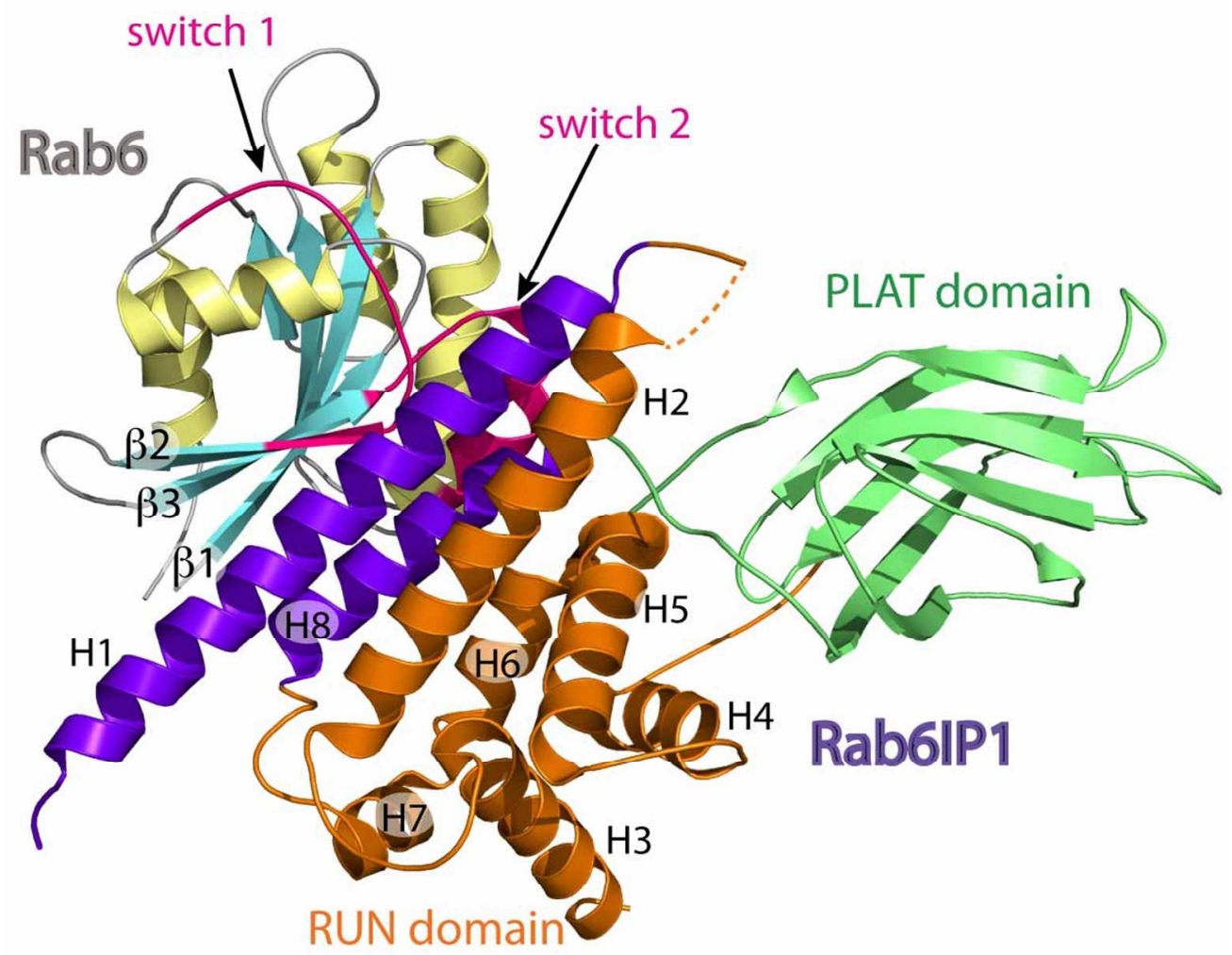

Fig 3.14

$105 \times 105 \mathrm{~mm}(300 \times 300$ DPI $)$

URL: http:/mc.manuscriptcentral.com/bbmg Email: pfeffer@biochem.wisc.edu 


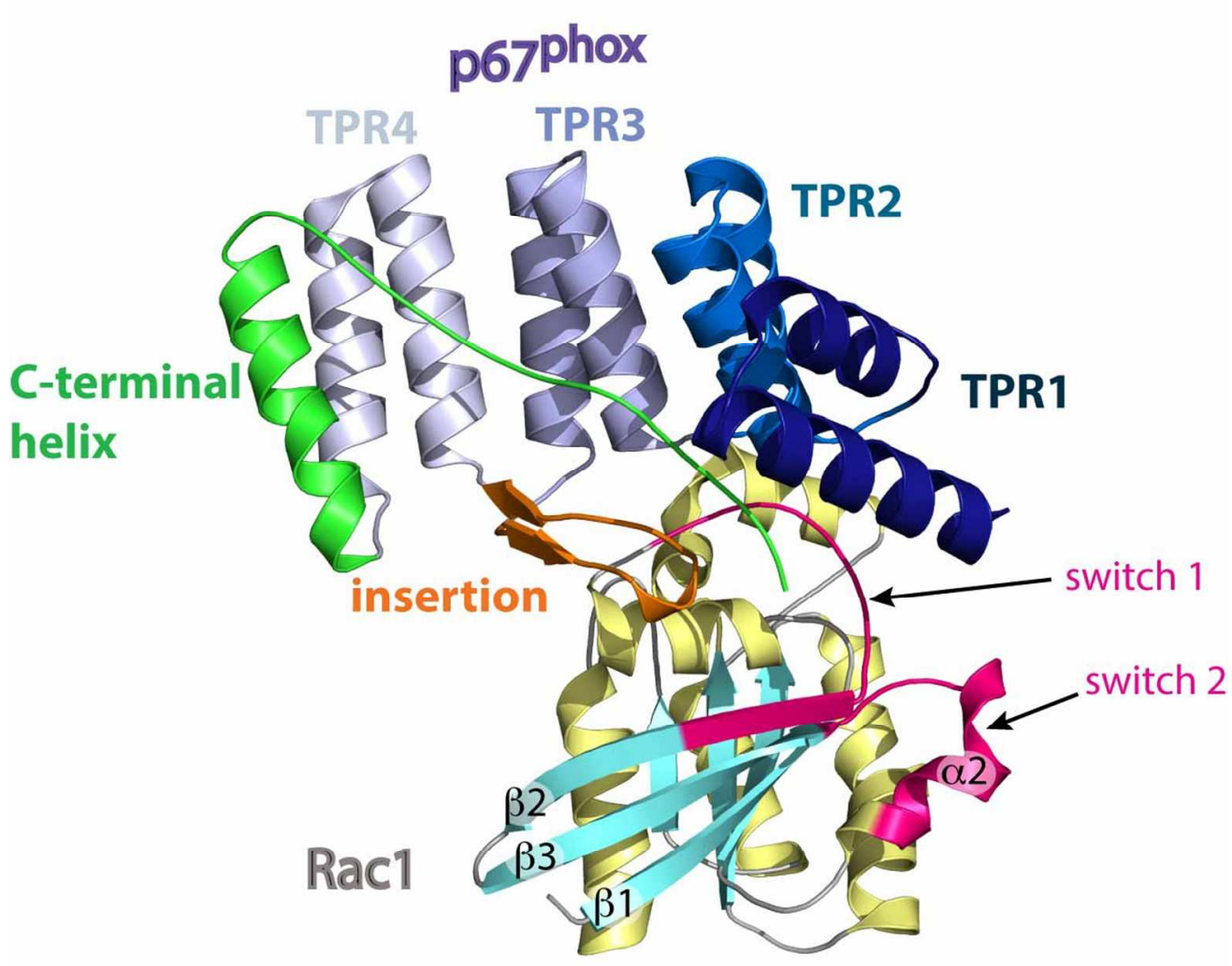

Fig 4.1

$112 \times 105 \mathrm{~mm}(300 \times 300 \mathrm{DPI})$

40

41

42

43

44

45

46

47

48

49

50

51

52

53

54

55

56

57

58

59

60

URL: http:/mc.manuscriptcentral.com/bbmg Email: pfeffer@biochem.wisc.edu 


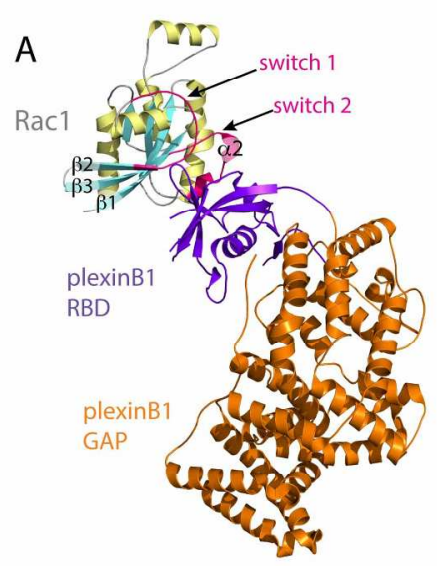

B

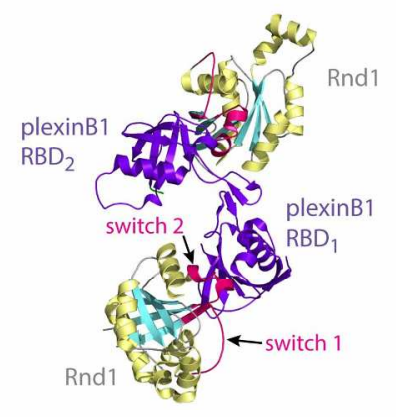

C
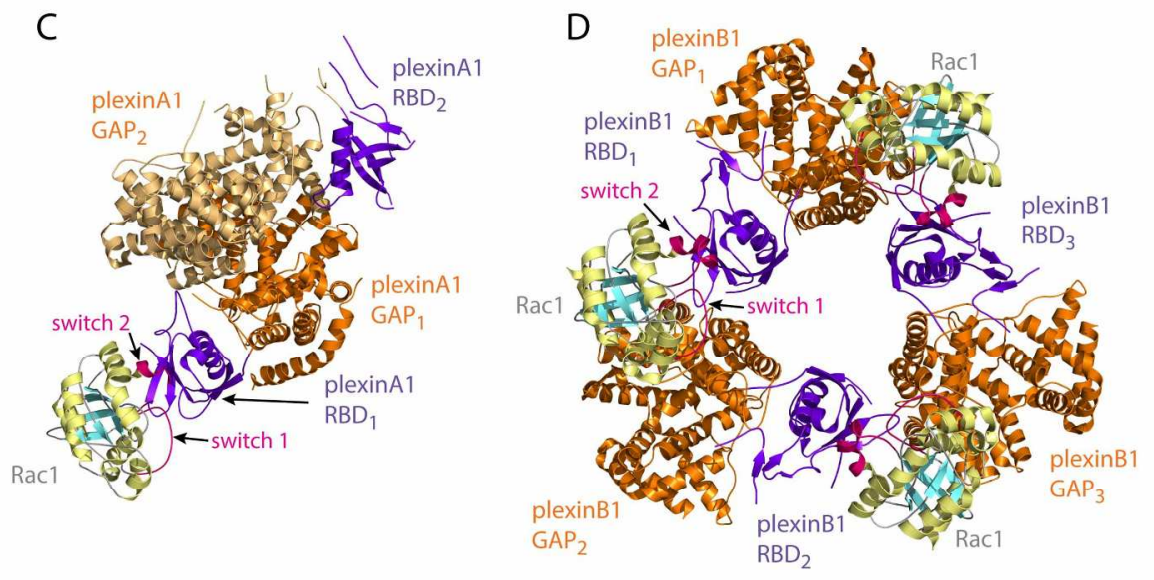

Fig 4.2

$218 \times 221 \mathrm{~mm}(300 \times 300 \mathrm{DPI})$

URL: http:/mc.manuscriptcentral.com/bbmg Email: pfeffer@biochem.wisc.edu 

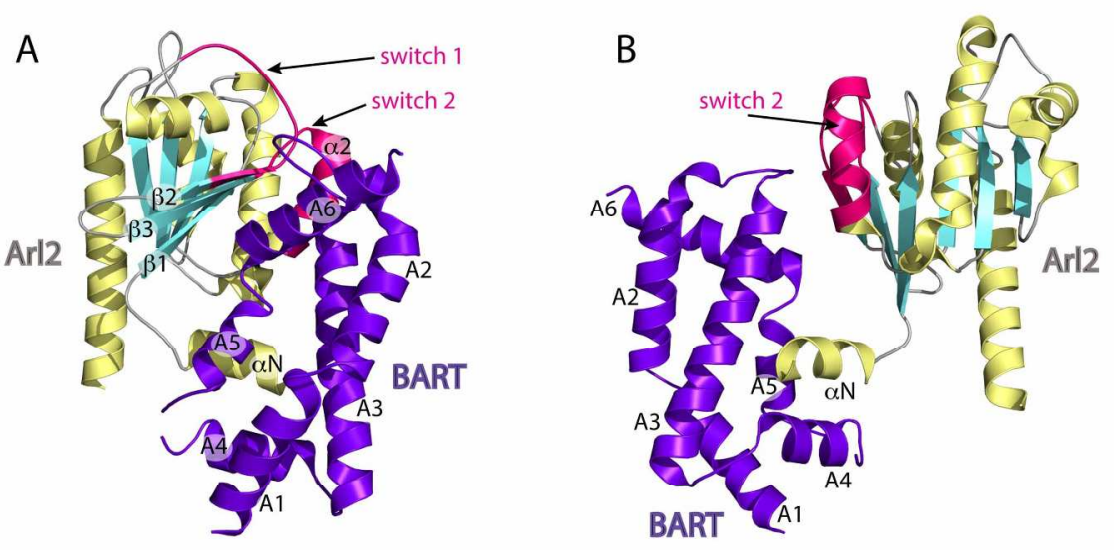

Fig 4.3

$204 \times 107 \mathrm{~mm}(300 \times 300$ DPI $)$ 


1
2
3
4
5
6
7
8
9
10
11
12
13
14
15
16
17
18
19
20
21
22
23
24
25
26
27
28
29
30
31
32
33
34
35
36
37
38
39
40
41
42
43
44
45
46
47
48
49
50
51
52
53
54
55
56
57
58
60
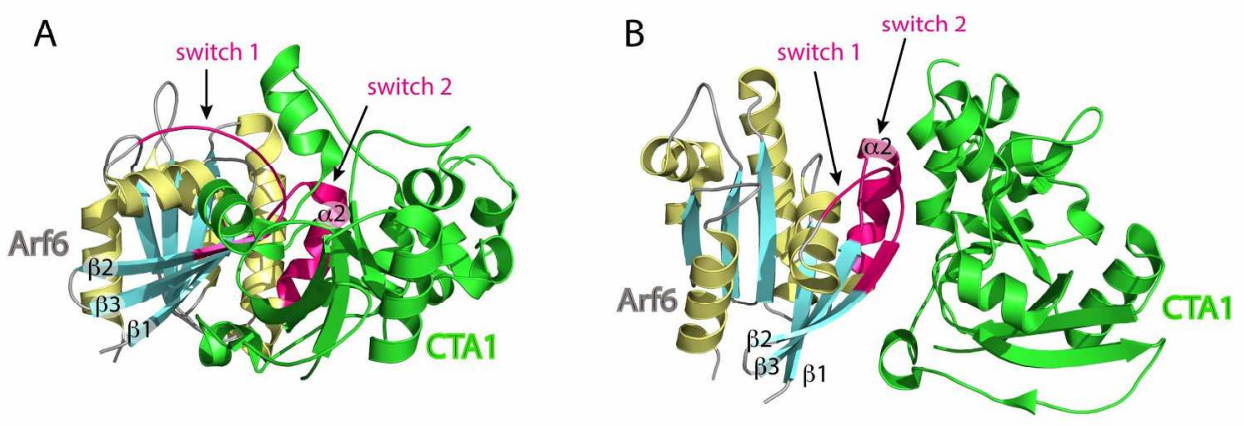

Fig 4.4

$201 \times 106 \mathrm{~mm}(300 \times 300$ DPI $)$

URL: http:/mc.manuscriptcentral.com/bbmg Email: pfeffer@biochem.wisc.edu 


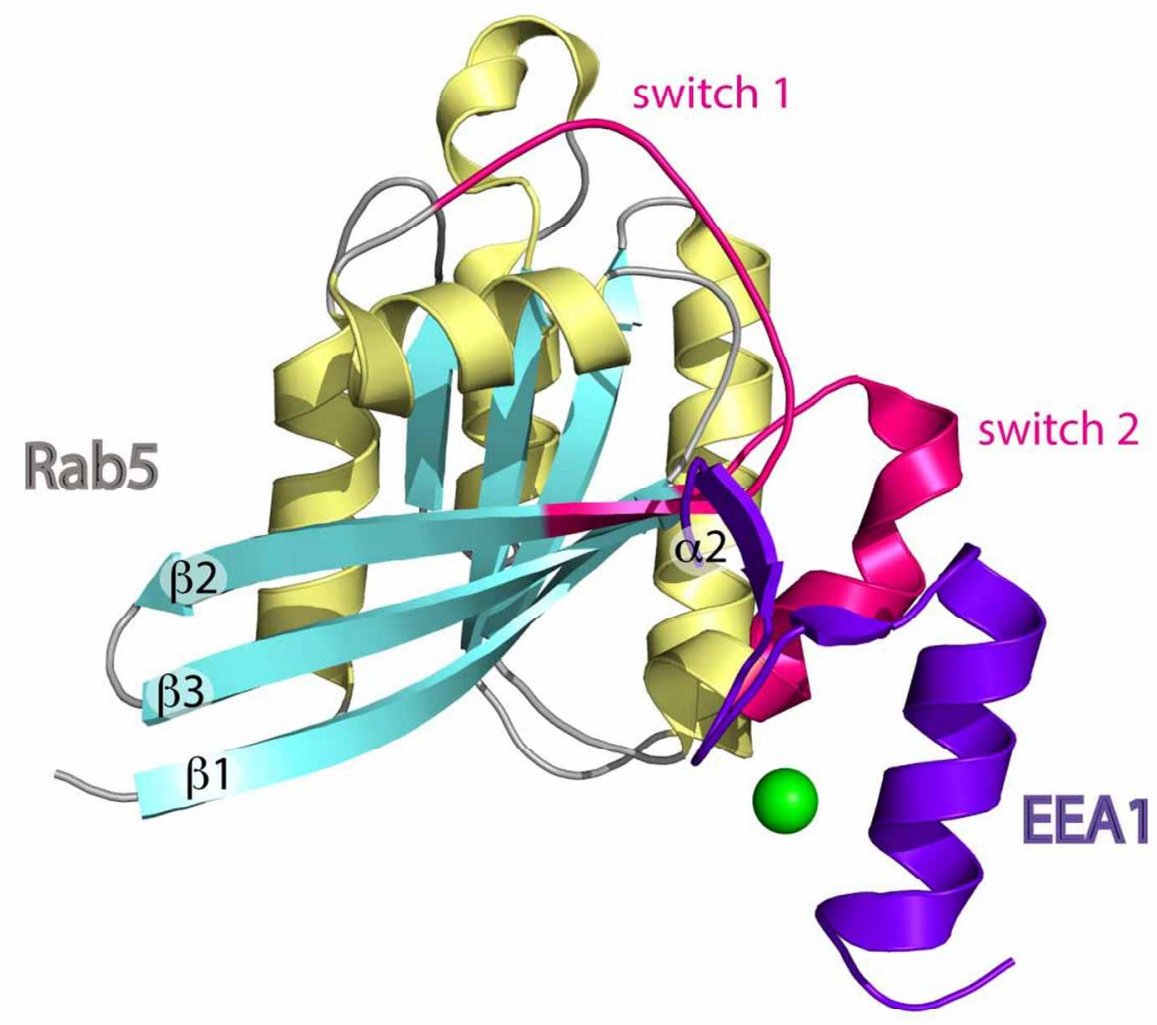

Fig 4.5

$105 \times 105 \mathrm{~mm}(300 \times 300 \mathrm{DPI})$ 

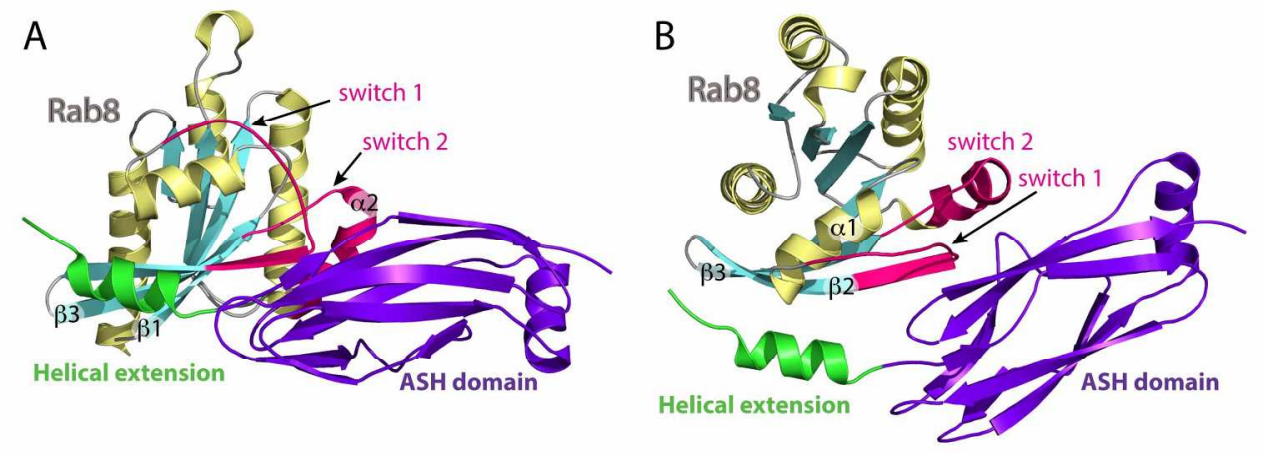

Fig 4.6

$199 \times 109 \mathrm{~mm}(300 \times 300$ DPI $)$ 

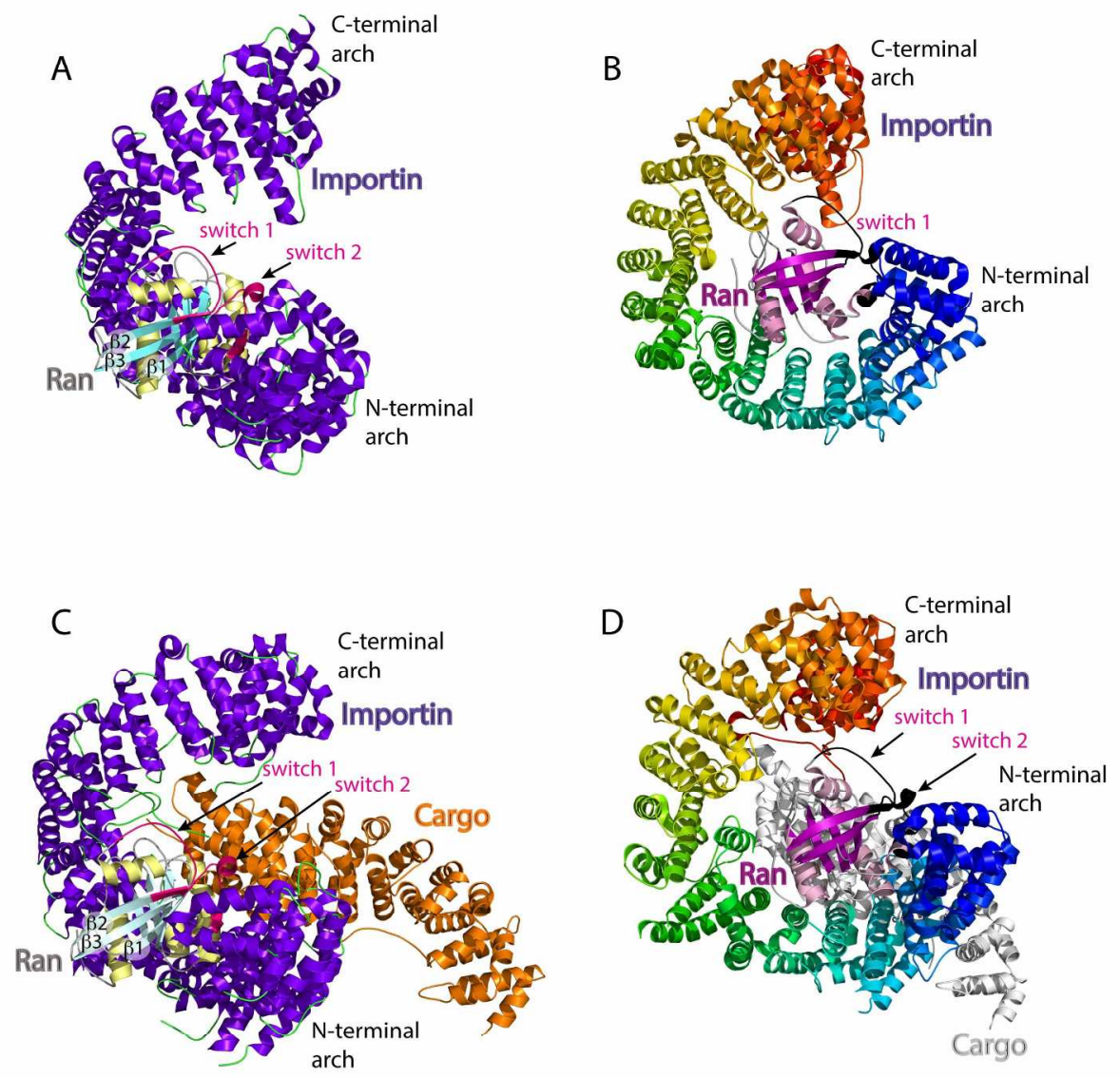

Fig 4.7

$214 \times 231 \mathrm{~mm}(300 \times 300 \mathrm{DPI})$

URL: http:/mc.manuscriptcentral.com/bbmg Email: pfeffer@biochem.wisc.edu 
A

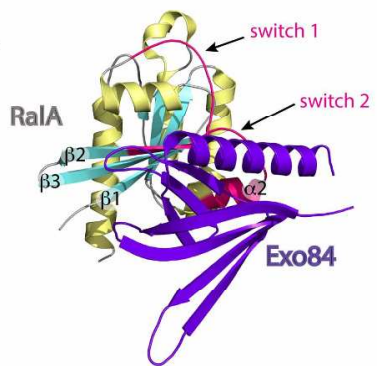

C
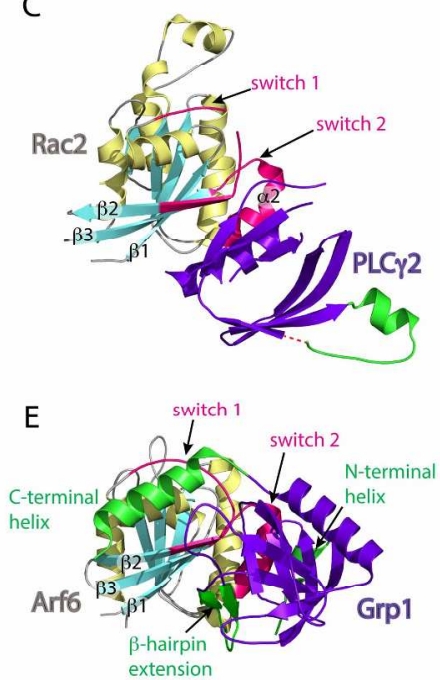

G

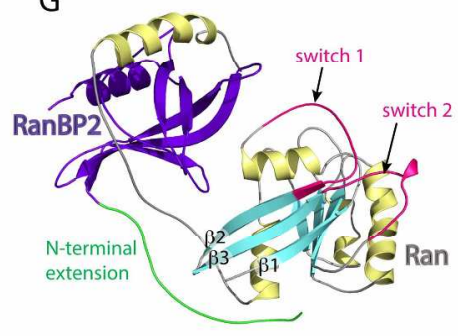

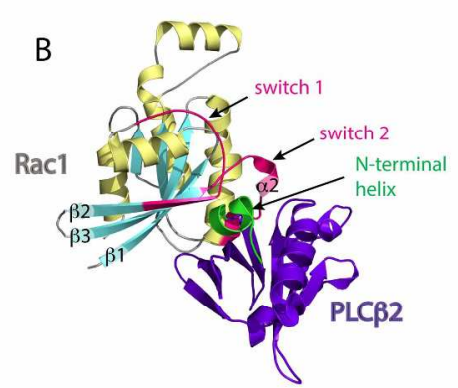

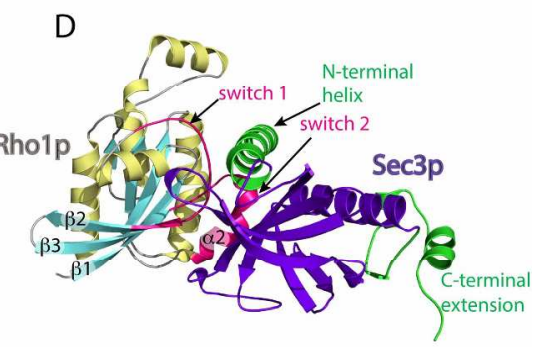

$\mathrm{F}$

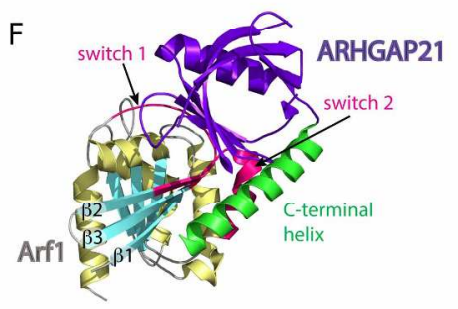

Fig 5.1

$189 \times 294 \mathrm{~mm}(300 \times 300 \mathrm{DPI})$

URL: http:/mc.manuscriptcentral.com/bbmg Email: pfeffer@biochem.wisc.edu 


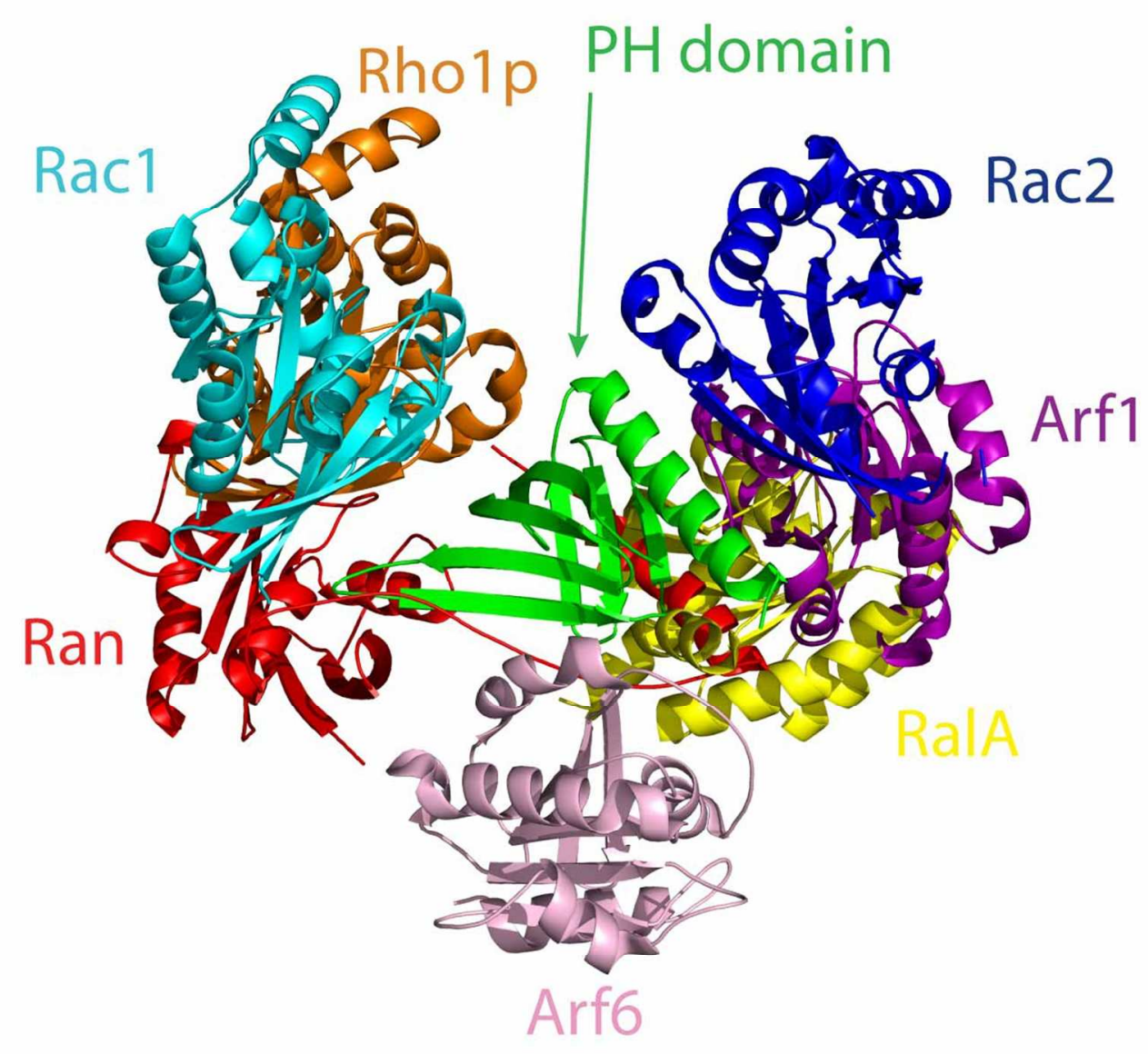

Fig 5.2

$111 \times 105 \mathrm{~mm}(300 \times 300$ DPI $)$ 


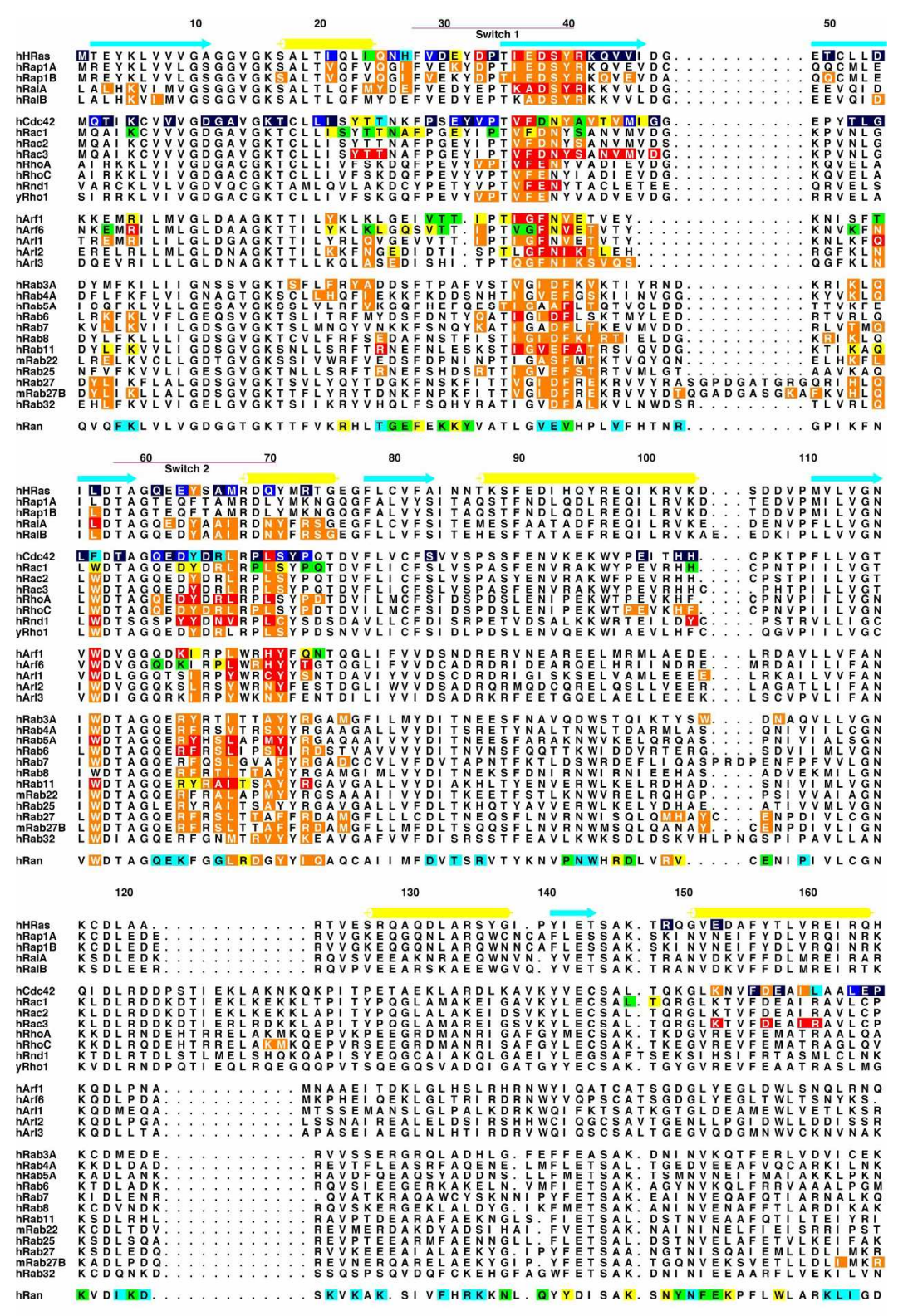

Fig 6.1

$160 \times 238 \mathrm{~mm}(300 \times 300 \mathrm{DPI})$

URL: http:/mc.manuscriptcentral.com/bbmg Email: pfeffer@biochem.wisc.edu 
Table 1 Summary of Ras family G protein-effector complex structures.

\begin{tabular}{|c|c|c|c|c|}
\hline GTPase & Effector & Structural class & PDB & Interface area $\left(\AA^{2}\right)$ \\
\hline \multicolumn{5}{|c|}{ Ras family } \\
\hline \multirow[t]{8}{*}{ H-Ras } & Raf & Intermolecular- $\beta$ & $4 \mathrm{GON}$ & 1228 \\
\hline & PI3 kinase & Intermolecular $-\beta$ & 1HE8 & 1305 \\
\hline & Byr2 & Intermolecular $-\beta$ & $1 \mathrm{~K} 8 \mathrm{R}$ & 1082 \\
\hline & RalGDS & Intermolecular $-\beta$ & 1LFD & 1164 \\
\hline & PLC e & Intermolecular $-\beta$ & $2 \mathrm{C} 5 \mathrm{~L}$ & 1180 \\
\hline & Nore1 & Intermolecular $-\beta$ & $3 \mathrm{DDC}$ & 1576 \\
\hline & Grb14 & Intermolecular $-\beta$ & $4 \mathrm{~K} 81$ & 885 \\
\hline & Sos & Helical pair (F) & $1 \mathrm{NVV}$ & 3168 \\
\hline Rap1A & Riam & Intermolecular $-\beta$ & $4 \mathrm{KVG}$ & 1037 \\
\hline Rap1B & KRIT1 & Intermolecular $-\beta$ & 4HDO, 4DXA & 1536,1749 \\
\hline \multirow[t]{2}{*}{ RalA } & Sec5 & Intermolecular $-\beta$ & 1UAD & 1013 \\
\hline & Exo84 & Intermolecular $-\beta$ PH domain & $1 \mathrm{ZC} 3$ & 1801 \\
\hline \multirow[t]{2}{*}{ RalB } & RalBP1 & Helical pair (B) & $2 \mathrm{KWI}$ & 1673 \\
\hline & & Rho family & & \\
\hline \multirow[t]{7}{*}{ Cdc42 } & ACK & Intermolecular $-\beta$ & $1 \mathrm{CF} 4$ & 4021 \\
\hline & PAK1 & Intermolecular $-\beta$ & $1 \mathrm{E} 0 \mathrm{~A}$ & 2637 \\
\hline & PAK6 & Intermolecular $-\beta$ & 2ODB & 2027 \\
\hline & WASP & Intermolecular $-\beta$ & $1 \mathrm{CEE}$ & 2827 \\
\hline & Par6 & Intermolecular $-\beta$ & 1NF3 & 2307 \\
\hline & Irsp53 & Intermolecular $-\beta$ & $4 \mathrm{JSO}$ & 2291 \\
\hline & mDia & Helical pair (D) & 3EG5 & 2007 \\
\hline \multirow[t]{5}{*}{ Rac1 } & PRK1 & Helical pair (D) & 2RMK & 2052 \\
\hline & Phox & Other & 1E96 & 1180 \\
\hline & PLC $\beta 2$ & PH domain & $2 \mathrm{FJU}$ & 1252 \\
\hline & PlexinB1 & Other & 3SU8 3SUA & 1429 \\
\hline & Plexin A1 & Other & 3RYT & 1145 \\
\hline Rac2 & PLC $\gamma 2$ & PH domain & $2 \mathrm{~W} 2 \mathrm{X}$ & 950 \\
\hline \multirow[t]{2}{*}{ Rac3 } & PAK1 & Intermolecular $-\beta$ & 2QME & 1690 \\
\hline & PAK4 & Intermolecular $-\beta$ & $2 \mathrm{OV} 2$ & 2052 \\
\hline \multirow[t]{2}{*}{ RhoA } & PRK1 & Helical pair (D) & $1 \mathrm{CXZ}$ & 1441 \\
\hline & ROCK1 & Helical pair $(\mathrm{F})$ & $1 \mathrm{~S} 1 \mathrm{C}$ & X: 639 Y: 739 \\
\hline RhoC & $\mathrm{mDia}$ & Helical pair (D) & $1 \mathrm{Z2C}$ & 2476 \\
\hline \multirow{2}{*}{ Rnd1 } & PlexinA2 & Other & $3 \mathrm{Q} 3 \mathrm{~J}$ & 1279 \\
\hline & PlexinB1 & Other & 2REX & 1342 \\
\hline \multirow[t]{2}{*}{ Rholp } & $\operatorname{Sec} 3 p$ & PH domain & $3 \mathrm{~A} 58$ & 1447 \\
\hline & & Arf family & & \\
\hline \multirow[t]{4}{*}{ Arf1 } & GGA1 & Helical pair (C) & $1 \mathrm{~J} 2 \mathrm{~J}$ & 1208 \\
\hline & AP1 & Helical pair (F) & 4HMY & 1622 \\
\hline & ARHGAP21 & Other $(\mathrm{PH})$ & 2J59 & 1610 \\
\hline & $\mathrm{COP}$ & Helical pair (F) & $3 \mathrm{TJZ}$ & 1745 \\
\hline \multirow[t]{4}{*}{ Arf6 } & JIP4 & Helical pair (F) & $2 \mathrm{~W} 83$ & C: 766 D: 892 \\
\hline & MKLP1 & Intermolecular $-\beta$ & $3 \mathrm{VHX}$ & 2249 \\
\hline & GRP1 Cytohesin & PH domain & $4 \mathrm{KAX}$ & 2555 \\
\hline & CTA1 & Other & 2A5D & 1915 \\
\hline \multirow[t]{2}{*}{ Arl1 } & GRIP & Helical pair (D) & 1UPT 1R4A & 1296 \\
\hline & Arfaptin & Helical pair (E) & $4 \mathrm{DCN}$ & 1484 \\
\hline \multirow[t]{3}{*}{ Arl2 } & UNC119 & Intermolecular $-\beta$ & $4 \mathrm{GOK}$ & 1392 \\
\hline & PDE & Intermolecular $-\beta$ & $1 \mathrm{KSG}$ & 1710 \\
\hline & BART & Other & $3 \mathrm{DOE}$ & 2209 \\
\hline Arl3 & UNC119 & Intermolecular $-\beta$ & 4GOJ & 1867 \\
\hline \multicolumn{4}{|c|}{ Rab family } & \\
\hline Rab3A & Exophilin1/Rabphilin & Helical pair (D) & 1ZBD & 2853 \\
\hline Rab4A & Rabenosyn5 & Helical pair (D) & $1 \mathrm{ZOK}$ & 1788 \\
\hline
\end{tabular}




\begin{tabular}{|c|c|c|c|c|}
\hline Rab5A & $\begin{array}{l}\text { Rabaptin5 } \\
\text { EEA1 }\end{array}$ & $\begin{array}{l}\text { Helical pair }(\mathrm{F}) \\
\text { Other }\end{array}$ & $\begin{array}{l}1 \mathrm{TU} 3 \\
3 \mathrm{MJH}\end{array}$ & $\begin{array}{l}\text { F: } 948 \text { G: } 507 \\
1120\end{array}$ \\
\hline Rab6 & $\begin{array}{l}\text { GCC185 } \\
\text { R6IP1 }\end{array}$ & $\begin{array}{l}\text { Helical pair }(G) \\
\text { Helical pair }(G)\end{array}$ & $\begin{array}{l}3 \mathrm{BBP} \\
3 \mathrm{CWZ}\end{array}$ & $\begin{array}{l}\text { D: } 548 \text { E: } 802 \\
1499\end{array}$ \\
\hline Rab7 & RILP & Helical pair (D) & $1 \mathrm{YHN}$ & 1303 \\
\hline Rab8A & OCRL & Other & 3QBT & 1910 \\
\hline Rab11 & $\begin{array}{l}\text { FIP2 } \\
\text { FIP3 } \\
\text { Myo5b }\end{array}$ & $\begin{array}{l}\text { Helical pair (F) } \\
\text { Helical pair (F) } \\
\text { Helical pair (C) }\end{array}$ & $\begin{array}{l}\text { 2GZD 4C4P } \\
\text { 2D7C } \\
\text { 2HV8 } \\
4 \mathrm{LX} 0\end{array}$ & $\begin{array}{l}\text { C: } 1271 \text { D: } 294 \\
\text { C: } 1352 \text { D: } 300 \\
1959\end{array}$ \\
\hline Rab22 & Rabenosyn5 & Helical pair (D) & $1 \mathrm{ZOJ}$ & 1341 \\
\hline Rab25 & FIP2 & Helical pair (D) & $3 \mathrm{TSO}$ & C: 412 D: 1298 \\
\hline Rab27A & Exophilin4/Sip2A & Helical pair (D) & $3 \mathrm{BC} 1$ & B: 1970 F: 904 \\
\hline Rab27B & Melanophilin/Slac2a & Helical pair (D) & 2ZET & 3720 \\
\hline Rab32 & VARP & Helical pair $(\mathrm{F})$ & $4 \mathrm{CYM}$ & 810 \\
\hline \multicolumn{5}{|c|}{ Ran family } \\
\hline Ran & RanBP2 & PH domain & 1RRP & 4699 \\
\hline & Importin & Other & 2BKU 1IBR 1QBK & 4088 \\
\hline & Exportin & $\begin{array}{l}\text { Other } \\
+ \text { miRNA } \\
+ \text { tRNA } \\
\end{array}$ & $\begin{array}{l}1 \text { WA5 } \\
\text { 3A6P } \\
\text { 3ICQ } \\
\end{array}$ & $3555 ;$ cargo 701 \\
\hline
\end{tabular}

